Portland State University

PDXScholar

6-17-2020

\title{
Design of a 7-MHz Portable Direct Conversion Transceiver with Digitally Controlled Keying
}

\author{
Abram Morphew \\ Portland State University
}

Follow this and additional works at: https://pdxscholar.library.pdx.edu/open_access_etds

Part of the Electrical and Computer Engineering Commons Let us know how access to this document benefits you.

\section{Recommended Citation}

Morphew, Abram, "Design of a 7-MHz Portable Direct Conversion Transceiver with Digitally Controlled Keying" (2020). Dissertations and Theses. Paper 5548.

https://doi.org/10.15760/etd.7422

This Thesis is brought to you for free and open access. It has been accepted for inclusion in Dissertations and Theses by an authorized administrator of PDXScholar. Please contact us if we can make this document more accessible: pdxscholar@pdx.edu. 
Design of a 7-MHz Portable Direct Conversion Transceiver with Digitally Controlled Keying

by

Abram Morphew

A thesis submitted in partial fulfillment of the requirements for the degree of

\author{
Master of Science \\ in \\ Electrical and Computer Engineering
}

Thesis Committee:

Robert Bass, Chair

Branimir Pejcinovic

Erik Sanchez

Portland State University

2020 


\title{
Portland State University
}

\author{
Abstract \\ Department of Electrical and Computer Engineering \\ Maseeh College of Engineering and Computer Science \\ Master of Science
}

by Abram Morphew

\begin{abstract}
This thesis outlines the design of a portable direct conversion transceiver system for the $7-\mathrm{MHz}$ (or $40 \mathrm{~m}$ ) band. This band is popular due to its propagation characteristics, which allow for world-wide communication with very low power. The transceiver utilizes a crystal-stabilized local oscillator optimized for frequency agility, low power consumption, and an optimal drive level of $+7 \mathrm{dBm}$. A low power 8-bit microcontroller acts as an interface for either a straight key providing manual Morse code operation or digital logic control from a personal computer. It also acts as a sidetone oscillator providing audio feedback to the operator during keying and reducing circuit complexity. Switching field-effect transistors (FETs) were used to change from transmit to receive with a switching speed of less than 300 microseconds and allowing for full break-in functionality. For the transmitter portion of the design, a dual-stage power amplifier was developed capable of power output levels greater than $30 \mathrm{dBm}$. Transmission tests were received at several locations ranging from Calgary, Canada to Tucson, Arizona having a maximum propagation distance of 1103.5 miles from the transmitter source.
\end{abstract}


For Bethany, Gracie, and Isabella... 
I'd certainly like to acknowledge the contribution by my thesis advisor, Dr. Richard Campbell, for continually fueling my already existing interest in RF applications. Much appreciation also goes to the thesis committee members, Dr. Robert Bass, Dr. Erik Sanchez, and Dr. Branimir Pejcinovic for their feedback and contribution to my work.

I'd also like to thank the countless amateur operators who have provided access to their software-defined radios over the internet and the development team of the Reverse Beacon Network. Using their tools has allowed for over-the-air testing in a way that wouldn't have been possible otherwise.

Lastly, I'd like to thank my partner, Bethany, for her constant and continued support in all aspects of life. Without her, this work would never have been possible. 
Table of Contents

$\begin{array}{ll}\text { Abstract } & \text { i }\end{array}$

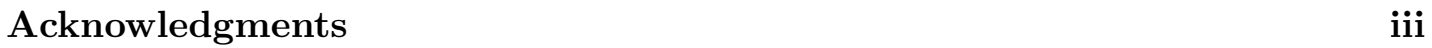

List of Figures $\quad$ vi

List of Tables

Abbreviations $\quad$ ix

1 Introduction $\quad 1$

1.1 Direct Conversion Receiver .................... 1

1.2 Motivation ...................... 4

1.3 Research Objectives ................... 5

1.4 Organization ..................... . . 6

2 Direct Conversion Receiver $\quad \mathbf{7}$

2.1 Receiver Functionality .................... 7

2.2 Diode Ring Mixer . . . . . . . . . . . . . . . . . . . . . 8

2.3 Local Oscillator . . . . . . . . . . . . . . . . . . . . . . 9

2.4 Low-Pass Filter $(\mathrm{LPF})$. . . . . . . . . . . . . . . . . . . . 16

2.5 Low-Noise Amplifier (LNA) . . . . . . . . . . . . . . . . 18

2.6 Audio Amplifier . . . . . . . . . . . . . . . . . . . . 21

3 Transmitter and Switching Circuitry 24

3.1 Transmitter Functionality . . . . . . . . . . . . . . . . . . 24

3.2 Driver Stage . . . . . . . . . . . . . . . . . . . 25

3.3 Final Stage . . . . . . . . . . . . . . . . . . . 28

3.4 Digital Transmit/Receive Switching . . . . . . . . . . . . . . . . . 31

4 Receiver Module Characterization $\quad 34$

4.1 Local Oscillator . . . . . . . . . . . . . . . . . . . . 34

4.2 Low-Noise Amplifier (LNA) . . . . . . . . . . . . . . . . . 38

4.3 Audio Amplifier. . . . . . . . . . . . . . . . . . . . 41

5 Transmitter and Switching Module Characterization 45

5.1 Power Amplifier Characterization . . . . . . . . . . . . . . . 45 
$5.2 \quad$ RF Switch Measurements . . . . . . . . . . . . . . . . . 48

5.3 Digital Control . . . . . . . . . . . . . . . . . . . . 49

$5.4 \mathrm{MCU}$ as a Side-tone Oscillator . . . . . . . . . . . . . . 50

6 Results and Discussion $\quad \mathbf{5 2}$

6.1 Schematic and Board Layout . . . . . . . . . . . . . . . . . . . 52

6.2 Board-level Testing . . . . . . . . . . . . . . . . . . 52

6.3 On-Air Test Results . . . . . . . . . . . . . . . . . . . 55

6.4 Future Work . . . . . . . . . . . . . . . . . . 57

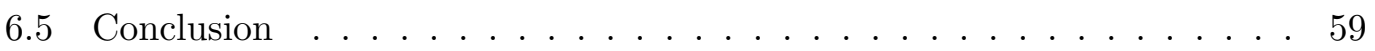

$\begin{array}{lr}\text { A AVR Controller Program } & \mathbf{6 0}\end{array}$

A.1 Keying Control Code for AVR Microcontroller . . . . . . . . . . . . 60

B Bill of Materials $\quad \mathbf{6 6}$

B.1 List of Materials Used in the Final Transceiver . . . . . . . . . . . 66

$\begin{array}{lr}\text { Bibliography } & 68\end{array}$ 


\section{List of Figures}

1.1 Block Diagram of a Direct Conversion Receiver . . . . . . . . . . . . 2

1.2 Frequency Offset in a Direct Conversion Receiver . . . . . . . . . . . . . 3

1.3 Block Diagram of the Direct Conversion Transceiver Design . . . . . . . 5

2.1 Receiver Section of the Direct Conversion Transceiver Block Diagram . 7

2.2 Commutation in a Diode Ring Mixer at the LO Frequency . . . . . . . 8

2.3 Circuit Model of an Ideal Crystal Resonator . . . . . . . . . . . . . . . . 10

2.4 Basic Colpitts-Clapp Oscillator . . . . . . . . . . . . . . . . 11

2.5 Basic Colpitts-Clapp Oscillator with Crystal Resonator . . . . . . . . 13

2.6 Voltage-Controlled Colpitts-Clapp Oscillator with Parallel Resonators . 15

2.7 5th Order Butterworth Low-Pass Filter Designed for $7 \mathrm{MHz}$. . . . . . . 17

2.8 Simplified Single-Tuned Amplifier Schematic . . . . . . . . . . . . . . . 18

2.9 RLC Bandpass Filter Circuit . . . . . . . . . . . . . . . . . . . 19

2.10 Simplified Dual-Stage Audio Amplifier Schematic . . . . . . . . . . . . 21

3.1 Transmitter Section of the Direct Conversion Transceiver Block Diagram 25

3.2 Simulated I-V Characteristics for a BS170 MOSFET . . . . . . . . . . . 26

3.3 Simplified Schematic for a Class A Driver Stage . . . . . . . . . . . . 28

3.4 Plot of Theoretical Amplifier Efficiency . . . . . . . . . . . . 30

3.5 Simplified Schematic of the IRF510 Final Stage Power Amplifier . . . . 31

3.6 Schematic for RF Switch Using a Common-gate JFET Amplifier . . . . 32

3.7 Pinout for the ATTiny85 8-bit Microcontroller . . . . . . . . . . 33

4.1 LTSpice Schematic for the Local Oscillator . . . . . . . . . . . . . . . . . 35

4.2 Simulated FFT of Local Oscillator Output . . . . . . . . . . . . . . . . . . . . . . . . . 36

4.3 Build of Local Oscillator Module . . . . . . . . . . . . . . . . . . 37

4.4 Measured Power Output of Local Oscillator Build . . . . . . . . . . . 37

4.5 Measured Harmonic Content of Local Oscillator . . . . . . . . . . . . . . 38

4.6 LTSpice Schematic for the Single-tuned Low-noise Amplifier . . . . . . . 39

4.7 Build of Low-noise Amplifier Module . . . . . . . . . . . . . . . . . . . 40

4.8 Simulated S-parameters for Single-tuned LNA . . . . . . . . . . . . . . . 40

4.9 Measured S-parameters for Single-tuned LNA . . . . . . . . . . . . . 41

4.10 LTSpice Schematic for the Receiver Audio Stage . . . . . . . . . . . . . 42

4.11 Audio Output Capture at $1 \mathrm{kHz}$ into a $32 \Omega$ Load . . . . . . . . . . . . . . . 42

4.12 Simulated and Measured Amplifier Frequency Response . . . . . . . . . 43

5.1 LTSpice Schematic of the Power Amplifier Design . . . . . . . . . . . . 46

5.2 Simulated and Measured Comparison of Power Amplifier Output . . . . 46

5.3 Measured Spectral Content of the Power Amplifier Output . . . . . . . 47 
5.4 Measured Switching Time of JFET Transistor Switch . . . . . . . . . 48

5.5 Schematic of MCU Transceiver Application Circuit . . . . . . . . . . . . 49

6.1 Full Transceiver Schematic . . . . . . . . . . . . . . . . . . . 53

6.2 Full Transceiver Circuit Board Layout . . . . . . . . . . . . . . . . . . 54

6.3 Transmitter Signal Received on a Software-Defined Radio . . . . . . . . 55

6.4 Stations Reporting Received Transmissions on the Reverse Beacon Net-

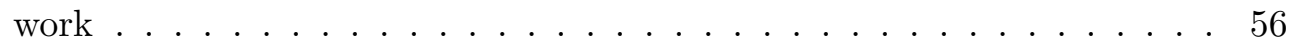

6.5 Logarithmic Audio Spectrograph with and without Multi-stage Filtering 57

6.6 Assembled PCB Mounted in the Aluminum Enclosure . . . . . . . . . . 58

6.7 Completed Transceiver and On-air Test Station . . . . . . . . . . . . . 59 


\section{List of Tables}

2.1 Normalized Values for Butterworth Low-Pass Filters . . . . . . . . . 17

4.1 Simulated/Measured Comparison Table for Local Oscillator . . . . . . . 36

B.1 Bill of Materials . . . . . . . . . . . . . . . . . 67 
Abbreviations

DC Direct Current

DCR Direct Conversion Receiver

DCT Direct Conversion Transceiver

DDS Direct Digital Synthesizer

HF High Frequency

IC Integrated Circuit

IF Intermediate Frequency

LPF Low Pass Filter

LSB Lower Side Band

LO Local Oscillator

PA Power Amplifier

RF Radio Frequency

RIT RX Incremental Tuning

RX Receive

TX Transmit

USB Upper Side Band

XIT TX Incremental Tuning 


\section{Chapter 1}

\section{Introduction}

\subsection{Direct Conversion Receiver}

Since the mid-1960s, a common activity amongst amateur radio operators is to homebrew radio equipment capable of portable use. The desire for small, portable radios to use while mobile or hiking saw renewed interest in the direct conversion receiver due to its lack of multiple intermediate frequency (IF) stages and inherent simplicity. Though originally developed in the 1930s, the first recorded application of direct conversion as a technique is owed to the electronic musical instrument known as the Theremin [1]. By the time this technique was adopted for the purpose of communications, the direct conversion receiver was practically abandoned in favor of other design techniques until around 1961 [2]. The appeal to most amateurs at the time was that a solid-state receiver could be constructed using a minimum number of parts with basic construction techniques and be small enough to slip into a pocket or backpack. While the simplicity of this topology makes it attractive, there are a number of advantages and disadvantages to direct conversion that have to be overcome during the design process.

The block diagram shown in Figure 1.1 gives the outline for a direct conversion receiver (DCR). At the heart of the DCR is a balanced product mixer. The mixer generates 


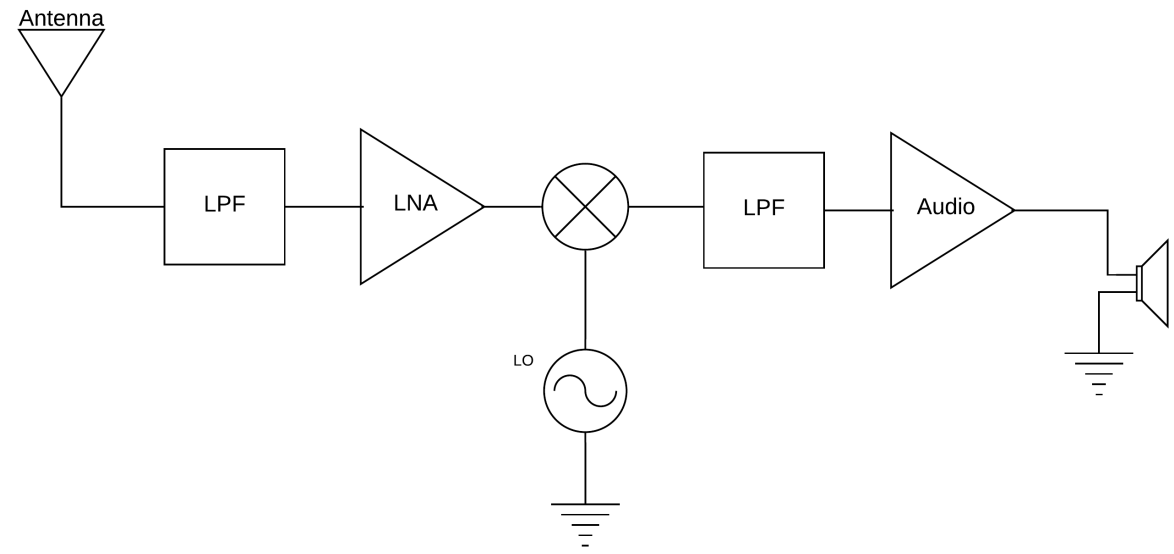

Figure 1.1: Block Diagram of a Direct Conversion Receiver

the product of the incoming RF signal with the local oscillator (LO). The product of two sinusoids can be broken down into the sum and difference of the two signals by the following trigomometric identity [3].

$$
\cos \left(\omega_{1} t\right) \cos \left(\omega_{2} t\right)=1 / 2\left[\cos \left(\omega_{1} t+\omega_{2} t\right)+\cos \left(\omega_{1} t-\omega_{2} t\right)\right]
$$

From this identity, the output of the product mixer includes both the sum and difference of the two input frequencies. Using the direct conversion technique, the frequency of the $\mathrm{LO}$ and incoming RF signal beat together to produce an intermediate frequency (IF) in the audio range. The additional IF signal that is around twice the frequency of the LO is discarded via a low-pass filter at the input to an audio amplifier. This process is sometimes referred to as downconversion whereby the frequency of an RF signal is converted to a lower frequency. Converting the incoming signal directly into the audio range makes for a highly selective receiver front-end. This is in contrast to superheterodyning, which converts the received signal to an intermediate frequency for further processing. Direct conversion is sometimes referred to as zero-IF or homodyne receiving due to its lack of an IF stage. 


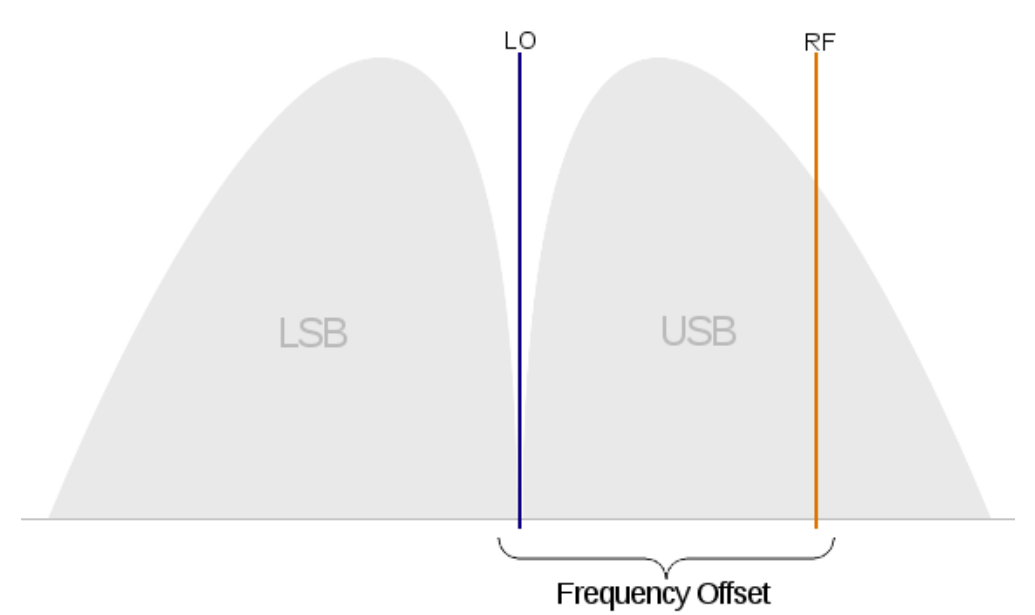

Figure 1.2: Frequency Offset in a Direct Conversion Receiver

The lack of additional IF stages and image rejection filters can also make direct conversion systems tricky to use. Figure 1.2 shows the upper and lower side bands (USB and LSB respectively) surrounding the LO frequency that would be generated from downconversion process. An incoming RF signal with a frequency offset of $700 \mathrm{~Hz}$ above the $\mathrm{LO}$ would produce a $700 \mathrm{~Hz}$ tone in the audio range. Changing the frequency of the LO to match that of the incoming signal would produce a $0 \mathrm{~Hz}$ tone or silence. This is known as zero-beating and is often used to match the frequency of a transmitting signal so that both parties are transmitting on the same frequency. The problem here is that two stations transmitting on the same frequency can't hear each other in the direct conversion system. Therefore, automatic switching between the transmit and receive frequencies is usually desired in order for both stations to use the same frequency to exchange information. Additionally, filtering of one of the side bands is also a common feature of modern commercial receivers in order to prevent the incoming signal from appearing twice as the LO changes frequency. 


\subsection{Motivation}

There are many amateur radio operators that pursue portable operation with homebuilt transceivers. A state-of-the-art transceiver loaded with features like the Elecraft KX3 can be used as a portable transceiver and even comes in a kit form. However, a base package sold as a kit has a base price of $\$ 1149$ USD and might be a bit steep for someone looking to experiment on the high-frequency (HF) bands. Cheaper single band kits are available for sale from overseas distributors for under $\$ 20$ USD, but these kits often have either a lack of features or such poor performance that they are hardly usable when it comes to contacting other people on the air. The Elecraft KX1 was a 4-band CW transceiver that was originally sold around \$400 USD, which offered good performance, a sturdy package, and a variety of features making it ideal for portable use in the field. However, the KX1 was discontinued in 2017 due to component availability issues, further increasing the price gap in low-power HF transceivers [4]. QRP Labs in the United Kingdom has taken to filling this price gap with their 5-watt QCX mono-band transceiver for $\$ 50$ USD on a single printed circuit board without any form of enclosure [5]. Due to its use of a direct digital synthesizer (DDS) and other digital components, the QCX pulls more than $120 \mathrm{~mA}$ during receive. While these middle-of-the-road designs are making a lot of progress in the realm of cost, better power management is desired for long-duration portable use. The primary motivation behind this research is to develop a single-band transceiver that achieves power efficiency and the performance similar to analog direct conversion designs of the past while incorporating cost-effective and commonly available components. This will advance the state-of-the-art by determining if analog design blocks can be controlled by a low-cost and power-efficient microcontroller. A block diagram for this type of design can be seen in Figure 1.3. 


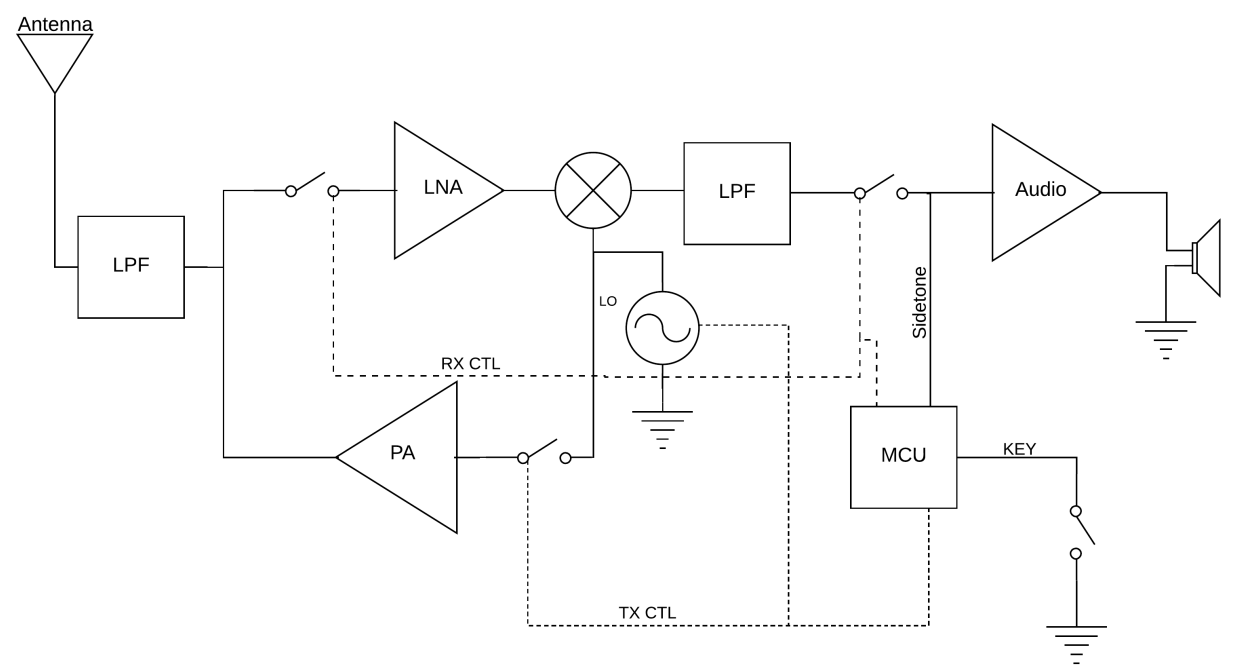

Figure 1.3: Block Diagram of the Direct Conversion Transceiver Design

Referring to Figure 1.3, the diagram can be divided into an upper and lower section compromising the receiver $(\mathrm{Rx})$ and transmitter $(\mathrm{Tx})$, respectively. These two sections must be switched on and off very quickly as the key makes contact with ground. A microcontroller unit (MCU) uses separate control busses to turn off one section before enabling the other. A microcontroller with a clock speed of a few megahertz is necessary in order to make this switching action happen within microseconds. An additional feature of the Tx control bus is to supply a signal to shift the frequency of the LO by a few hundred Hertz. This automatic frequency adjust applies the frequency offset discussed previously and shown in Figure 1.2.

\section{$1.3 \quad$ Research Objectives}

While substantial research has been done previously regarding the direct conversion technique, the overall objective of this paper is to determine if adequate performance can be obtained by using modern low-cost digital components while also reducing circuit complexity. Being a design for portable use, this typically implies that the design should be lightweight and have small footprint. Being limited on size means 
that circuity complexity should be reduced whenever possible in order to accomodate space in the transceiver's enclosure. The overall design criteria for this project are as follows:

- Design a 7-MHz direct conversion transceiver for portable use with a $12 \mathrm{~V}$ battery.

- Transmitter should be capable of power output levels greater than $30 \mathrm{dBm}$.

- Receiver should be able to detect RF power levels down to $-120 \mathrm{dBm}$.

- Utilize a microcontroller for precision controlled transmit/receive switching.

- Determine if adequate RF performance can be obtained using modern digital circuitry.

\subsection{Organization}

This thesis is orgainzed into seven main chapters. The first section (Chapter 1) provides a brief introduction in to the basic principles and history behind the direct conversion receiver along with a modular block diagram describing the entire system design. Chapter 2 outlines the circuit topologies chosen for the receiver section of the design, while Chapter 3 focuses on the transmitter design. Chapters 4 and 5 outline the performance acheived from each module in the receiver and transmitter sections. The results of the whole transeiver system are discussed in Chapter 6 , along with additional work that could be done to improve the system. 


\section{Chapter 2}

\section{Direct Conversion Receiver}

\subsection{Receiver Functionality}

In Figure 2.1, the receiver and transmitter are divided into the upper and lower chains respectively. Both sections share the functionality of the local oscillator thanks to a switch controlled by the MCU. When in the Rx state, the path of RF flows into the system from the antenna, is downconverted to the audio range, and then amplified into a loudspeaker or low-impedance head phone set. The following sections will detail the theory and design decisions made for each individual block of the receiver system.

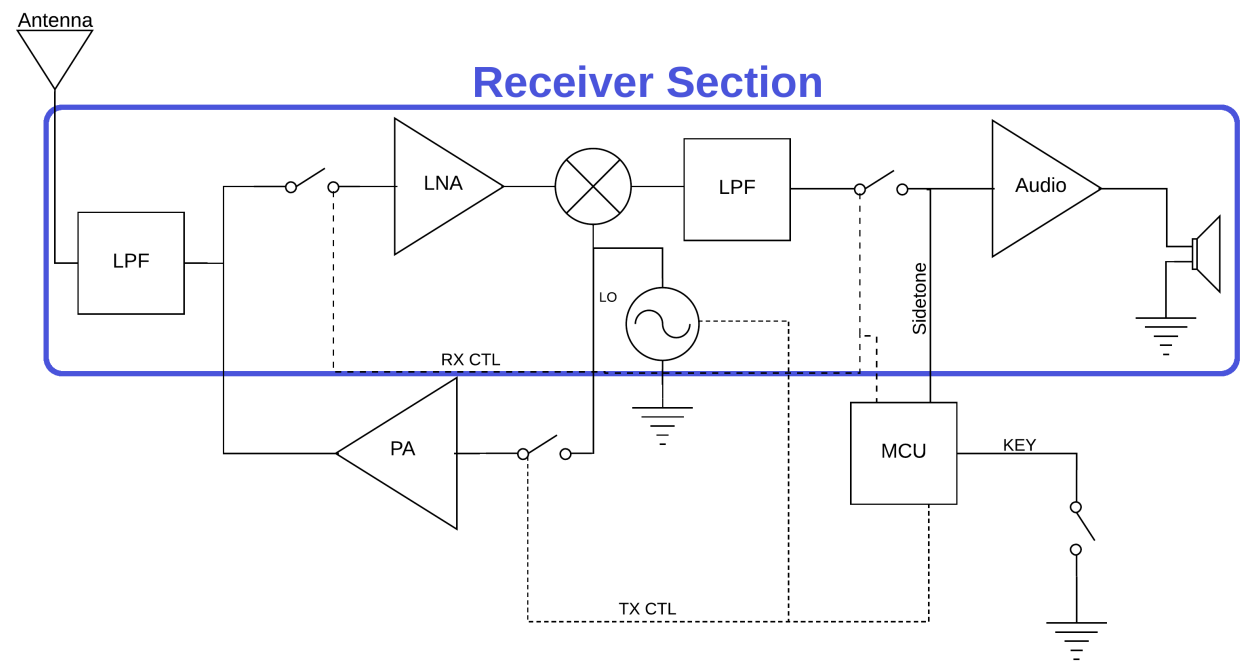

FiguRE 2.1: Receiver Section of the Direct Conversion Transceiver Block Diagram 

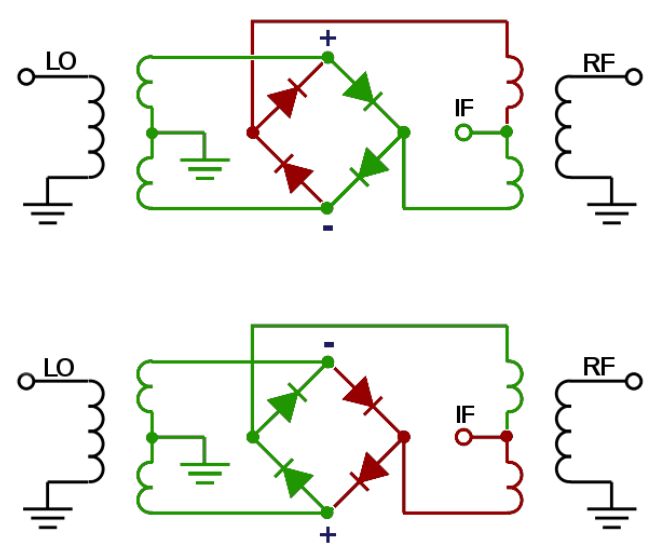

Figure 2.2: Commutation in a Diode Ring Mixer at the LO Frequency

\subsection{Diode Ring Mixer}

The real action of the direct conversion receiver (DCR) is the action of the product mixer downconverting the RF singal into the audio range. This fairly simple trigonometric identity is exactly what allows the end user to "receive" a signal, and the requirements of the product mixer dictate the design requirements for the other modules in the system. Product mixing is a result of two signals applied to any device with square law curvature such as a diode or FET [6]. Therefore, mixers tend to come in two varieties: active and passive. Active mixers can be developed from a single or dual gate FETs and have some advantanges over passive mixers but can suffer from intermodulation (IM) products if not designed with specific considerations [6]. An active mixer in this application would draw additional current and would require a higher number of components if designed from discrete components. Therefore, a Mini-Circuits ADE-1 diode ring mixer was chosen due to its small size and availability.

The ADE- 1 is a class- 1 doubly-balanced mixer with a $+7 \mathrm{dBm}$ LO drive level and an operational frequency range of 0.1 to $500 \mathrm{MHz}$ [7]. A doubly-balanced mixer is ideal as it provides superior dynamic range and with minimal intermodulation (IM) products 
[8]. The electrical schematic for the ADE-1 shows (Figure 2.2) the LO acts on the diodes as switches biasing one pair of diodes and then the other as the polarity of the LO changes. This switching action is known as commutation, and it permits the RF signal to pass through one half of the coupling transformer or the other, effectively multiplying the RF signal by \pm 1 at the LO frequency [9]. The signal present on the IF port is then the sum and difference of the signals present on the LO and RF ports. A wideband termination of $50 \Omega$ at the IF port is useful to ensure that IM products aren't reflected back into the mixer [10].

\subsection{Local Oscillator}

The local oscillator is a crucial element in virtually any radio system. As a result, numerous design techniques have been developed to acheive both frequency stability and selectivity. When choosing an oscillator circuit for this project, several considerations were made. The initial concerns were that the LO needed to be frequency agile across the $\mathrm{CW}$ portion of the $7-\mathrm{MHz}$ band, frequency stable within $\pm 5 \mathrm{ppm}$ for narrow-band tuning, and have a fairly low current draw to extend battery life for portable operation with a $12 \mathrm{~V}$ battery. Additionally, the LO needed to have some voltage control capability in order to apply the frequency offset needed between transmit and receive. Given that the overall design was intended to work within a single band having a tuning range of only $100 \mathrm{kHz}$, the decision was made to employ a variable crystal oscillator (VXO) as the system LO.

Crystal resonators are typically produced from quartz. Quartz has a crystalline atomic structure that can be deformed when acted on by a force. If that force is removed, the quartz structure returns to is previous state almost immediately and acts as a restoring force. When exposed to an electric field, an applied force then acts on the 


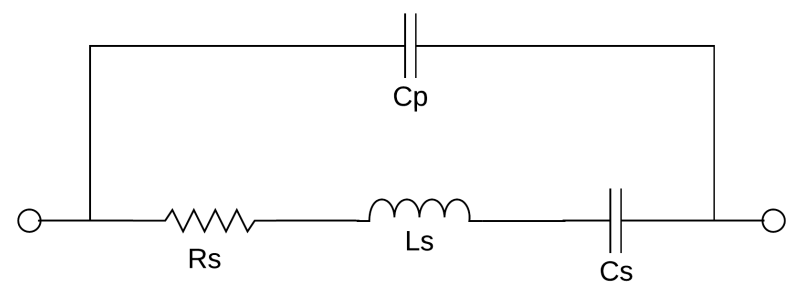

Figure 2.3: Circuit Model of an Ideal Crystal Resonator

electrical charges that are trapped inside the lattice creating a physical distortion in the material known as the piezoelectric effect. Application of an RF signal can cause reflections inside the crystal structure that resonate at specific frequencies realtive to phase shifts created by the crystal's thickness. The piezoelectric property of quartz allows it to be resonant at fundamental as well as overtone frequencies, resulting in little energy loss and a high quality factor (Q). The atomic structure of the crystal also winds up having a very low frequency drift temperature thanks to very small changes that occur as the material expands and contracts [11].

In order to design a crystal osciallator, an electrical circuit aproximation of a quartz crystal is necessary. This idealized model is shown in Figure 2.3. In the model, there are two main paths. The bottom path contains a series RLC circuit for modeling the fundamental resonant frequency of the crystal [12]. The fundamental frequency is usually the frequency labeled on the outer package for ranges of 1 to $30 \mathrm{MHz}$. The series resistance $\left(R_{s}\right)$, inductance $\left(L_{s}\right)$, and capacitance $\left(C_{s}\right)$ parameters describe the motional characteristics related to the mechanical deformation of the crystal while $C_{p}$ represents the parallel capacitance created by the electrodes. Having both series and parallel paths allows the crystal to be resonant at more than one frequency [13]. Overtone frequencies at odd harmonics are also possible but not necessary for the scope of this design. 


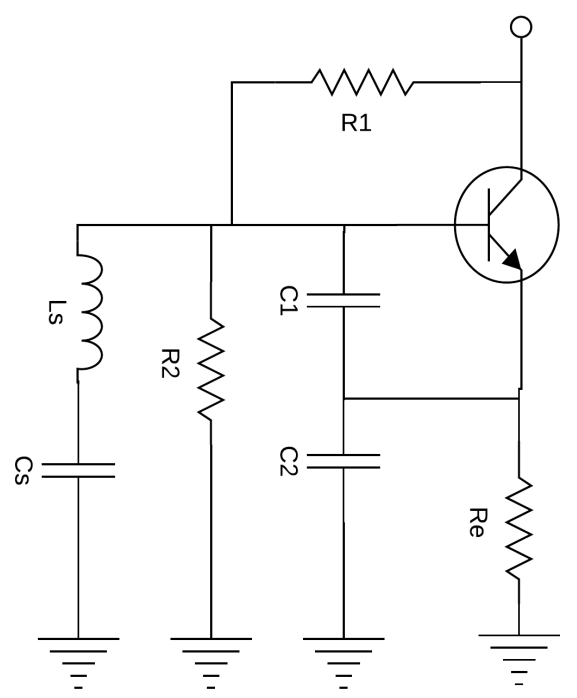

Figure 2.4: Basic Colpitts-Clapp Oscillator

Having such a high motional inductance, it is common practice to see a quartz crystal used in place of an inductor in an LC oscillator. An LC oscillator is a type of feedback oscillator that utilizes resonance of an inductor and capacitor to select the frequency of oscillation. Feedback oscillators are governed by the Barkhausen stability criteria, which require that the gain of the amplifier be greater than or equal to unity and the input signal from the feedback path be in phase with the output [14]. To take advantage of the series inductance that accompanies a quartz crystal, a Colpitts-Clapp oscillator was chosen for its simplistic design.

Figure 2.4 shows a circuit diagram for a simple Colpitts-Clapp oscillator. Looking at the circuit from the point of $\mathrm{AC}$ analysis, there are four main reactive components. Being an LC oscillator, resonance is achieved by a tank circuit whose frequency selectivity is expressed by the following relationship.

$$
f_{0}=\frac{1}{2 \pi \sqrt{L_{s} C_{e q}}}
$$


Equation 2.1 gives the expression for the resonant frequency of the tank circuit, $f_{0}$, as a function of the inductor, $L_{s}$, and the equivalent capacitance, $C_{e q}$. Combining all the capacitors in series results in Equation 2.2.

$$
C_{e q}=\left(\frac{1}{C_{s}}+\frac{1}{C_{1}}+\frac{1}{C_{2}}\right)^{-1}
$$

From the point of $\mathrm{AC}$ analysis, $C_{1}$ and $C_{2}$ create a reactive voltage divider at the emitter of the transistor. The ratio of the two capacitors control the amount of positive feedback being directed back into the amplifier. This is convenient as the closed loop gain of the amplifier can then be expressed by equation 2.3 .

$$
A_{v}=\frac{X_{C 2}}{X_{C 1}}
$$

The fundamental frequency of the crystal resonator is set by the series inductance and capacitance values given by the model shown in Figure 2.3. The crystal model then can be used to replace the series LC tank circuit comprised of $L_{s}$ and $C_{s}$ in Figure 2.4. Being a drop-in replacement for the LC tank, it becomes clear as to why this circuit topology is then a typical choice for a crystal oscillator. The values of $L_{s}$ and $C_{s}$ can be substituted by the motional inductance and capacitance in the crystal. Since the motional inductance is very large (likely in $\mathrm{mH}$ ) and motional capacitance very small (likely in $\mathrm{fF}$ ), external components can be used to alter the resonant frequency of the circuit, "pulling" the resonant frequency away from the intrinsic frequency of the crystal [15]. The substitution of the crystal model and addition of external tuning elements can be seen in Figure 2.5. 


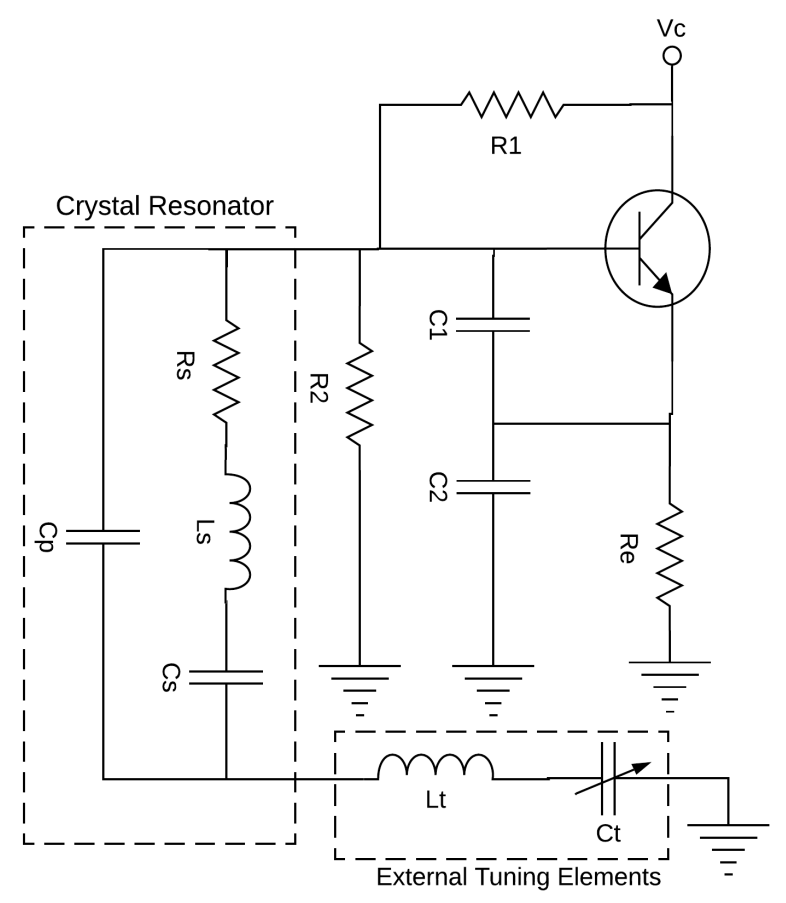

Figure 2.5: Basic Colpitts-Clapp Oscillator with Crystal Resonator and External Tuning Elements

The pulling range of the crystal-controlled oscillator with the variable capacitor $\left(C_{t}\right)$ is likely limited by the extremely small capacitance of $C_{s}$ inside the resonator. Being on the order of femtofarads, the $C_{s}$ term dominates the total equivalent capacitance in the circuit. Additionally, the inductor external to the crystal resonator $\left(L_{t}\right)$ would need to to be a very large, high $\mathrm{Q}$ inductor in order to be able compete with the motional inductuance of the crystal. By placing one or more additional crystals in parallel at the cost of sacrificing space on the circuit board, the motional inductance is decreased while the motional capacitance is increased [16]. This should make the external tuning elements more effective at pulling the frequency and extending the pulling range. The value of $L_{t}$ and $C_{t}$ will likely need to be determined either experimentally or by component availability since the motional inductance and capacitance in the crystal is unknown. 
With a suitable plan of attack developing a tuning range across the band of interest, a voltage control method was still needed in order to allow the MCU to switch between transmit and receive frequencies. A method for acheiving this goal would be to place a voltage-controlled capacitor (also known as a varactor) in parallel with the tuning capacitor, $C_{t}$. The small signal junction capacitance $\left(C_{j}\right)$ for a diode is given in Equation 2.4.

$$
C_{j}=\frac{C_{j 0}}{\sqrt{\psi_{0}+V_{R}}}
$$

In this equation, $C_{j 0}$ represents the intrinsic junction capacitance, $\psi_{0}$ is the built-in potential, and $V_{R}$ is the reverse bias voltage [17]. One method of adding voltage-control frequency tuning to this design would be to apply a voltage to a reverse biased Zener diode in parallel with $C_{t}$. The Zener diode in this case acts as a voltage controlled capacitor adding parallel capacitance to the tuning ciruit. This technique has been employed in other direct conversion transceivers using a voltage regulator and potentiometer acting as an incremental tuning system for zero-beating incoming signals [18]. Figure 2.6 shows the addition of the voltage-controlled tuning elements and parallel crystal resonators.

Having developed an outline for a tunable oscillator, there still lies the issue of load impedance and output power. The oscillator needs to be able to drive the ADE-1 ring mixer with a $7 \mathrm{dBm}$ into the $50 \Omega \mathrm{LO}$ port. In keeping with a design optimal for battery usage, the transistor needs to have a DC biasing scheme resulting in around 1 to $3 \mathrm{~mA}$ of current draw. An RF choke (RFC) should also be added to $V_{c}$ in order to prevent RF leakage to power supply. Voltage across an inductor can be expressed in the following form. 


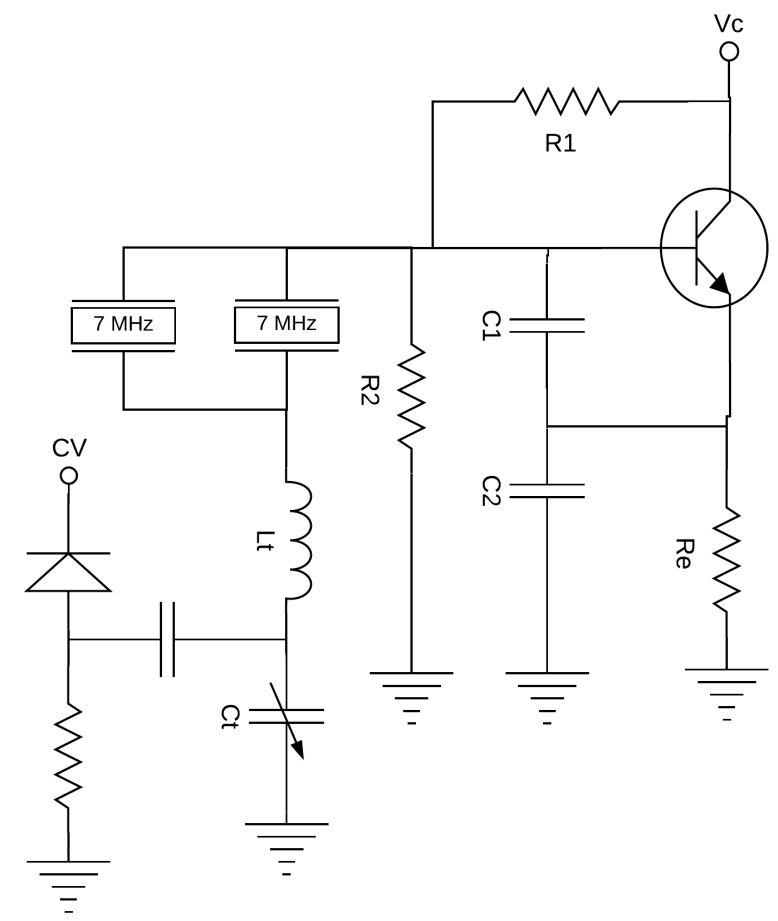

Figure 2.6: Voltage-Controlled Colpitts-Clapp Oscillator with Parallel Resonators

$$
V_{L}=L \frac{d I_{C}}{d t}
$$

Equation 2.5 states that the voltage across an inductor is the product of its inductance times the derivative of the current with respect to time [19]. By placing a large inductor on the order of tens of $\mathrm{mH}$ at $V_{C}$, a high impedance of a few $k \Omega$ is presented at the collector. If $I_{C}$ is modelled as the sum of the DC and AC currents, then there is the potential for $V_{C}$ to exceed the power supply voltage $\left(V_{c c}\right)$, provided a transistor with enough gain is chosen. A general purpose bi-polar junction transistor such as 2N3904 or BC547 having a $\beta$ value in the 100 to 300 range would likely suffice for this purpose.

$$
V_{C}=V_{c c}+V_{L} \sin (\omega t)
$$


This action has the potential for creating a very large voltage at the collect output but with a high impedance. A simple technique for dropping this impedance is to capacitively couple the output from the collector to an emitter-follower stage. Emitter resistance for an NPN transistor can be calculated by equation 2.7 [21].

$$
r_{e}=\frac{0.026}{I_{E}}
$$

By taking the output of an emitter-follower buffer stage, a modest current around 2 $\mathrm{mA}$ or less should provide an output impedance around $50 \Omega$ or lower. This should be suitable for reaching the drive levels needed by the product mixer.

\subsection{Low-Pass Filter (LPF)}

Looking back at Figure 2.1, the receiver design calls for two low-pass filters. The first is an RF filter before the LNA stage intended to attenuate out-of-band signals above the cutoff frequency and attenuate additional harmonics from the transmitter. The second is an audio frequency (AF) filter intended to attenuate the $14 \mathrm{MHz} R F$ signal coming out of the mixer. The RF filter was designed first to have a characteristic impedance of $50 \Omega$ starting with equations 2.8 and 2.9 [9]. A table of normalized filter values is shown in Table 2.1.

$$
\begin{aligned}
& L(n)=\frac{g(n) Z_{0}}{2 \pi f} \\
& C(n)=\frac{g(n)}{2 \pi f Z_{0}}
\end{aligned}
$$




\begin{tabular}{|l|l|l|l|l|l|l|l|}
\hline $\mathbf{N}$ & $\mathbf{g}(\mathbf{1})$ & $\mathbf{g}(\mathbf{2})$ & $\mathbf{g}(\mathbf{3})$ & $\mathbf{g}(\mathbf{4})$ & $\mathbf{g}(\mathbf{5})$ & $\mathbf{g}(\mathbf{6})$ & $\mathbf{g}(\mathbf{7})$ \\
\hline \hline 2 & 1.414 & 1.414 & & & & & \\
\hline 3 & 1.0 & 2.0 & 1.0 & & & & \\
\hline 4 & 0.765 & 1.850 & 1.850 & 0.765 & & & \\
\hline 5 & 0.618 & 1.618 & 2.0 & 1.618 & 0.618 & & \\
\hline 6 & 0.518 & 1.414 & 1.932 & 1.932 & 0.141 & 0.518 & \\
\hline 7 & 0.445 & 1.247 & 1.802 & 2.0 & 1.802 & 1.247 & 0.445 \\
\hline
\end{tabular}

TABLE 2.1: Normalized Values for Butterworth Low-Pass Filters

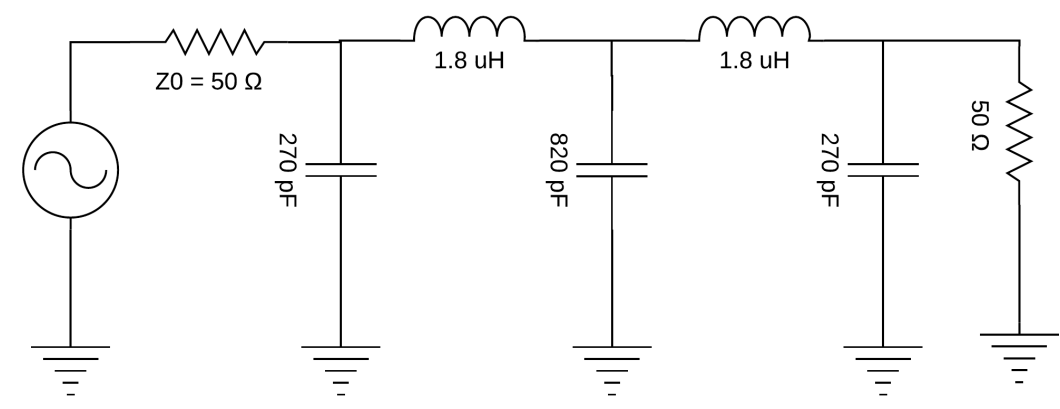

Figure 2.7: 5th Order Butterworth Low-Pass Filter Designed for $7 \mathrm{MHz}$

Using the values in Table 2.1, design of the LPF is fairly trivial providing both loads at the input and output of the filter have a characteristic impedance of $Z_{0}$. Being that the transistors chosen for this design are mostly general purpose, datasheets supplied by the manufacturer don't typically include S-parameter data. Therefore, it's very likely that some impedance matching will need to be done experimentally to ensure optimal performance. Figure 2.7 shows a 5th-order low-pass Butterworth filter design with commonly-available component values.

For the audio filter, the same technique could be used for a third-order low-pass filter at audio frequency. This is useful because the ADE- 1 mixer needs to be properly terminated with a $50 \Omega$ load. A third-order filter should provide around 50 to 60 $\mathrm{dB}$ of attenuation at $10 \mathrm{kHz}$ with some possible ripple in the pass band. Additional filtering may also be achieved by inclusion in the design of the audio amplifier. Some experimentation with component value here may be necessary to find an optimal level 


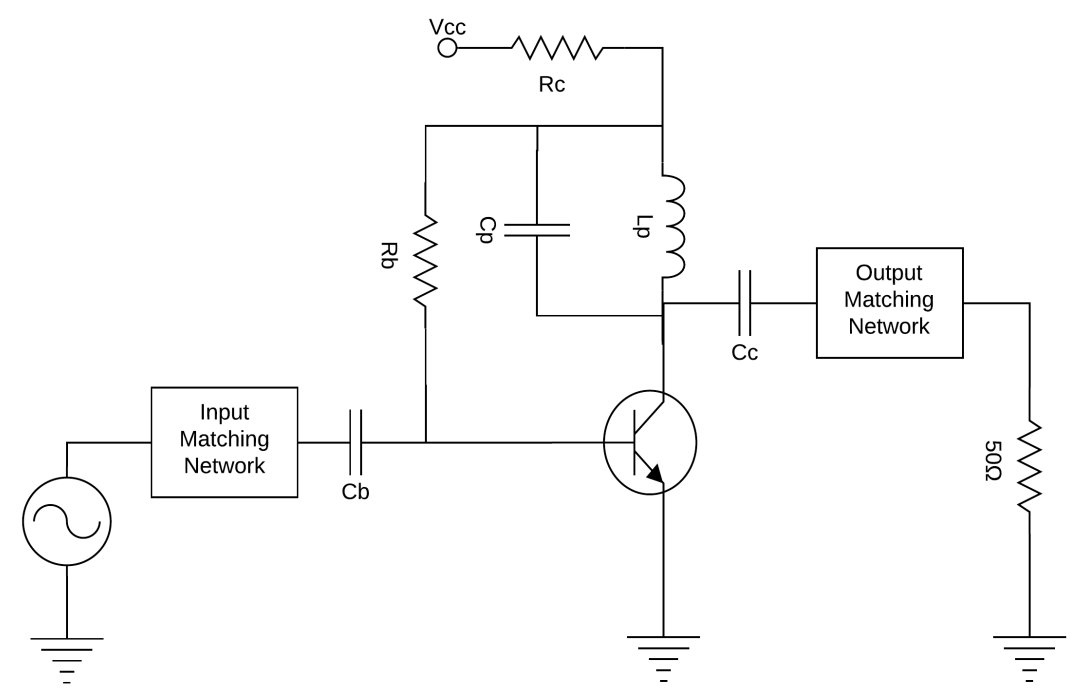

Figure 2.8: Simplified Single-Tuned Amplifier Schematic

of attenuation above $1 \mathrm{kHz}$.

\subsection{Low-Noise Amplifier (LNA)}

The low-pass filter introduced in the previous section is a passive circuit. Undoubtedly, there would be some loss in the the pass band of the filter even with components having a relatively high $\mathrm{Q}$ value. Additionally, some attenuation for frequencies around $1 \mathrm{MHz}$ is desired since this is the range of the AM broadcast band [22]. Signals in this band can be excessively strong and have the potentional to overwhelm the receiver. A simple solution to this would be to use a single-tuned circuit as a low-noise amplifier to ensure weak signal detection over the band of operation.

The single-tuned amplifier has seen use commonly as an IF amplifier in various forms of superheterodyne receivers [23]. Design of this circuit generally begins with a resonant RLC bandpass filter with a given center frequency and bandwidth[24]. Bandwidth considerations can be made by choosing specific bias resistor values, thereby altering the $\mathrm{Q}$ of the filter. Figure 2.8 shows a simplified schematic of this circuit topology. 


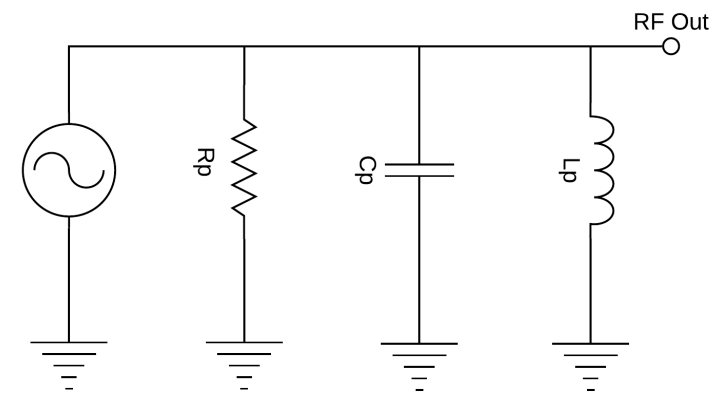

Figure 2.9: RLC Bandpass Filter Circuit

From the point of an AC analysis looking at the node of the collector of the transistor, the ratio of the voltage here to the bias current of the transistor appears as a resistance $\left(R_{p}\right)$. The schematic can then be simplified into the components of a basic RLC circuit like that shown in Figure 2.9. The transfer function in the s-domain is shown in Equation 2.10.

$$
H(s)=\frac{1}{R C} \frac{s}{s^{2}+\frac{s}{R C}+\frac{1}{L C}}
$$

Analysis into the frequency domain produces the separate equations that are key parameterizations of a typical bandpass filter. There are the expressions of resonant frequency $\left(f_{0}\right)$, bandwidth $(B W)$, and quality factor $(Q)$, which are given in Equations 2.11, 2.12, and 2.13 [19]. A bandpass filter has two cutoff frequencies at the band edges where the attenuation drops to $3 \mathrm{~dB}$. These frequencies are the upper $\left(f_{U}\right)$ and lower $\left(f_{L}\right)$ cutoff frequencies.

$$
f_{0}=\frac{1}{2 \pi \sqrt{L_{p} C_{p}}}
$$




$$
\begin{gathered}
B W=f_{U}-f_{L}=\frac{1}{2 \pi R_{p} C_{p}} \\
Q=\frac{f_{0}}{f_{U}-f_{L}}
\end{gathered}
$$

By chosing a center frequency and bandwidth, the values of $L_{p}$ and $C_{p}$ can be found by picking a value for one and solving for the other. In this case, a $470 \mathrm{pF}$ capacitor was chosen initially, and the inductor value was calculated as a result. From the vantange point of the collector, $R_{p}$ is the ratio of the collector voltage $\left(V_{c}\right)$ and collector current $\left(I_{c}\right)$. This expression is given below.

$$
R_{p} \approx \frac{V_{c}}{I_{c}} \approx \frac{V_{c}}{\beta I_{b}}
$$

Equation 2.14 then represents a current-controlled resistance by way of the base current of the transistor. Decreasing the base current increases the overall Q of the circuit, making it a variable $\mathrm{Q}$ amplifier. By solving for a particular biasing scheme, the parameters of the filter can now be established. An impedance transformation and isolation of the RF signal from the collector will likely need to be added in order to ensure a good $50 \Omega$ match. Some frequency shift may also occur due to the internal capacitance of the transistor, but this gives a sound method for the foundation of the design work. Design of the input and output matching networks in Figure 2.8 will be determined by the characteristics of the transistor over the operating frequency range and the loading requirements of the product mixer. 


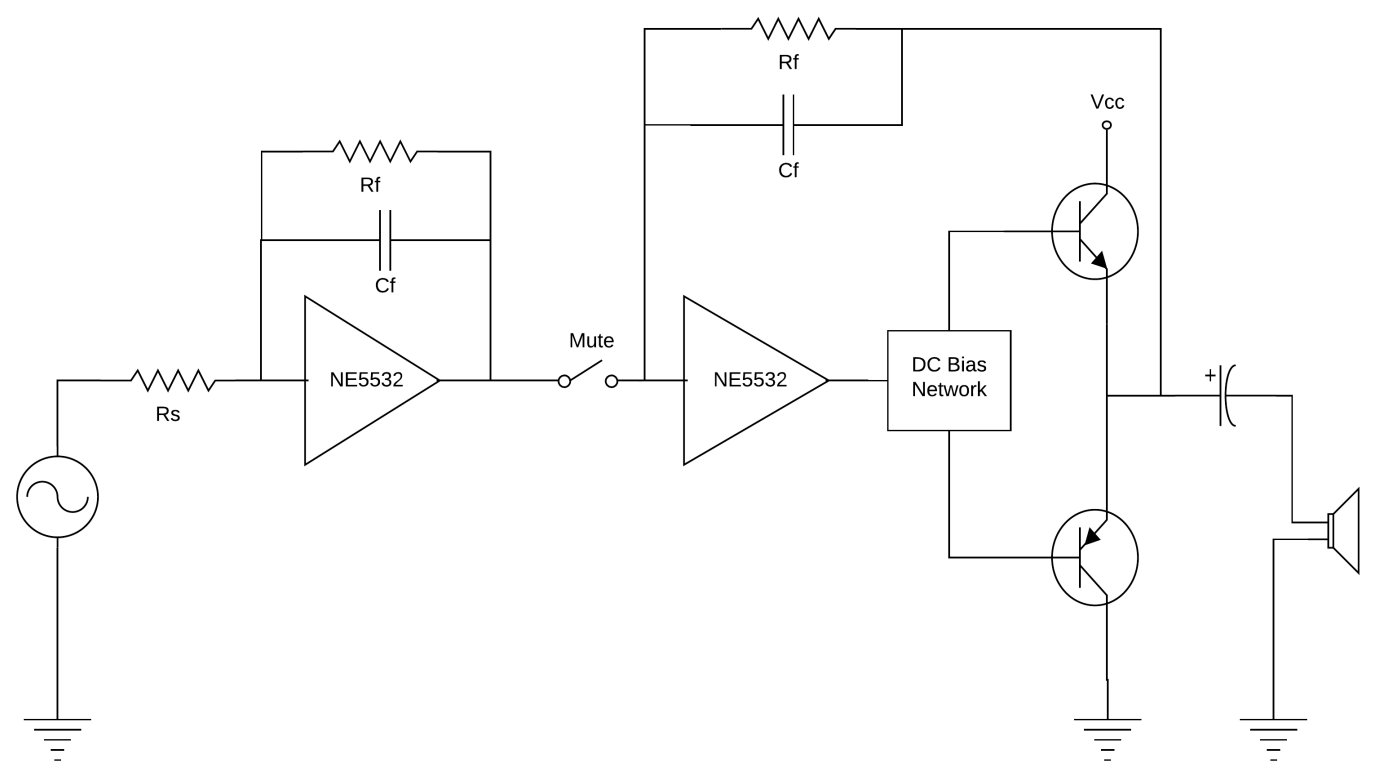

Figure 2.10: Simplified Dual-Stage Audio Amplifier Schematic

\subsection{Audio Amplifier}

The final section of the receiver is the audio amplifier where RF downconverted into the audio spectrum gets delivered to a speaker of some kind. The target load in this case would be somewhere around 300 to $500 \mathrm{~mW}$ into a small $8 \Omega$ speaker. In order to save space, an NE5532 dual operation amplifier was chosen to act as a dual-stage voltage amplifier to drive a class $\mathrm{AB}$ complimentary transistor output stage. A simplified diagram of this technique is shown in Figure 2.10.

The entire audio amplifier schematic can be broken into three make sections from left to right. The first section is comprised of an active first-order low-pass filter using the first half of the NE5532 opamp. Utilizing the inverting input of the opamp, there are two main equations for the design [20].

$$
A_{v}=\frac{-R_{f}}{R_{s}}
$$




$$
f_{c}=\frac{1}{2 \pi R_{f} C_{f}}
$$

The voltage gain $\left(A_{v}\right)$ of the input stage is set by the ratio of $R_{f}$ and $R_{s}$, as shown in equation 2.15. Equation 2.16 sets the cutoff frequency of the low-pass filter. The filter provides additional attenuation in the audio range with a gentle slope of $6 \mathrm{~dB}$ per octave combining with the passive filter between the input of the audio amplifier and the mixer. This was included in the design to provide a way of dialing in an optimal amount of filtering in the event more or less was needed. The output of the active filter is then fed to a voltage-controlled mute switch that allows the MCU to mute the audio when switching between transmit and receive.

The next stage is the second half of the NE5532, which acts as a voltage driver for a class-AB output stage. According to the datasheet for the device, the maximum short-circuit output current is $60 \mathrm{~mA}$, which means that the opamp itself isn't ideal for driving a low-impedance load at the desired power level. It can, however, produce a very high voltage gain into a high-impedance load. Utilizing two BJTs for the output stage with a high $\beta$ presents a high-impedance to the output of the opamp. A feedback network is then taken from the output of the next stage and fed to the input. Using Equations 2.15 and 2.16, this amplfier can also act as another active filter if additional filtering is desired.

The output stage is a class-AB complementary symmetry amplifier stage composed of PNP and NPN BJTs. This acts as a current amplifying device that takes the small current output from the opamp and delivers the current to the loudspeaker or headphones. The class of power amplifier is ultimately determined by its conduction angle and class-AB is really a combination of two separate classes [25]. Class $\mathrm{A}$ is when 
the conduction angle is a full $360^{\circ}$ of the input signal. This means that the transistor is biased on through the entire cycle of a sinusoidal input. Class B by comparison is when the conduction angle is $180^{\circ}$ of the input signal or half of the input signal. By splitting the input signal into two halves, the efficiency of the amplifier can be increased. However, a bias voltage of around $0.6 \mathrm{~V}$ is needed to turn the transistor on. Therefore, Class-B operation produces noticeable distortion products in the audio range known as crossover distortion. Class-AB is the compromise to this where the conduction angle for both transistors is greater than $180^{\circ}$ but less than $360^{\circ}$ [25]. The DC bias network shown in Figure 2.10 sets the bias so that crossover distortion products are eliminated while also keeping efficiency in the $60-70 \%$ range. This technique should provide a very clean audio output keeping distortion products to a minimum and providing less audio distortion over a commercially available IC audio amplifier like the LM386 [26]. 


\section{Chapter 3}

\section{Transmitter and Switching Circuitry}

\subsection{Transmitter Functionality}

In this section, the design specification for the transmitter and switching sections of the transceiver are outlined. This transceiver system is centered around the local oscillator being switched between the transmit and receive circuitry. When the keyer switch is open, the system is in receive mode and the LO is connected directly to the product mixer. For transmit, the LO is connected to the input of the amplifier. The drive level of the $\mathrm{LO}$, however, is design to deliver $+7 \mathrm{dBm}$ or $5 \mathrm{~mW}$ of power. A desired transmit level would be somewhere around $+30 \mathrm{dBm}$ (1 Watt) or greater. In order to acheive at least $23 \mathrm{~dB}$ of gain, a dual-stage power amplifier design will likely be required. The first stage will provide voltage gain using a small signal transistor while the second stage will provide the current gains for deliviering power to a $50 \Omega$ load.

The design techniques and theory used for switching between transmit and receive will also be outlined in this chapter. This will include design of the RF switches themselves as well as discussion of control bus management with the MCU. The MCU has multiple fuctions that tie the system together including the sidetone oscillator, which provides keying feedback to the operator. Being a programmable MCU, a discussion of additional features that are purely based in software is also warranted. 


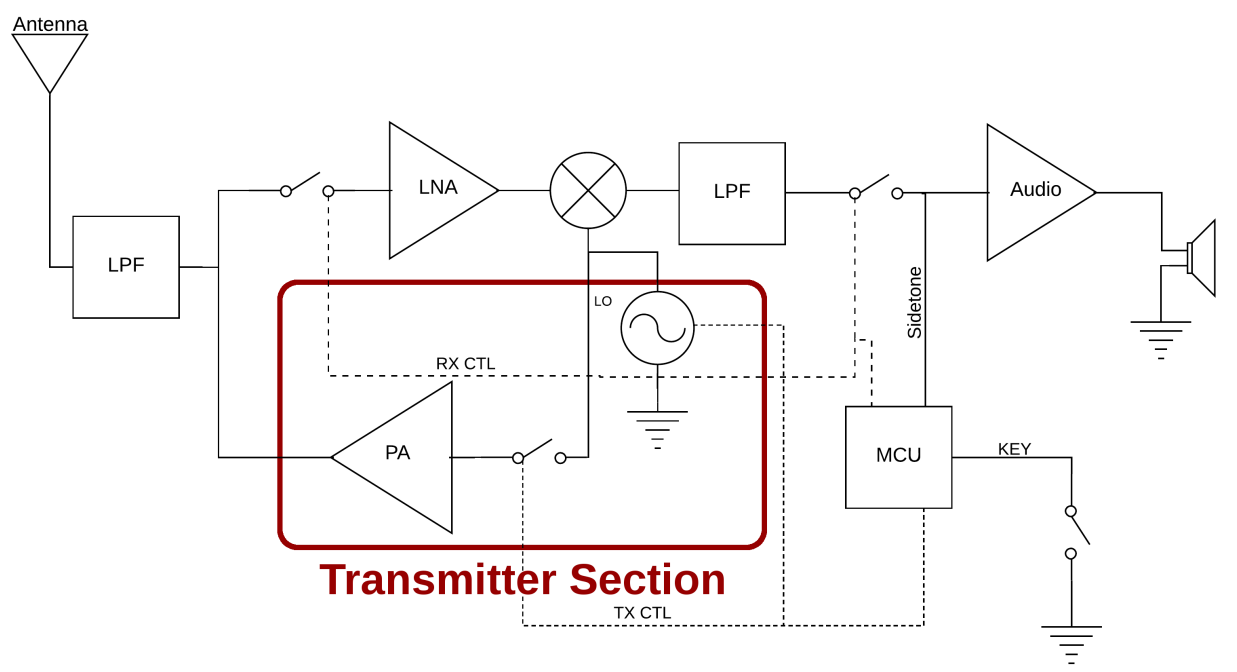

Figure 3.1: Transmitter Section of the Direct Conversion Transceiver Block Diagram

\subsection{Driver Stage}

As mentioned previously, the power amplifier section needs to first contain a small signal voltage driver. Being a portable radio transceiver operated by battery power, some forethought should be given to amplifier effeciency.

$$
\eta=\frac{P_{\text {out }}}{P_{D C}}=\frac{P_{\text {out }}}{P_{\text {out }}+P_{\text {diss }}}
$$

Equation 3.1 is the expression for amplifier efficiency $(\eta)$ where $P_{\text {out }}$ is the RF power output and $P_{D C}$ is the total power drawn from the power supply [27]. The efficiency of a power amplifier is usually associated with its class of operation. A discussion of the audio power amplifier in the previous chapter gives a description of classes A, B, and $\mathrm{AB}$ in terms of conduction angle for the active device delivering power to the load. By exploiting the conduction angle, amplifier efficiency can be increased since the amount of time that the transistor is conducting is reduced. In turn, this decreases the power dissipated $\left(P_{\text {diss }}\right)$. For the design of the voltage driver, a BS170 MOSFET was chosen 


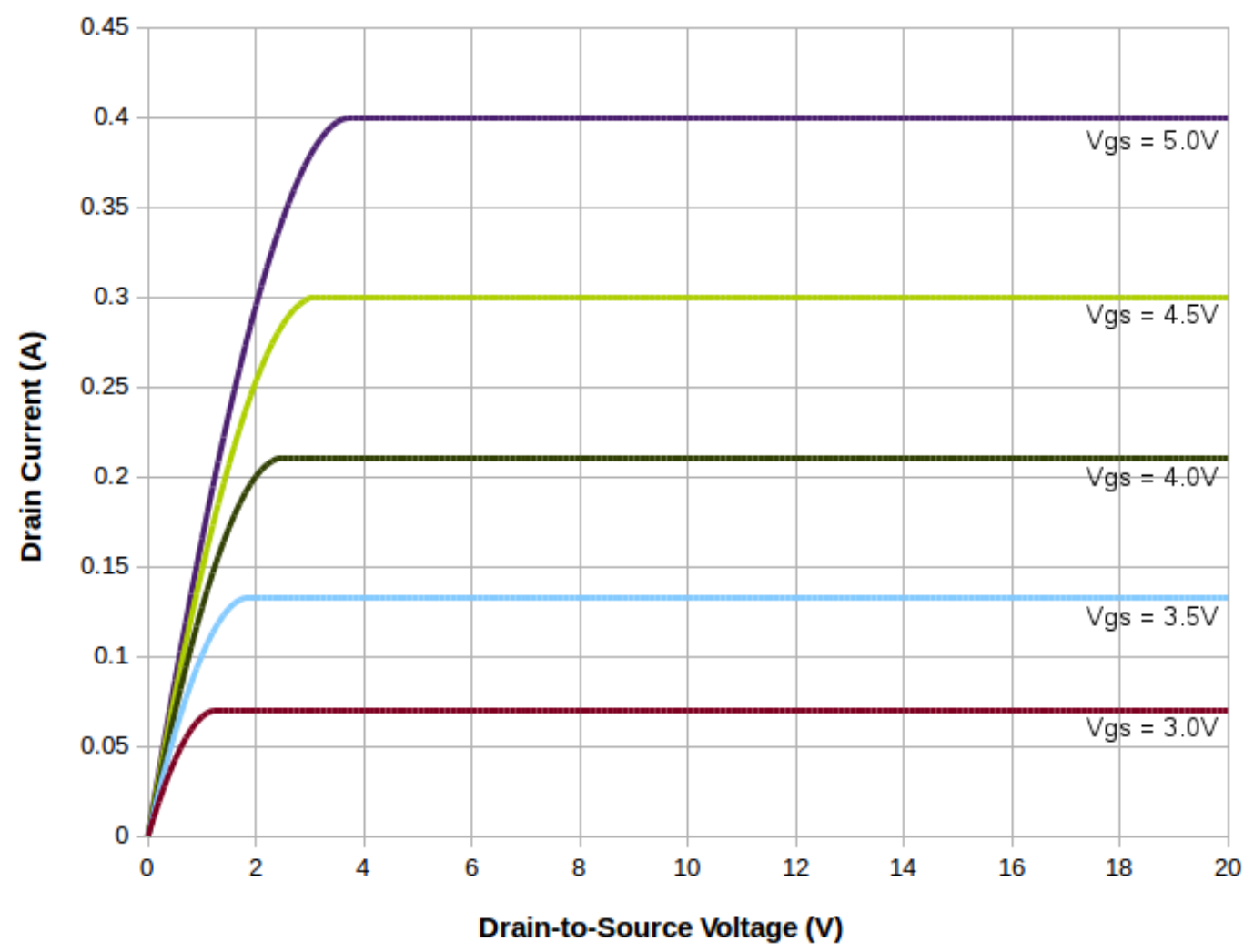

FiguRE 3.2: Simulated I-V Characteristics for a BS170 MOSFET

due to availability, maximum $V_{d s}$ rating of $60 \mathrm{~V}$, and power dissipation capability given its package size. The I-V characteristics for this transistor are shown in Figure 3.2. Using the I-V curves, loadline analysis for a class A amplifier can be performed in order to start the design. Design of this class of amplifier starts by using two simple equations based around the quiescent current and DC supply voltage.

$$
\begin{gathered}
R_{o p t}=\frac{V_{D C}}{I_{\max } / 2}=\frac{V_{D C}}{I_{D C}} \\
P_{o p t}=\frac{1}{2} V_{D C} I_{D C}
\end{gathered}
$$

The two expressions given for Equations 3.2 and 3.3 can be used under the assumption 
that the amplifier efficiency is 50\% [27]. According to the transistor datasheet, the device can handle up to $500 \mathrm{~mA}$ of continous current draw and dissipate up to $800 \mathrm{~mW}$ [28]. Given a $12 \mathrm{~V}$ power supply, Equation 3.3 can be solved for $I_{D C}$ and subsequently $I_{\text {max }}$. This provides the operating conditions for the driver and a way to draw the load line using a realistic device [29]. However, a method of matching the output impedance to the load is still needed in order to extract the RF power. Conversion of $R_{\text {opt }}$ to a complex impedance can be performed given the following relationships.

$$
\begin{gathered}
Z_{o p t}=R_{o p t}+j X_{o p t} \\
X_{o p t}=\sqrt{R_{o p t}^{2}\left(1-\frac{1}{p^{2}}\right)}
\end{gathered}
$$

Looking at Equation 3.5, the term $p$ refers to the efficiency factor of the amplifier. Being class A offers $50 \%$ efficiency so $p$ has a value of 2 . From here, a complex impedance (Z) can be calculated and matched to a complex load via a matching network [27]. Additionally, load-pull contours could also be plotted on a Smith chart and used for matching purposes. Being a driver stage, there is an advantage in the fact that an impedance higher than $50 \Omega$ for driving the next stage can be used mainly since the next stage will also be a MOSFET stage. Increasing the load seen at the output of the driver stage to a few hundred ohms could effectively produce a larger voltage swing, thus providing more voltage gain at the output of the next stage depending on the drive requirements of the MOSFET. Figure 3.3 gives a simplified schematic for the driver stage to be designed using the theoretical principles outlined in this section. The value of R2 at the gate of the BS170 could also be reduced to lower the threshold 


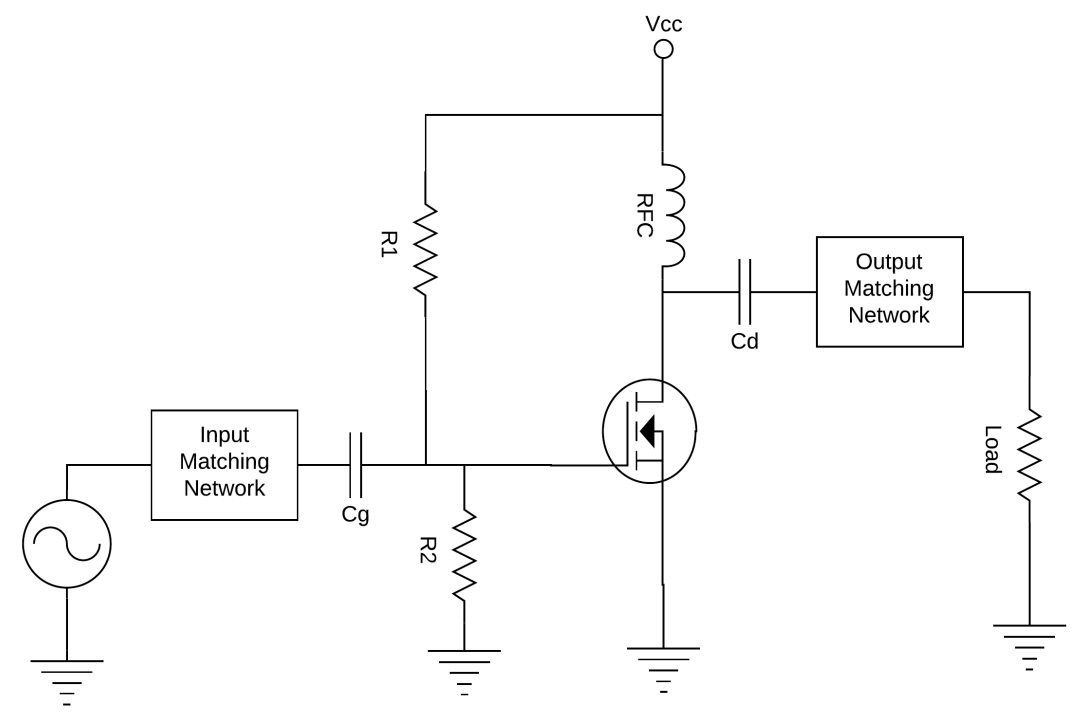

Figure 3.3: Simplified Schematic for a Class A Driver Stage

voltage effectively pushing the amplifier into class- $\mathrm{AB}$ operation if increased efficiency is needed [30].

\subsection{Final Stage}

For the final RF amplifier stage, exploitation of amplifier efficiency would be desired to reduce the power dissipated and still be able to achieve a higher output power level. Another consideration comes from how the transmitting amplifier is switched into the on state in that the input signal from the LO is switched on or off at the input to the driver stage. Choice of class $\mathrm{B}$ or $\mathrm{C}$ would be a likely candidate here since the current draw would be limited or practically zero if no signal is present at the input [32]. With the driver configured for class-A operation, some power will be constantly dissipated regardless of whether or not the system is transmitting. Reducing the conduction angle will only help keep unnecessary power dissipation to a minimum in the receive state. The design of the transceiver is intended only for CW, so linearity is less of a concern over output power [31]. Therefore, an IRF510 power MOSFET was chosen for the final 
transmitter stage. This transistor has a maximum drain-to-source voltage $\left(V_{d s}\right)$ of 100 $\mathrm{V}$ and can sustain a continuous drain current of $4 \mathrm{~A}$ [33].

Assuming a sinusoidal input to the amplifier, a waveform analysis can be performed to illustrate how efficiency increases with the reduced conduction angle. Using the symmetry of the input signal, a half conduction angle $(\theta)$ can be taken over the range where the transistor is conducting. This allows for an expression of the quiescent current $\left(I_{C Q}\right)$ in relation to a maximum current $\left(I_{\max }\right)$ and the half conduction angle. The DC and fundamental current components then can be expressed in the form of the Fourier series of the waveform and reduced to the equations 3.6, 3.7, and 3.8.

$$
\begin{gathered}
I_{C Q}=I_{\max } \cos (\theta) \\
I_{D C}=\frac{I_{\max }}{\pi}[\sin (\theta)-\theta \cos (\theta)] \\
I_{0}=\frac{I_{\max }}{2 \pi}[2 \theta-\sin (2 \theta)]
\end{gathered}
$$

Referring back to Equation 3.1 in the previous section, a formula for the maxium efficiency of $\left(\eta_{\max }\right)$ can then be expressed, as shown in Equation 3.9. A plot of this expression as a function of $\theta$ can be seen in Figure 3.4 [32]. As $\theta$ then falls below $\frac{\pi}{2}$, the PA enters class $\mathrm{C}$ operation. This increase in efficiency does come at the price of reduced gain since less and less of the input signal is being represented at the output. From the figure then, it would seem the best course of action to bias the amplifier in 


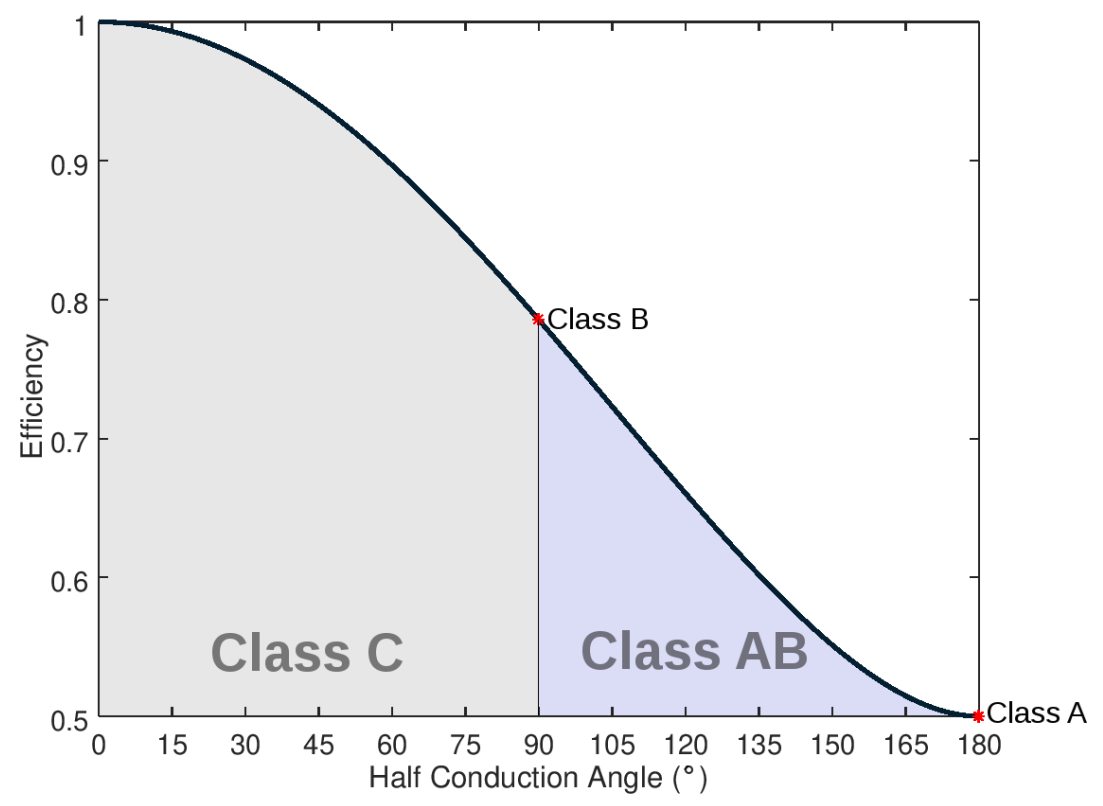

Figure 3.4: Plot of Theoretical Amplifier Efficiency

such a way to keep the conduction angle as close to the class B point without going into class B operation.

$$
\therefore \eta_{\max }=\frac{2 \theta-\sin (2 \theta)}{4[\sin (\theta)-\theta \cos (\theta)]}
$$

By applying no bias to the gate of the IRF510 from the power supply, the threshold voltage should be high enough to keep the amplifier well in the class $\mathrm{C}$ operation region. The output from the driver should be able to reach output levels of $5 \mathrm{~V}$ or higher to effectively turn on the transistor. However, a Zener diode connect to a ground resistor could also be placed at the gate to provide a short time constant that varies as the voltage changes. A voltage develops as a result of the charge stored in the junction capacitance. This effect has the ability to alter the quiescent current over the transition into cutoff reducing amplifier efficiency. A simplified schematic is then shown in Figure 3.5 . 


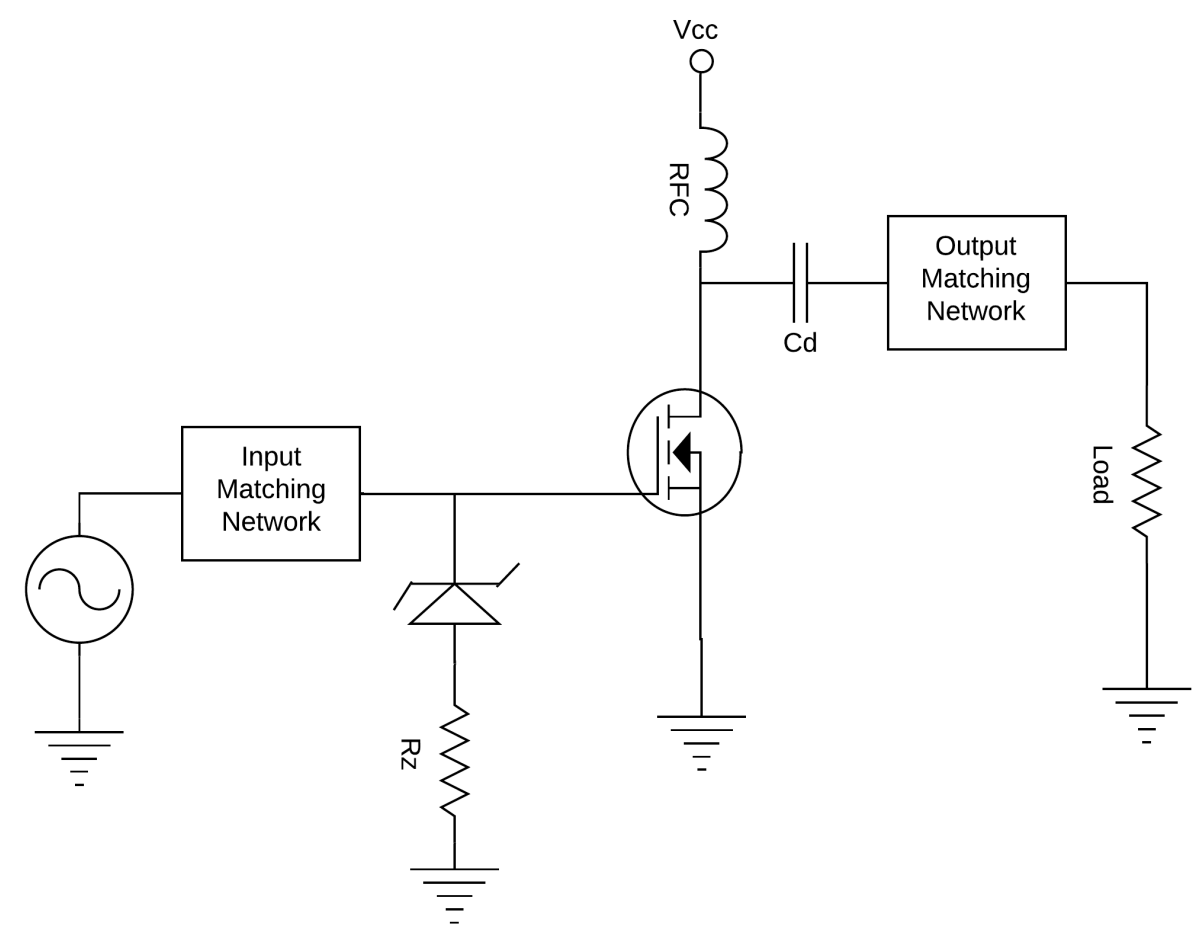

Figure 3.5: Simplified Schematic of the IRF510 Final Stage Power Amplifier

\subsection{Digital Transmit/Receive Switching}

For the last section in this chapter, the method of switching still needs to be addressed for the transceiver system to be functional. In the block diagram introduced in Figure 1.1, a number of single-pole switches can be seen connected to the MCU. These switches need to be active RF switches with high isolation that respond to a digital control signal. A common method of doing this would be a common-gate JFET sharing the same supply bus as the MCU. Due to pricing, availability, and package size, an Atmel ATTiny85 was chosen for the MCU. This chip can use a 5 V supply and comes in an eight-pin dual inline package (DIP). The single-pole JFET switches then can share the supply voltage with MCU and a control signal can effectively switch the transistor on and off. The drain-to-source resistance of a JFET is generally determined by the approximation given in Equation 3.10, where $g_{m}$ represents the forward 


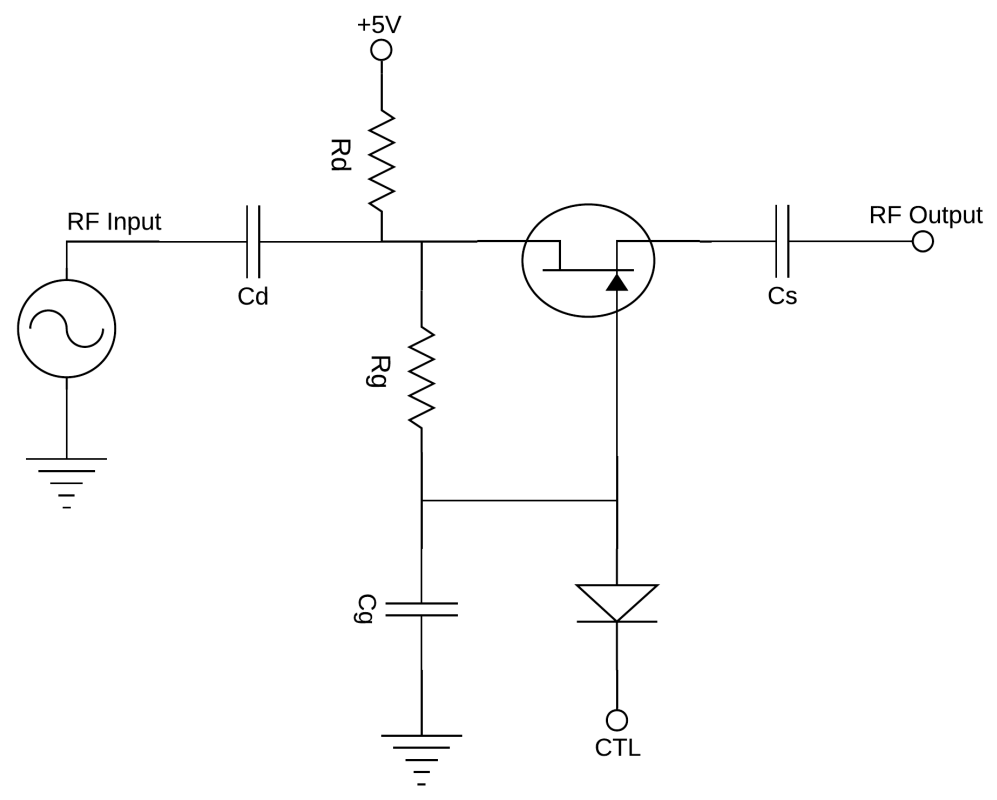

FIGURE 3.6: Schematic for RF Switch Using a Common-gate JFET Amplifier

transconductance of the transistor [17]. A J310 has a typical $g_{m}$ of $14.1 \mathrm{mS}$ even with a minimal current draw, which translates to an impedance of around $70 \Omega$ [34] and an operable frequency range well into the UHF region. This makes it a good candidate for use as an RF switch.

$$
R_{s} \approx \frac{1}{g_{m}}
$$

Figure 3.6 shows the schematic for the RF switch. Two capacitors provide DC blocking at the input and output of the switch. The $+5 \mathrm{~V}$ supply is connected to the drain resistor. The CTL port is where the digital control signal connect to the MCU. As the control signal switches between high and low states, $R_{g}$ and $C_{g}$ create an analog time constant that creates a small delay as the signal switches. Component values can be determined experimentally to minimize pops or clicks that could wind up at the output of the audio amplifier. $R_{g}$ also acts as a pull-up resistor, keeping the gate voltage high 


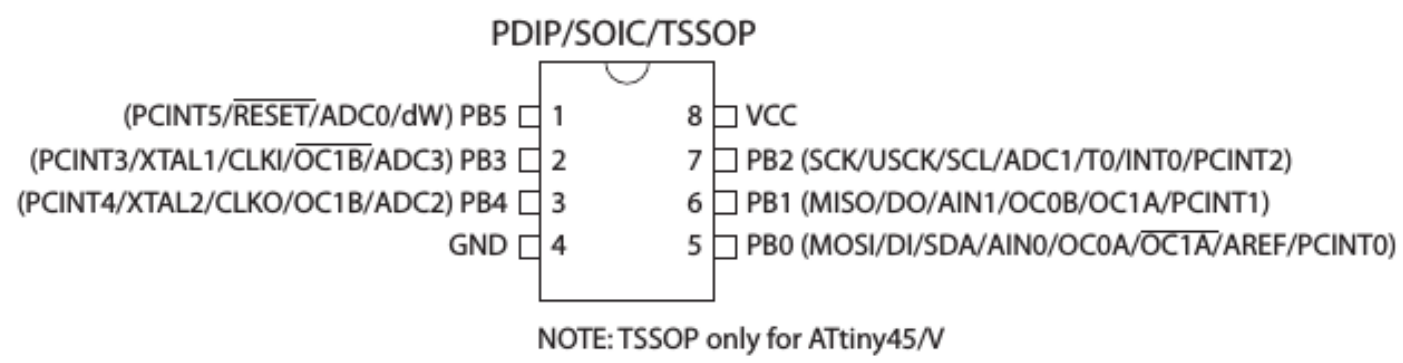

FiguRe 3.7: Pinout for the ATTiny85 8-bit Microcontroller

and keeping the switch ON with a high logic value.

The ATTiny85 AVR microcontroller has an 8-pin package featuring $8 \mathrm{kB}$ of in-system programmable flash memory and six programmable I/O pins available for use as digital control lines [35]. This allows for the creation of a transmit (TX), receive (RX), and frequency adjust (ADJ) bus. The ATTiny85 also includes an 8-bit high speed timer/counter with separate prescalar, which can be utilized for the sidetone oscillator (SOSC). The switch control pin is assigned to a 10-bit ADC, which will receive two separate voltages for the keyer and spot controls. Figure 3.7 shows the pinout and their various functions. 


\section{Chapter 4}

\section{Receiver Module Characterization}

\subsection{Local Oscillator}

In this chapter, the individual receiver modules highlighted in Figure 2.1 are designed using the criteria and methods outlined in Chapter 2 and then simulated using a circuit simulator software known as LTSpice. Once adequate performance was achieved in the simulator, individual modules were assembled and characterized on a bare piece of copper-clad FR4 laminate. The results for each module are given in the following sections.

Being a critical peice of the entire transciever for both transmit and receive, the majority of the time spent on development was done with the LO. After many variations of the simplified design given in Figure 2.6, suitable performance was obtained. The simulated schematic shown in Figure 4.1 produced oscillations in the frequency range but required some manipulation to produce the expected result of a crystal stabilized oscaillator. One such idealization includes the current source (I1). This current source acts as an impulse function and provides a short pulse of current to reduce simulation time needed to create the oscillations. Using this technique produces a signal at the output in less than $200 \mu s$ and required only about $10 \mathrm{~s}$ of processing time during the simulation. 


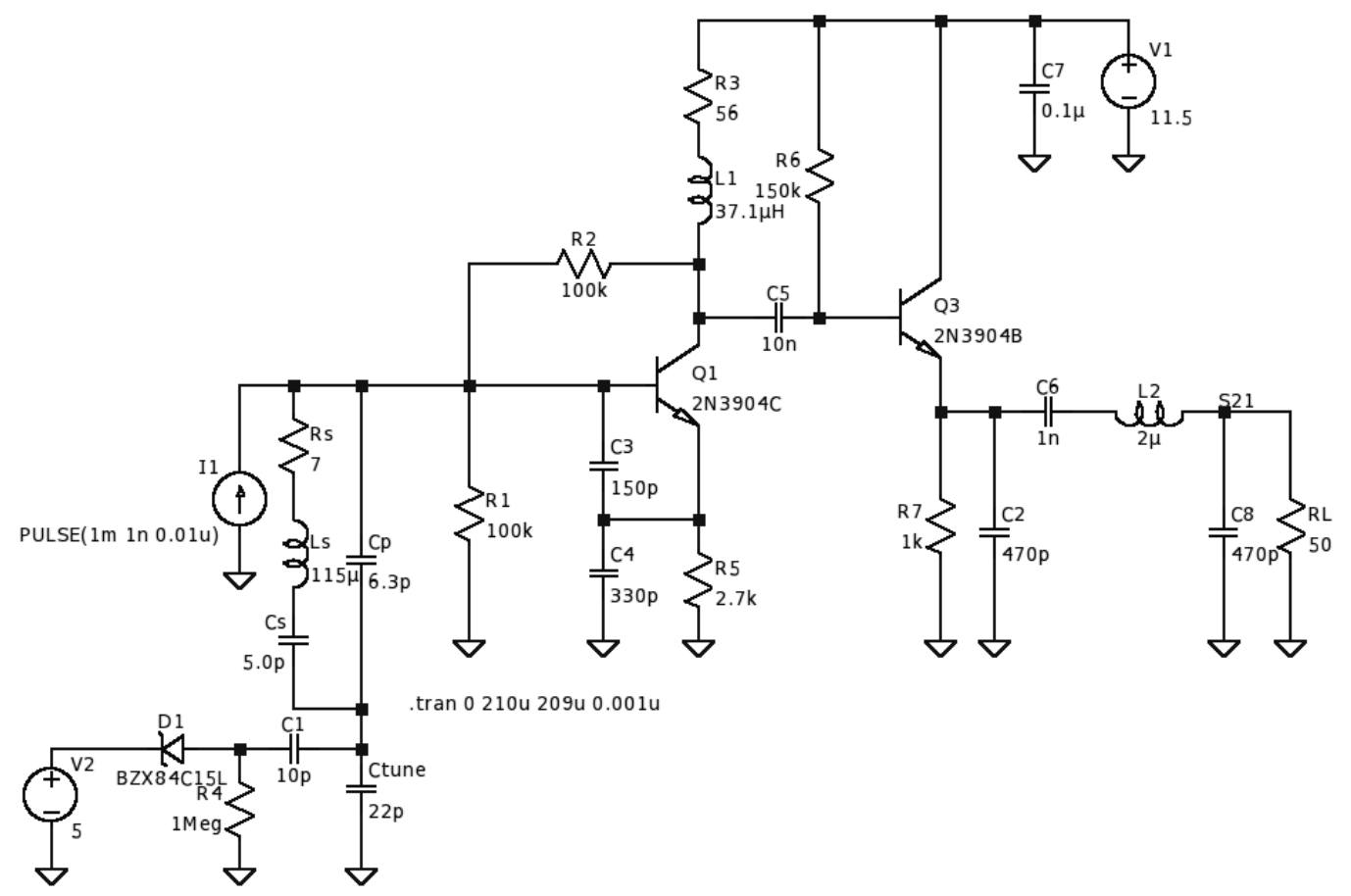

Figure 4.1: LTSpice Schematic for the Local Oscillator

In order to acheive some level of accuracy with the non-linear devices in the circuit, a model for the 2N3904 transistor was downloaded from ON Semiconductor's web site. Three physical transistors were selected and had their DC characteristics compared with simulator. Three copies of the supplied model were then made with each having their beta values adjusted to match the measured values of the physical ones. This at least created similar but not identical transistors for the purposes of simulation. The simulated fast Fourier-transform (FFT) of the oscillator output is given in Figure 4.2.

The parallel crystal resonators shown in the simplified schematic proved to be somewhat difficult to simulate in LTSpice. Therefore, pulling range and automatic frequency offset range were determined experimentally. In fact, the value of $L_{s}$ should be in the $m H$ instead of $\mu H$ as given in Figure 4.1. By reducing the value of $L_{s}$ and increasing the value of $C_{s}$, this is basically the same tactic employed by placing multiple crystal resonators in parallel, but the values needed to produce the oscillation in the simulator are unrealistic and result in overly optimistic performance. In simulation, the focus 


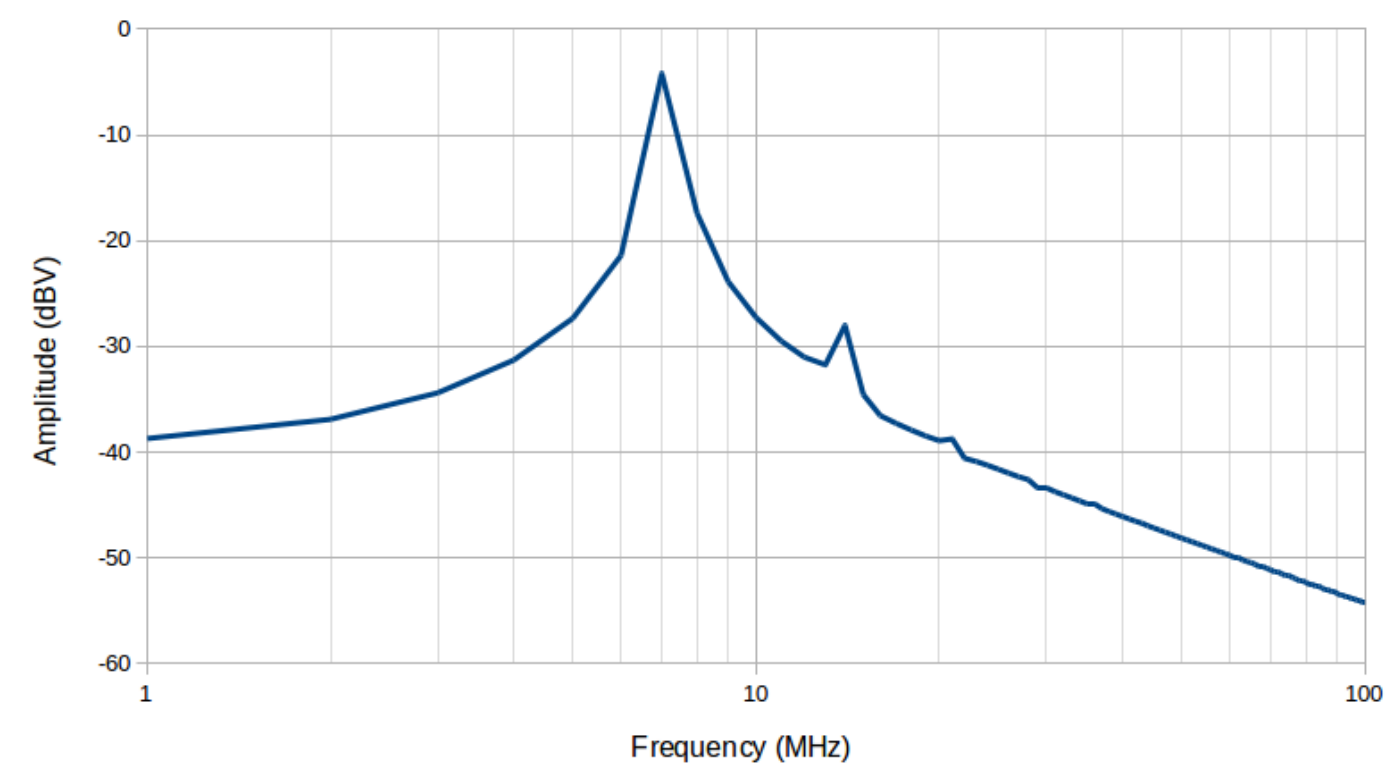

Figure 4.2: Simulated FFT of Local Oscillator Output

\begin{tabular}{|l|l|l|}
\hline Parameter & Simulated & Measured \\
\hline \hline$I_{D C}$ & $8.2 \mathrm{~mA}$ & $10.6 \mathrm{~mA}$ \\
\hline$P_{D C}$ & $95 \mathrm{~mW}$ & $125 \mathrm{~mW}$ \\
\hline$V_{\text {Peak }}$ & $935 \mathrm{mV}$ & $720 \mathrm{mV}$ \\
\hline$P_{R F}$ & $9.71 \mathrm{dBm}$ & $7.15 \mathrm{dBm}$ \\
\hline
\end{tabular}

TABLE 4.1: Simulated/Measured Comparison Table for Local Oscillator

was then getting the most power output and determining the functionality of the frequency offset. Table 4.1 gives a comparison of measured performance metrics versus simulated.

Figure 4.3 shows the build of the LO while Figure 4.4 shows the power output of the fundamental frequency using a $7.055 \mathrm{MHz}$ crystal. One interesting thing regarding the measured performance of the LO was how the power output changed as the frequency was pulled. If the series inductor value was too large, the power output would drop considerably towards the end of the pulling range. Using $+4 \mathrm{dBm}$ as the minimum power output needed to drive the ADE-1 mixer, an FT37-67 toroid was wound with 30 turns and then backed off experimentally until and adequate drive level was achieved across the tuning range. 


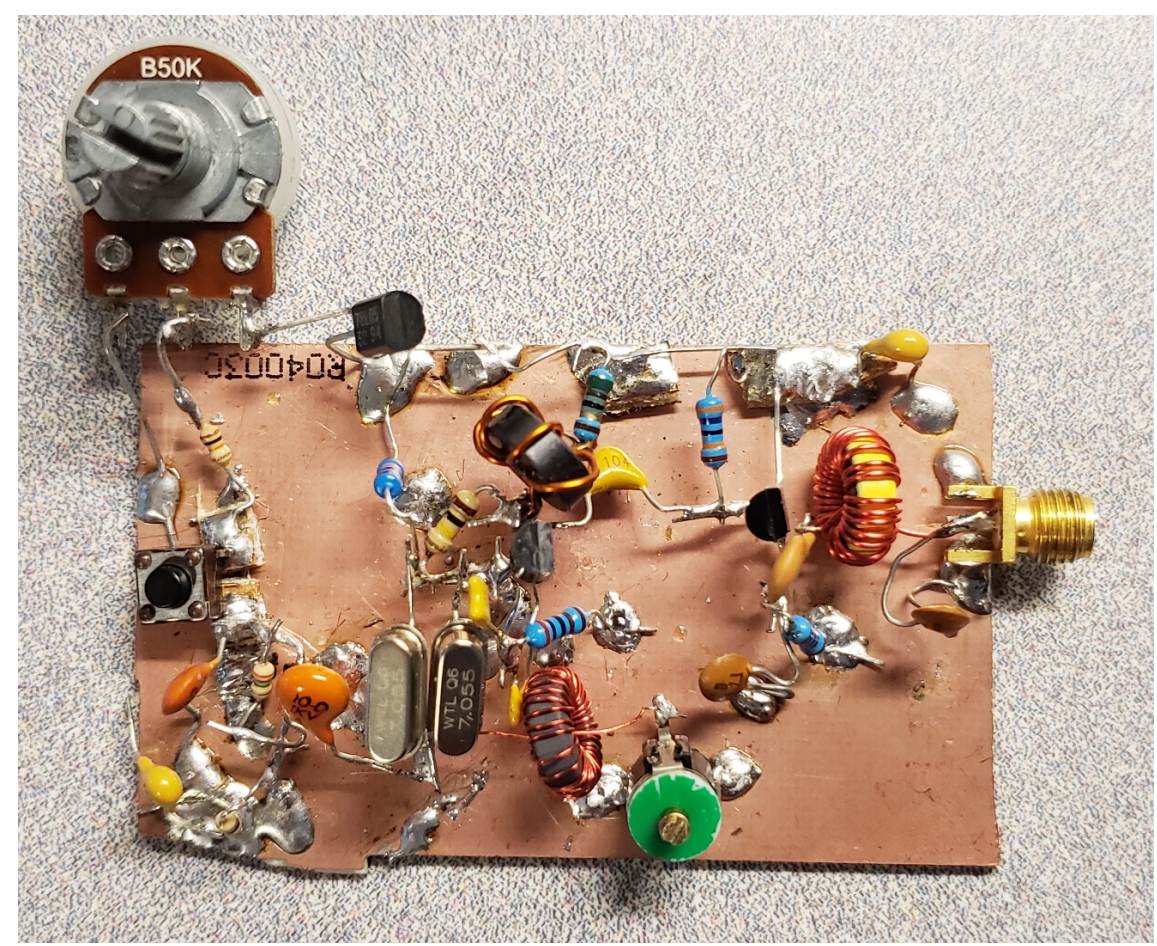

Figure 4.3: Build of Local Oscillator Module

Mkr1 7.053 $2 \mathrm{MHz}$

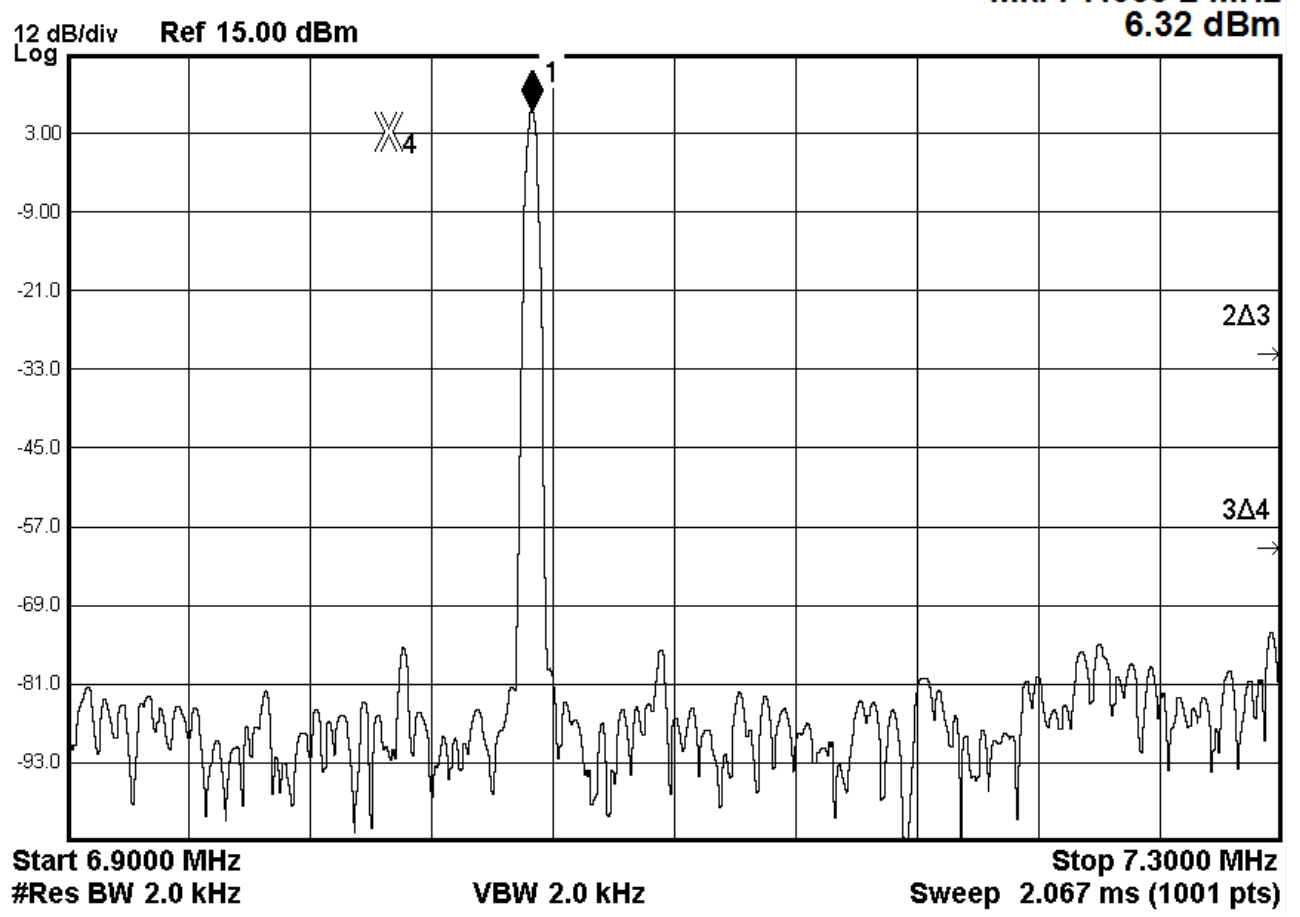

Figure 4.4: Measured Power Output of Local Oscillator Build 


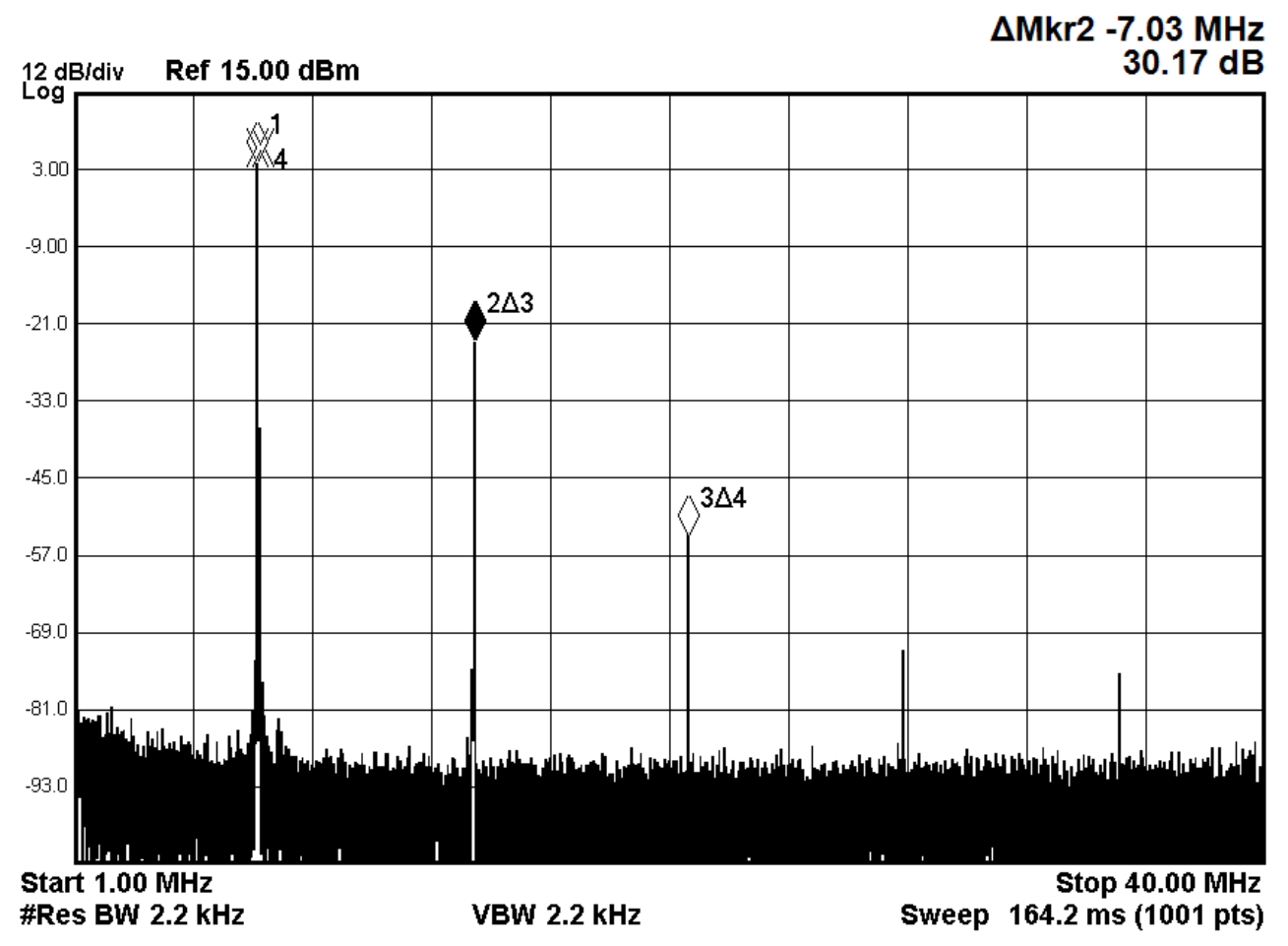

Figure 4.5: Measured Harmonic Content of Local Oscillator with Output Filter on Agilent N9030A

A third-order Butterworth filter was placed at the output of the LO to attenuate the harmonic content of the ouput signal. This provided a clean input signal to the ADE-1 at the fundamental frequency. Figure 4.5 shows the harmonic content of the LO up to $40 \mathrm{MHz}$. From the this measurement, the output filter provides $30 \mathrm{~dB}$ of attenuation at the second harmonic and $54 \mathrm{~dB}$ at the third harmonic, which makes for adequate filtering into the product mixer. Harmonics above the third harmonic are attenuated into power levels at $-70 \mathrm{dBm}$ or less, which is far below any useable power level by the mixer. This made for a fairly clean signal for driving the ADE-1 over the region of operation.

\subsection{Low-Noise Amplifier (LNA)}

For the low-noise amplifier, a few different transistor models were used to develop the design in simulation. Ultimately, the designs using the 2N3904 and BC547C devices 


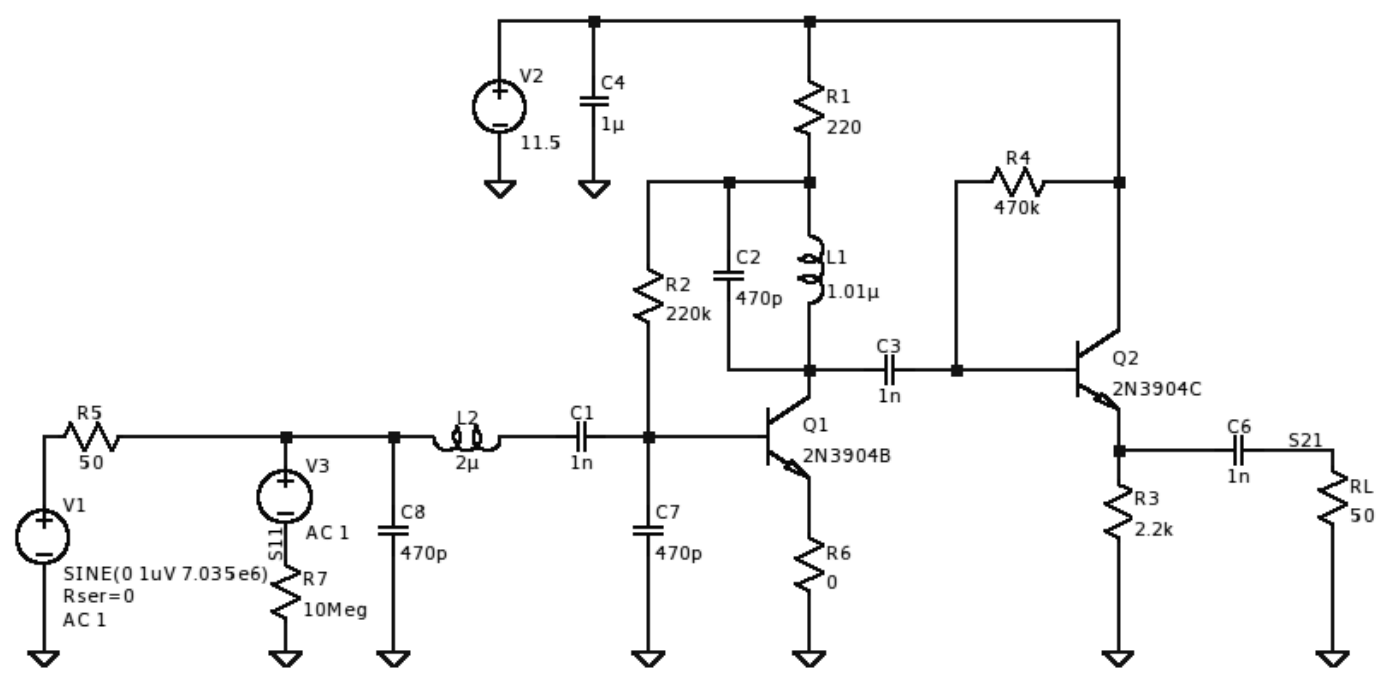

Figure 4.6: LTSpice Schematic for the Single-tuned Low-noise Amplifier

were built and characterized since both devices were readily available. The 2N3904 design provided the most gain over the range of operation by more than $10 \mathrm{~dB}$ and was included in the final design for this reason. The schematic capture shown in Figure 4.6 shows the final design used for the transceiver system. The simulated LNA circuit produced a quiescent current draw of around $7.3 \mathrm{~mA}$ for both stages with $11.5 \mathrm{~V}$ applied to $V_{C C}$. By comparison, the measured current on the module came in sightly higher than the simulated current at $9.4 \mathrm{~mA}$. A picture of the LNA module is shown in Figuer 4.7.

Figure 4.8 provides a plot of the simulated S-parameters provided from LTSpice. The plot of $S_{21}$ shows gains of more than $40 \mathrm{~dB}$ over the range of operation. Attenuation of the input signal at $1 \mathrm{MHz}$ was less than ideal having a simulated gain of $12.9 \mathrm{~dB}$. However, the LNA starts to attenuate signals above $14.4 \mathrm{MHz}$, which contributes to any filtering done after the antenna. The plot of $S_{11}$ shows a good $50 \Omega$ match around $7.15 \mathrm{MHz}$ and the best match at $7.82 \mathrm{MHz}$, but this was less of a concern being that the LNA doesn't provide much power to the load. Gain was the key performance metric 


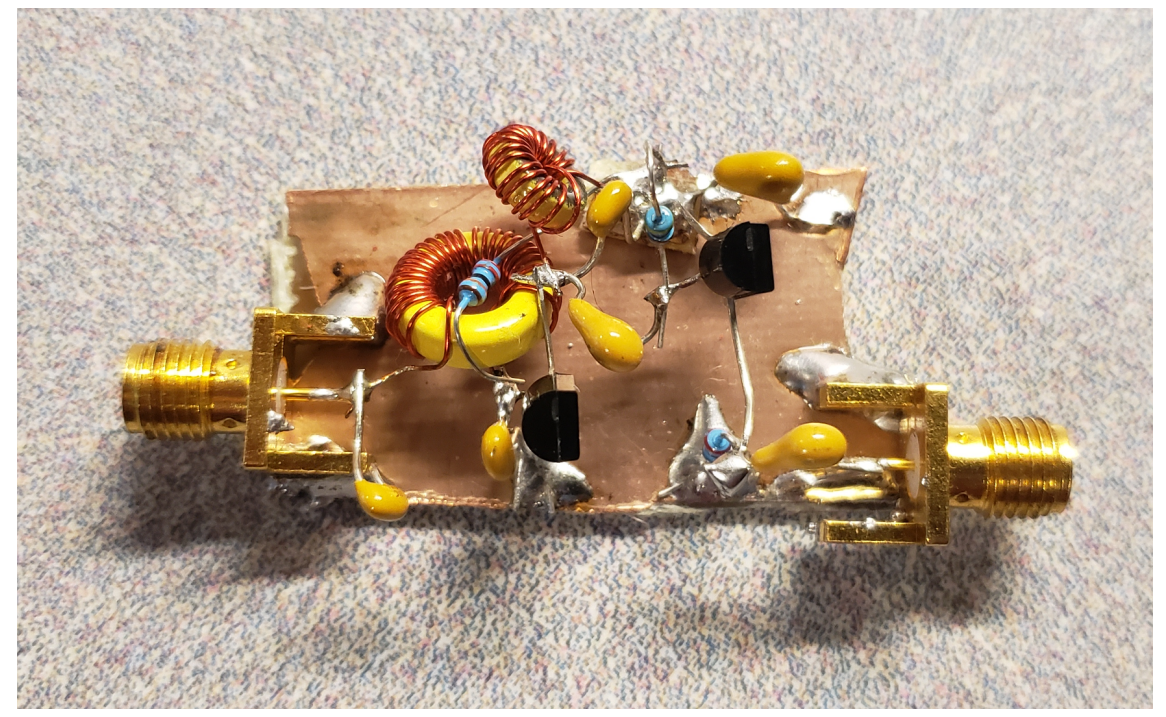

Figure 4.7: Build of Low-noise Amplifier Module

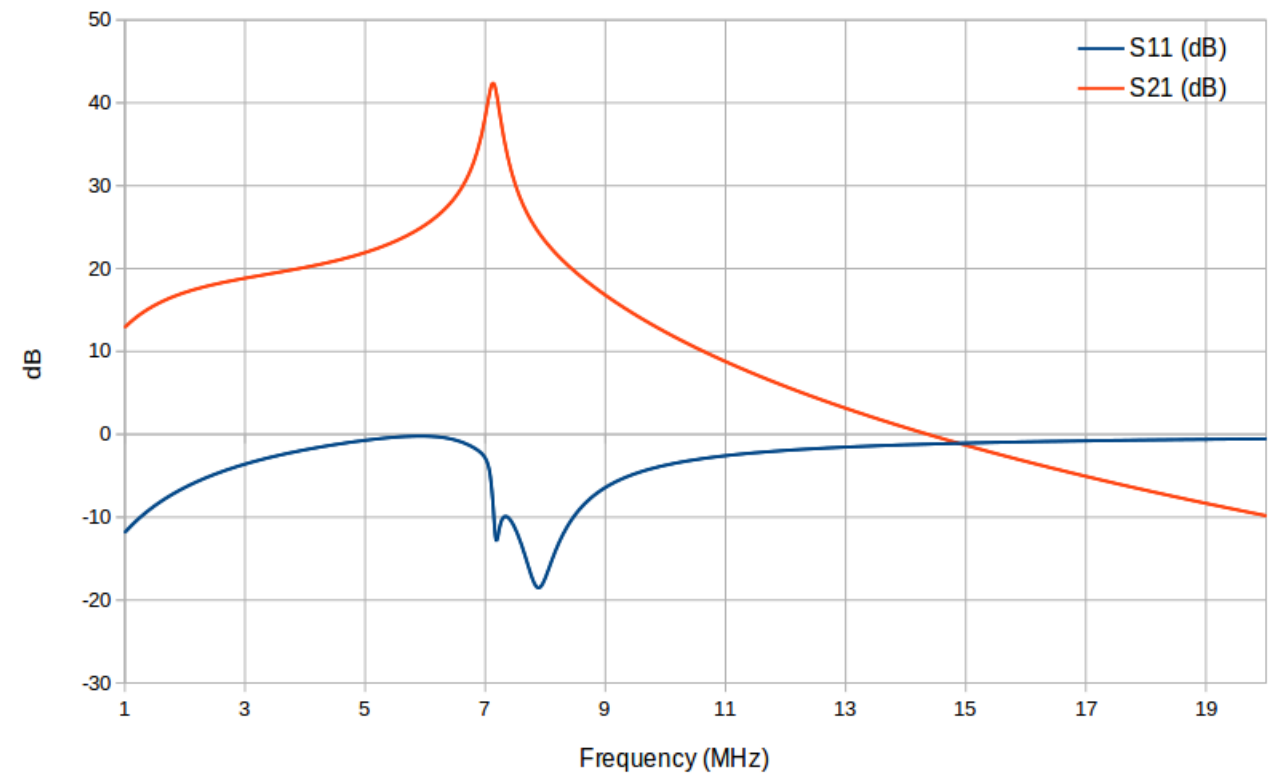

FigURE 4.8: Simulated S-parameters for Single-tuned LNA

utilized for the purpose of the transceiver design.

Comparing measured performance with the simulation, Figure 4.9 shows the measured S-parameters of the LNA module as measured on a vector network analyzer (VNA). The inductor labeled L1 in Figure 4.6 was wound manually on a T37-25 toroid and likely produced a value slightly higher than $1.01 \mathrm{uH}$ given the $S_{21}$ plot shown. However, the overall performance was still adequate for the purposes of the design, yielding 


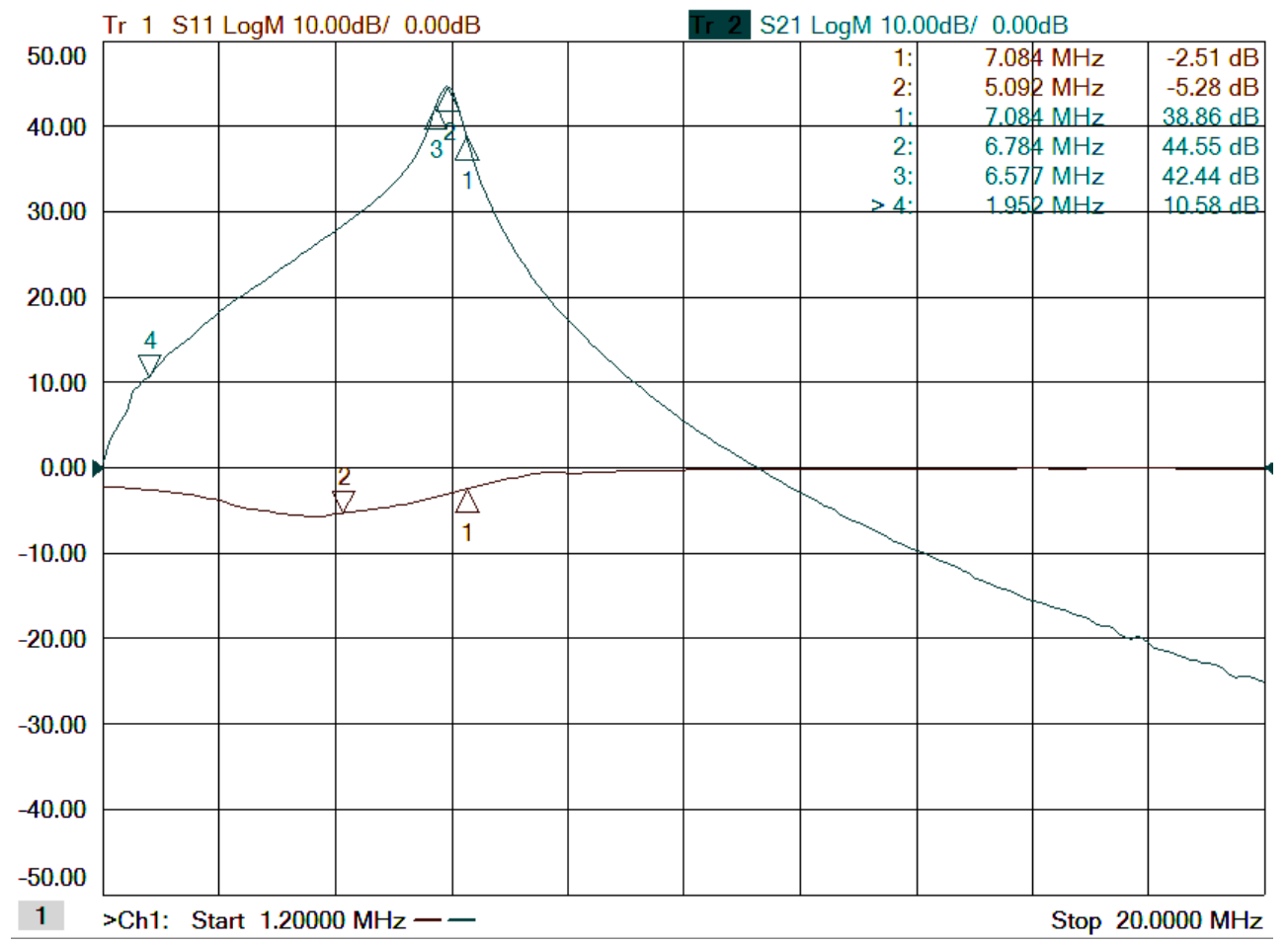

Figure 4.9: Measured S-parameters for Single-tuned LNA Using Agilent E5071C VNA

more than $38 \mathrm{~dB}$ of gain over the band of operation. Additionally, the measured data shows more attenuation at $1 \mathrm{MHz}$, which is benficial at keeping strong signals in the AM broadcast band from overloading the receiver. The plot of $S_{11}$ didn't match the simulation results hardly at all, but performance is still likely adequate for the intended use of the LNA.

\subsection{Audio Amplifier}

As mentioned in Chapter 2, the design of the audio output stage for the receiver revolves around an NE5532 dual op-amp. A schematic of the design simulated in LTSpice is shown in Figure 4.10. The first op-amp (labeled U2) acts as an active first-order low-pass filter having a voltage gain of around $50 \mathrm{~dB}$. The input to the second stage is driven by the other half of the NE5532 (labeled U1), which supplies voltage gain for the complementary-symmetry output stage created by a pair BC547 and BC557 


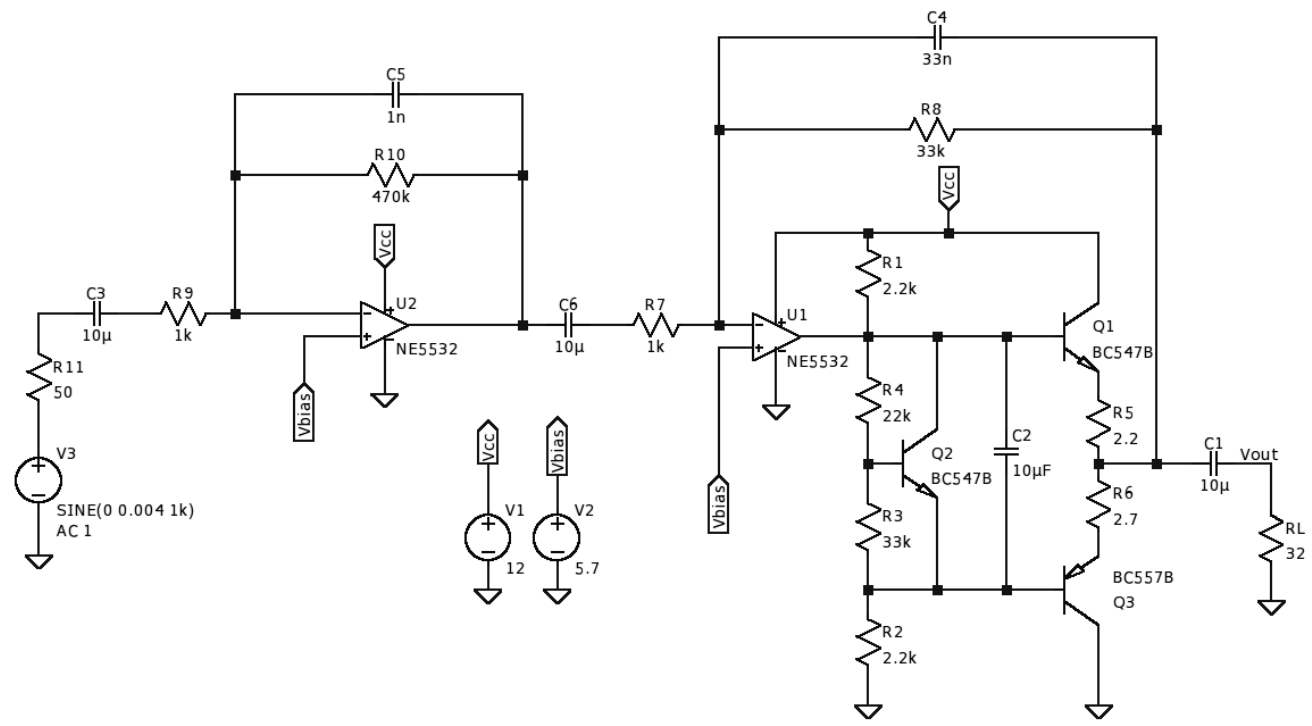

Figure 4.10: LTSpice Schematic for the Receiver Audio Stage

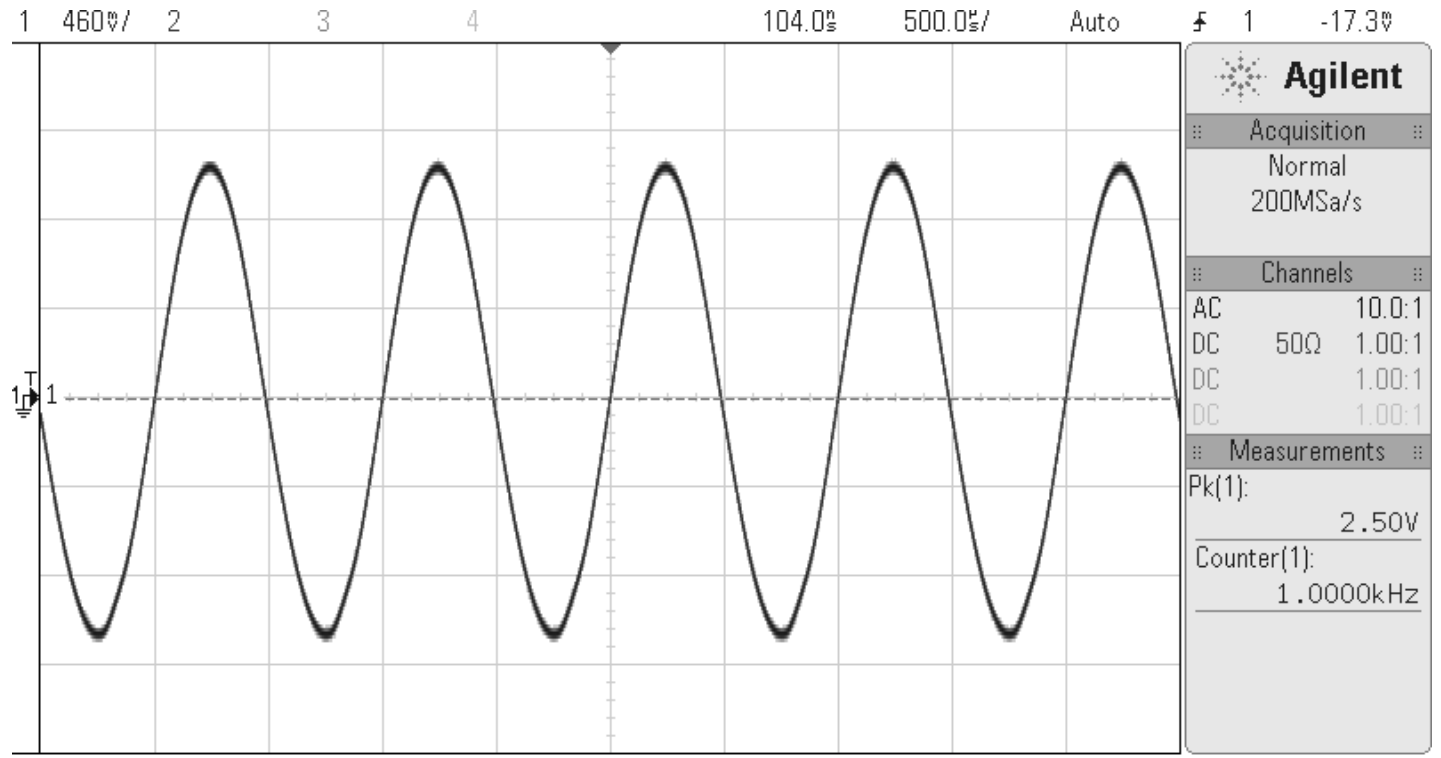

Figure 4.11: Audio Output Capture at $1 \mathrm{kHz}$ into a $32 \Omega$ Load Using Agilent DSO-X $3040 \mathrm{~A}$

transistors (Q1 and Q3 respectively). Q2 sets the bias point both Q1 and Q3 and results in a class- $\mathrm{AB}$ output stage for delivering audio to the speaker or headphones. With a $32 \Omega$ load, the amplifier provided more than $90 \mathrm{~mW}$ at $1 \mathrm{kHz}$. That's enough to exceed the threshold of pain in most earphone applications and enough to drive a small speaker [36]. A time domain capture of the measured output from the circuit built on the module can been seen in Figure 4.11. 


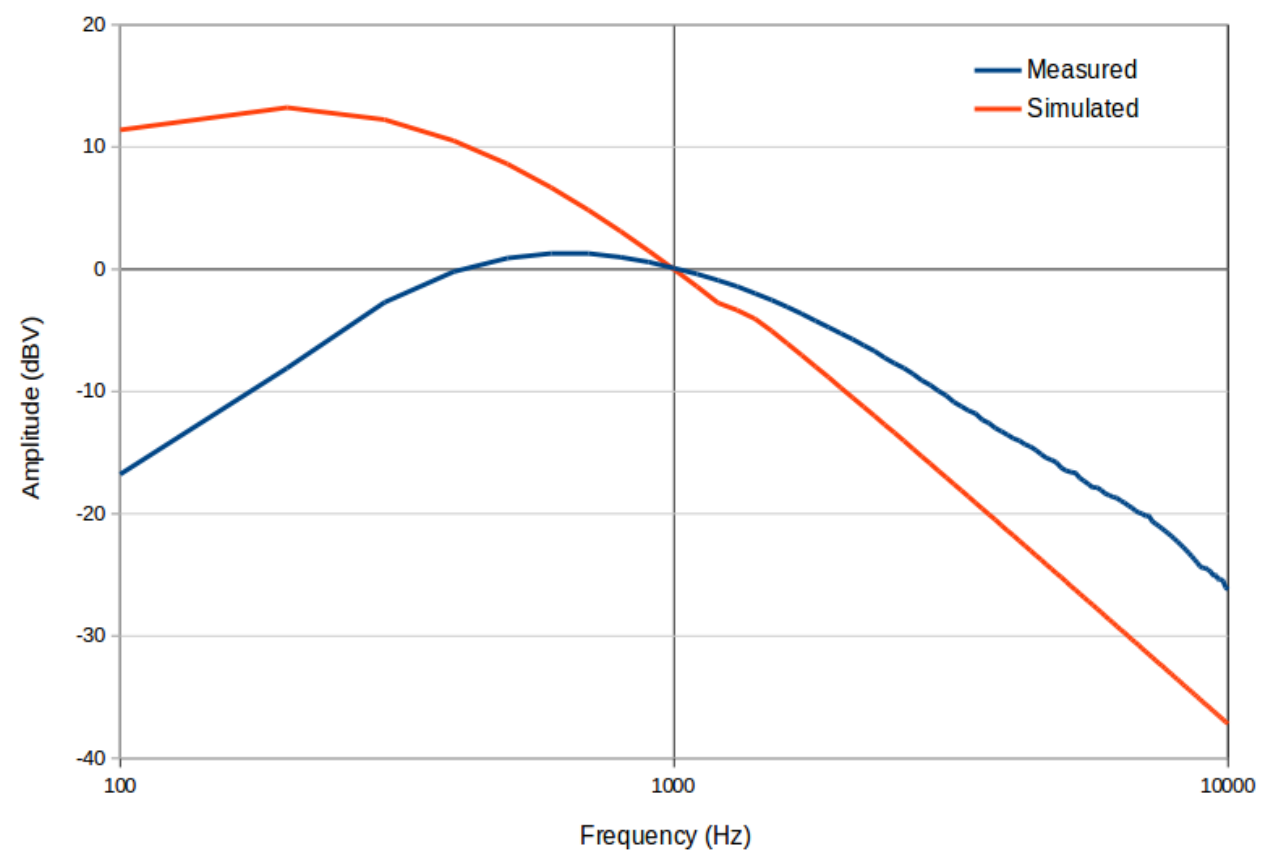

Figure 4.12: Simulated and Measured Amplifier Frequency Response Normalized at 1 $\mathrm{kHz}$

The plot shown in Figure 4.12 gives a comparison of the measured and simulated frequency response of the amplifier. The output was normalized to $1 \mathrm{dBV}$ at $1 \mathrm{kHz}$ at the output of the amplifier and then the frequency was swept from $100 \mathrm{~Hz}$ to $10 \mathrm{kHz}$. Given the filtering on both stages, the frequency response shows a steeper roll off at high frequencies and a lower cuttoff point in simulation when compared to the measured response. However, the response of the amplifier provides $25 \mathrm{~dB}$ of attenuation at 10 $\mathrm{kHz}$.

The low frequency attenuation shown in Figure 4.12 is largely a result of a DC blocking capacitors having a lower value in circuit module. Due to the high gain of the amplifier at low frequencies, large clicks or pops created when keying the amplifier resulted in low frequency oscillations. These oscillations could be halted pretty easily by turning down a potentiometer between $\mathrm{C} 6$ and $\mathrm{R} 7$, but the value of $\mathrm{C} 3$ and $\mathrm{C} 6$ were reduced from $10 \mu F$ to $1 \mu F$ to reduce gain. The values of the $\mathrm{RC}$ network in the feedback path for each stage could also be adjusted to lower the gain while retaining the cutoff 
frequency in the event that oscillations become a problem in the finished transciever. 


\section{Chapter 5}

\section{Transmitter and Switching Module Characterization}

\subsection{Power Amplifier Characterization}

This section outlines the characterization of the transmitter circuitry to be used in the transceiver. As discussed in Chapter 3, the target specification for the power amplifier section swas to be able to deliver a continuous wave $(\mathrm{CW})$ signal with a power level of $30 \mathrm{dBm}$ or more power into a $50 \Omega$ load. The amplifier was designed in such a way to keep quiescent currents to a minimum so that a single JFET switch could be placed between the input to the PA and the LO. This would allow the LO to drive the PA during transmit. The JFET switch had its gate connected to a control bus so that a microprocessor could then turn the amplifier on and off just by muting the input signal.

The PA is composed of two sections: the driver and the final amplifier. For the sake of testing and loading, the PA was assembled originally on a single piece of copper-clad laminate and then tested again on the final printed circuit board. The test results presented here are measured on the final circuit board mounted inside the shielded enclosure, as shown in Figure 6.6. PA measurements varied between shielded and unshielded tests. Figure 5.1 shows the schematic of the circuit simulated in LTSpice. A comparison of the simulated and measured output in the time-domain from a Tektronix 


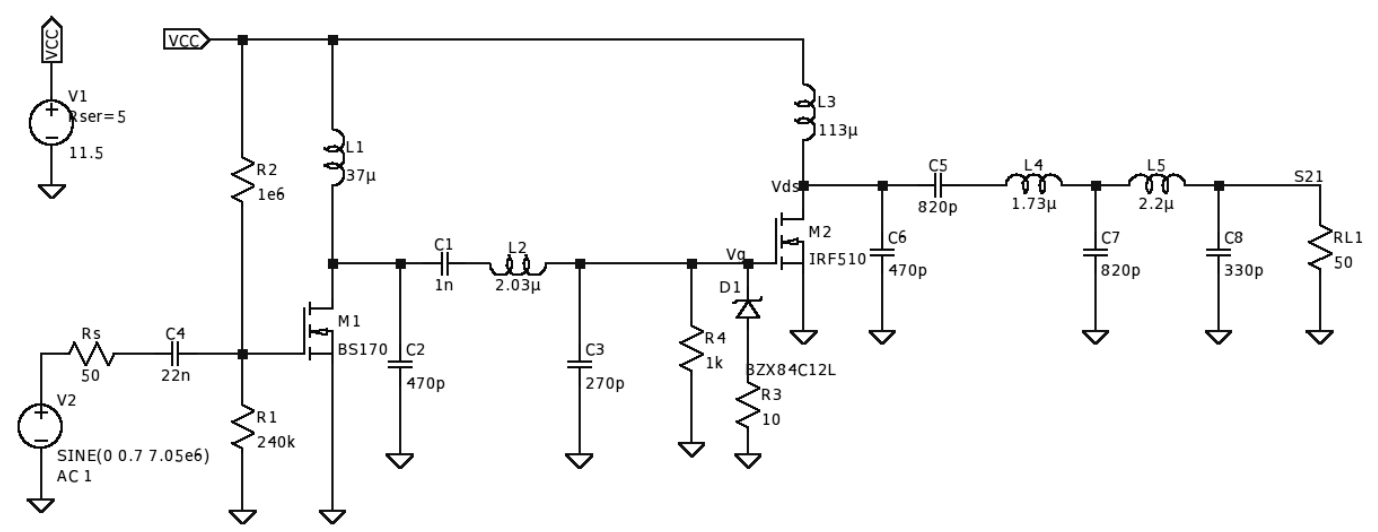

Figure 5.1: LTSpice Schematic of the Power Amplifier Design

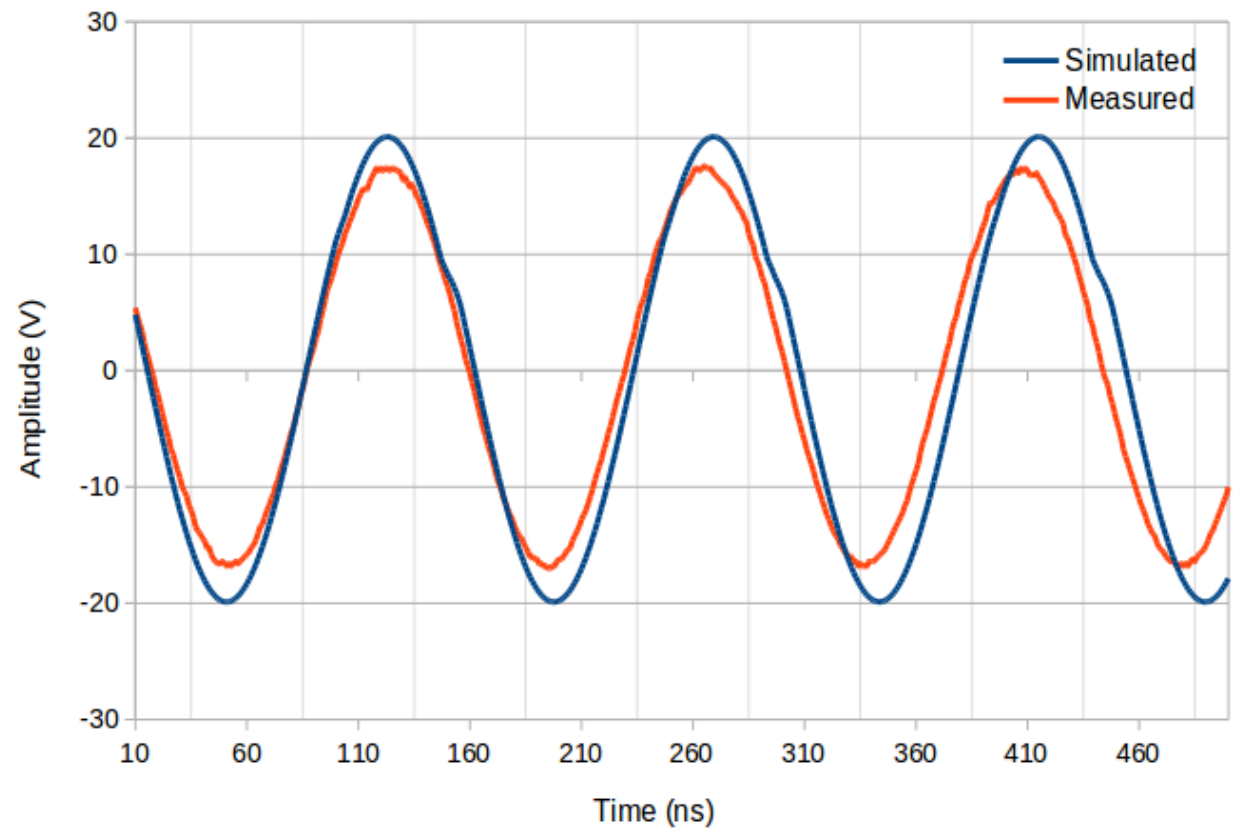

Figure 5.2: Simulated and Measured Comparison of Power Amplifier Output

TDS 360 digital oscilloscope is shown in Figure 5.2. From the plot of the output, the overall power output tracks fairly well, resulting in a difference of $1.3 \mathrm{~dB}$. With a supply voltage a $11.5 \mathrm{~V}$, The output power of the PA was measured at $34.7 \mathrm{dBm}$, which is in the vicinity of 3 watts. Given a DC current of $550 \mathrm{~mA}$, this gives a power-added effeciency (PAE) of $46.5 \%$. This varies from simulation by a large margin since the simulator is able to get more power while consuming only $504 \mathrm{~mA}$ of current. This results in a simulated PAE of $68.9 \%$. 


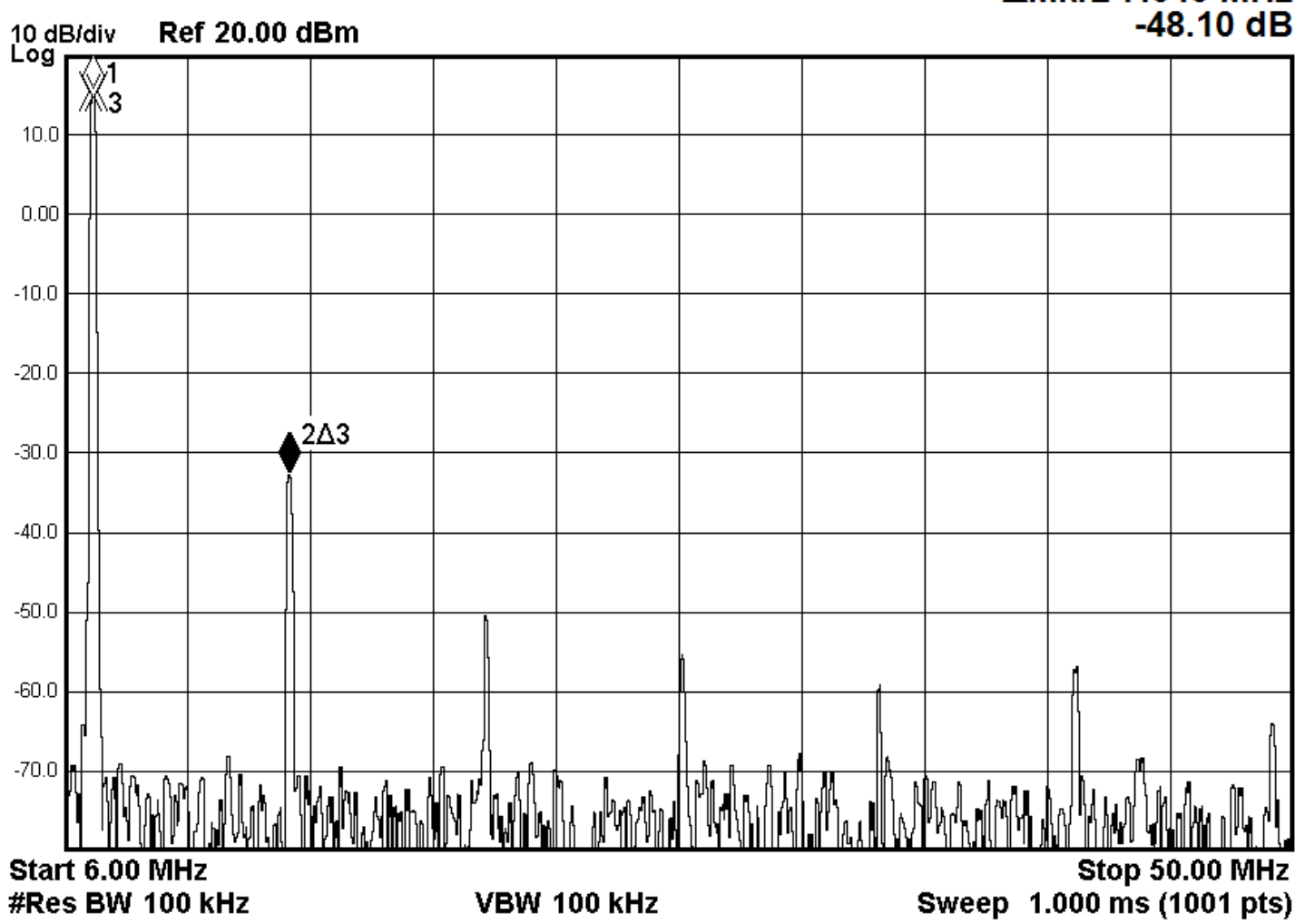

Figure 5.3: Spectral Content of the Power Amplifier Output Measured with Agilent N9030A Spectrum Analyzer

Figure 5.3 displays the measured spectral output of the power amplifier. In this plot, the overall effectiveness of the output filter in attenuating higher order harmonics can be seen. The setup for this measurement included a $20 \mathrm{~dB}$ attenuator on the input of the spectrum analyzer. Therefore, measurements in the plot are $20 \mathrm{~dB}$ below their actual value. The second harmonic of the CW output signal is attenuated by $48.1 \mathrm{~dB}$, resulting in an output power of $-13.4 \mathrm{dBm}$. This is in compliance with the FCC's Part 97 emission standards for transmissions below $30 \mathrm{MHz}$ [37]. Higher harmonics don’t see as drastic of a reduction like that between the fundamental and second harmonic, but power levels fall to or below $-30 \mathrm{dBm}$ and are acceptable. Power levels appear to drop off into the noise floor beyond the seventh harmonic. 


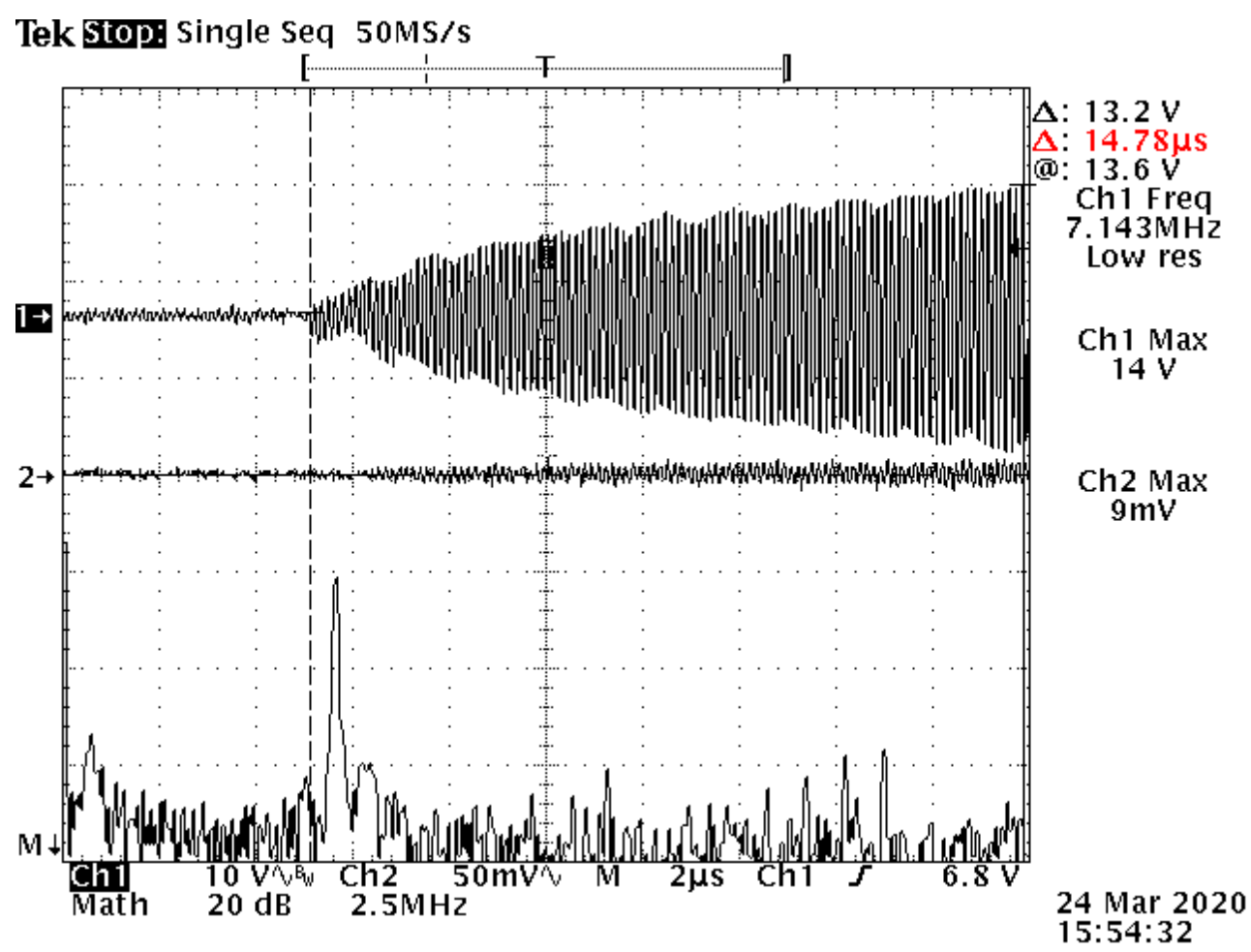

Figure 5.4: Measured Switching Time of JFET Transistor Switch on Tektronix TDS 360 Oscilloscope

\subsection{RF Switch Measurements}

The RF switch design using a J310 JFET transistor shown in Figure 3.6 was also assembled on the printed circuit board and tested by measuring the PA turn-on time. In order to examine the switching time of the transistor alone, the capacitor, $C_{g}$, at the gate of the transistor was removed. This eliminated the short delay introduced by the time constant created by $R_{g}$ and $C_{g}$. The LTSpice simulation results measured a turn on time of $7.5 \mu \mathrm{s}$ and a current draw of $4 \mu \mathrm{A}$. This varied somewhat from the measurements taken on a Tektronix TDS 360 digital-storage oscilloscope as shown in Figure 5.4. The measured turn-on time was more than double the simulation results at $14.8 \mu \mathrm{s}$ and drawing $10 \mu \mathrm{A}$ of current. Despite both measurements being off by a factor of more than double, the differences are still negligible in terms of this design. 


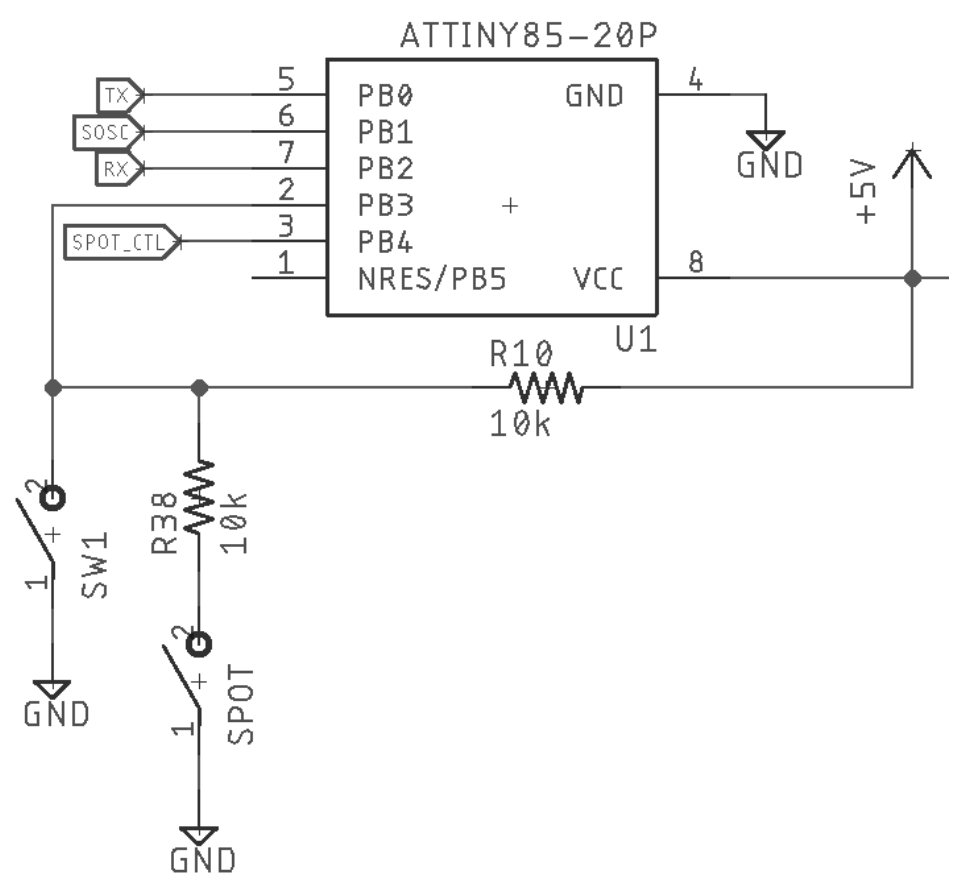

Figure 5.5: Schematic of MCU Transceiver Application Circuit

\subsection{Digital Control}

The final section in Chapter 3 gives the specification of a control bus for each functioning block of circuitry. The schematic in Figure 5.5 shows the electrical configuration and pin assignment for the ATTiny85P MCU. Since the JFET switches have been designed to work with a $+5 \mathrm{~V}$ control signal, there are three pins dedicated as outputs to enable and disable various RF switches. These are 5-volt digital logic output sent from the MCU for manipulating the TX, RX, and SPOT_CTL busses. Pin 2 (PB3) is an input pin on the MCU connected to the 10-bit analog-to-digital converter (ADC). Using the ADC on PB3 is useful since a number of control input signals could then be created by using different voltage levels. In the schematic, R10 acts as a pull up resistor until either the SW1 or SPOT switch is connected. Placing R38 in series with the SPOT switch allows the MCU to see two different voltages, depending on which switch is pressed. This technique was used to create a spot feature allowing the operator to zero-beat the incoming signal by listening on the transmit frequency. 
The application of the spot feature was found to be very useful when operating on the air. When the SPOT switch is connected to ground, a logic high signal is applied to the SPOT_CTL bus. This bus was connected first to a potentiometer and then to the frequency control line on the local oscillator. In operation, a signal can be heard on either the upper or lower side band of the LO frequency. The operator adjusts the tuning knob until the calling station is on the upper side of the LO frequency. Once the station's tone is around 500 to $700 \mathrm{~Hz}$, pressing the SPOT button on the front of the radio switches the LO from the receive frequency to the transmit frequency. The transmit frequency will always be above the receive frequency. The potentiometer connected to the SPOT_CTL bus then acts and a transmit incremental tuning (XIT) control in order to best zero-beat the incoming station. Once the calling station can no longer be heard, releasing the SPOT switch returns the LO to the receive frequency where the station can again be heard.

\subsection{MCU as a Side-tone Oscillator}

In order to have audible feedback when keying the transceiver, pin 6 (PB1) was connected to the MCUs internal Timer1 and configured for PWM mode to act as a sidetone oscilltor. A 32-byte look-up table was created in the program to contain amplitude values of a sine wave. The PWM mode of the ATTiny 85 produces an output compare match when the value of the output compare register is reached. By setting the output compare register to the amplitude value of the waveform in the look-up table, the PWM output of the pin has a varying duty cycle at the clock frequency. A 14-bit prescalar can by applied to the clock frequency, effectively slowing down the rate of the PWM signal. With a 32-byte waveform, the PWM frequency could be at least 32 times the audio frequency. Adding a very lossy first order audio filter on the output, 
the high frequency PWM signal can be removed, leaving an 8-bit audio signal. While this signal isn't by any means a pure sine wave, enough filtering makes it sound good enough for feedback purposes. A clear tone of $600 \mathrm{~Hz}$ is heard on the output of the audio amplifier whenever the transmitter is keyed up. 


\section{Chapter 6}

\section{Results and Discussion}

\subsection{Schematic and Board Layout}

This chapter primarily deals with the construction and board-level testing of the transceiver system as a whole. A small 3.5 by 4 inch aluminum enclosure houses the double-sided printed circuit board (PCB), which contains all the transceiver circuitry. The footprint of the board has the dimensions of 3.5 by 3 inches in order to make space for the controls and connections needed on the front and back panels. The schematic capture and PCB layout was drafted in EagleCAD and are shown in Figures 6.1 and 6.2 respectively. PCB manufacturing was done by Osh Park, and assembly began on arrival. The first fully assembled transceiver unit was actually stolen after being field tested and left overnight in a vehicle. A second assembly was built, which provided most of the data presented in this document.

\subsection{Board-level Testing}

Initial bring-up of the board started by assembling the LO circuitry so that voltage measurements of its output could be taken and troubleshooted before adding additional components. The original board layout had only two crystal resonators and was adapted to include four in a later revision. Due to time constraints, the routing around 


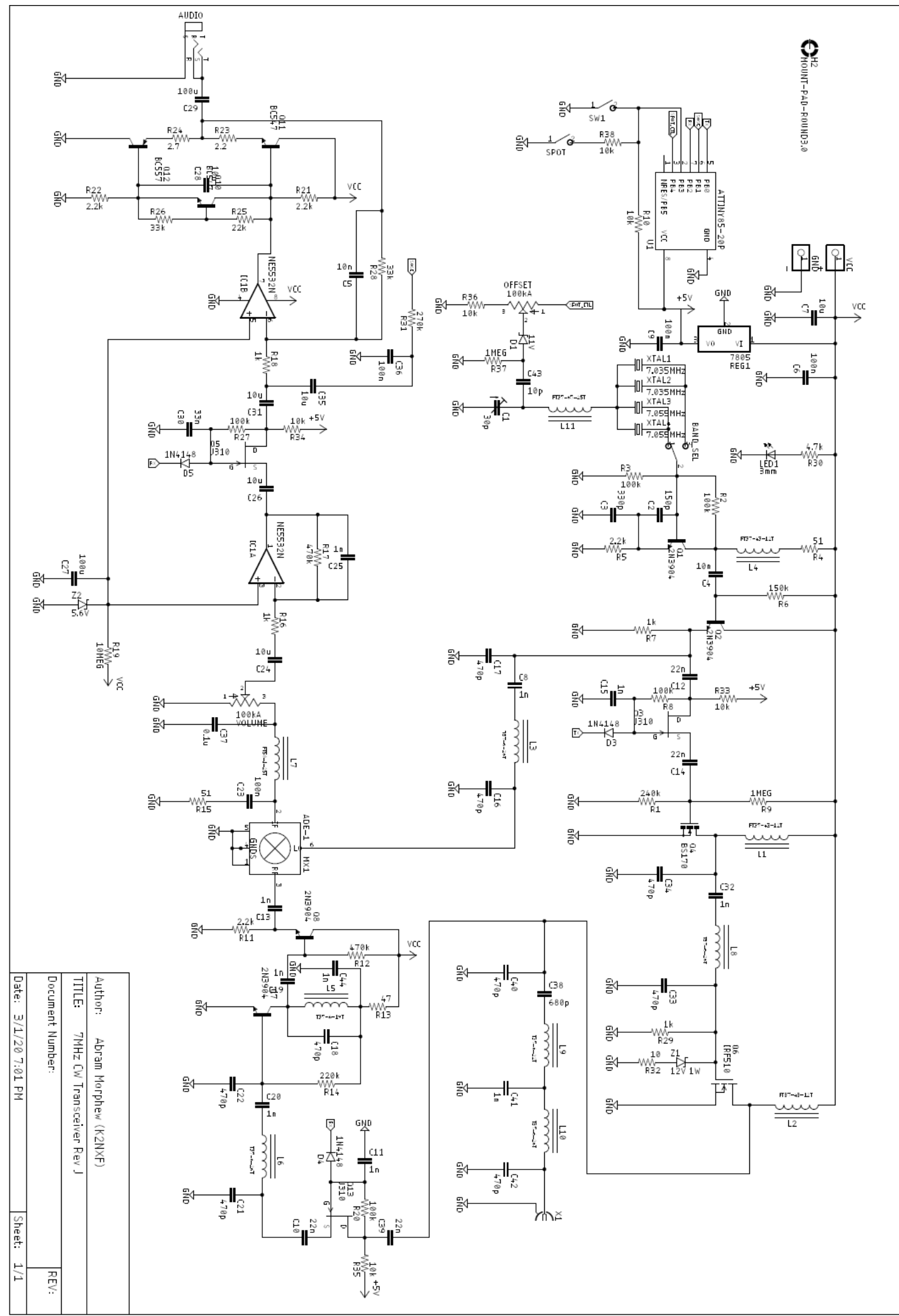

Figure 6.1: Full Transceiver Schematic 


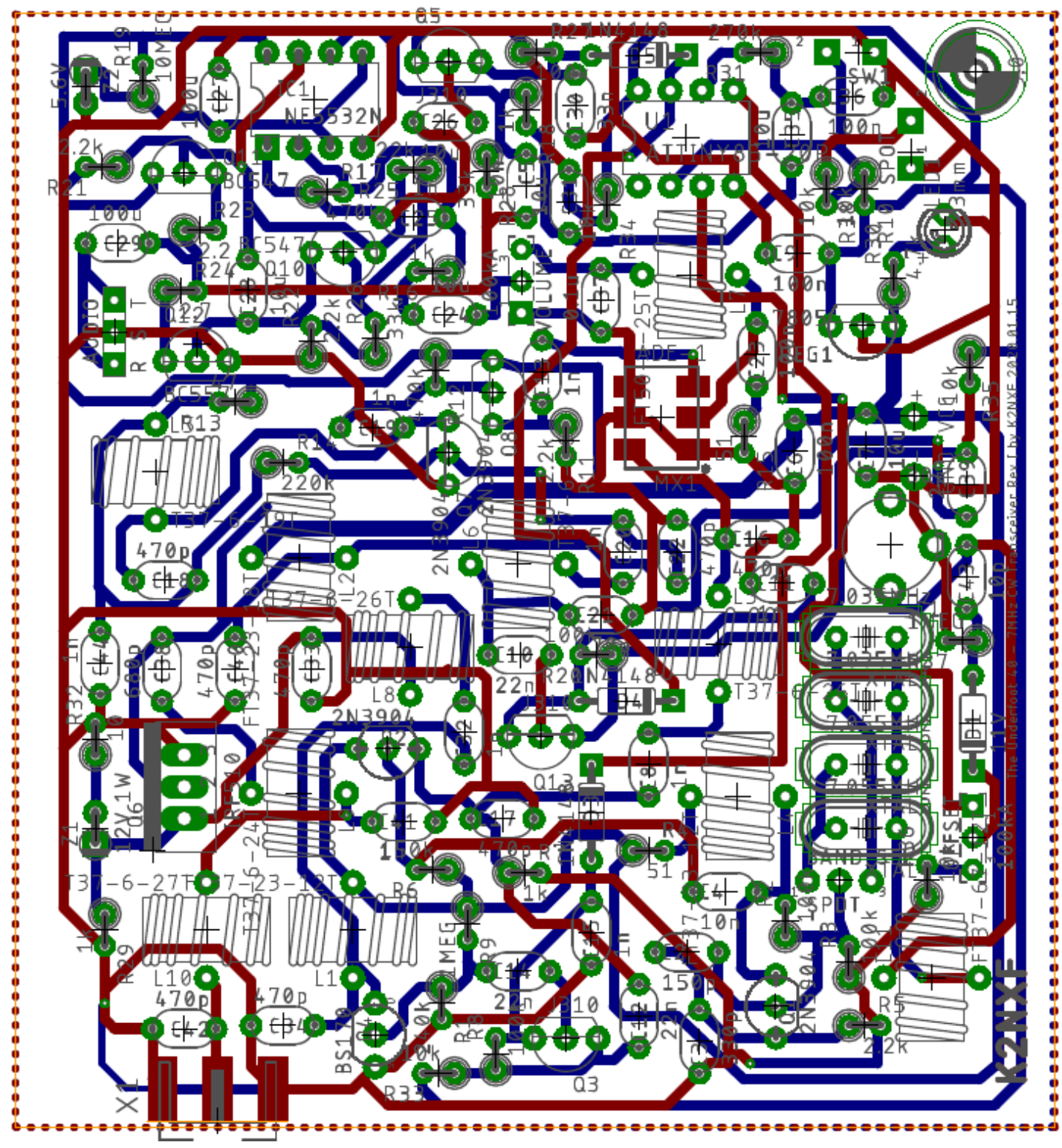

Figure 6.2: Full Transceiver Circuit Board Layout

the resonators is rather crowded. From frequency measurements of the LO, there must be some amount of board capacitance either from the traces or ground relief as the highest frequency possible from the LO was lower than the markings on the crystal by 4 to $5 \mathrm{kHz}$. Some care had to be taken in order to achieve a reasonable tuning range with a constant power output over the band.

Some adjustments also had to be made to the PA in order to reduce LO leakage on the output of the antenna port. The isolation of the JFET switch was such that a 


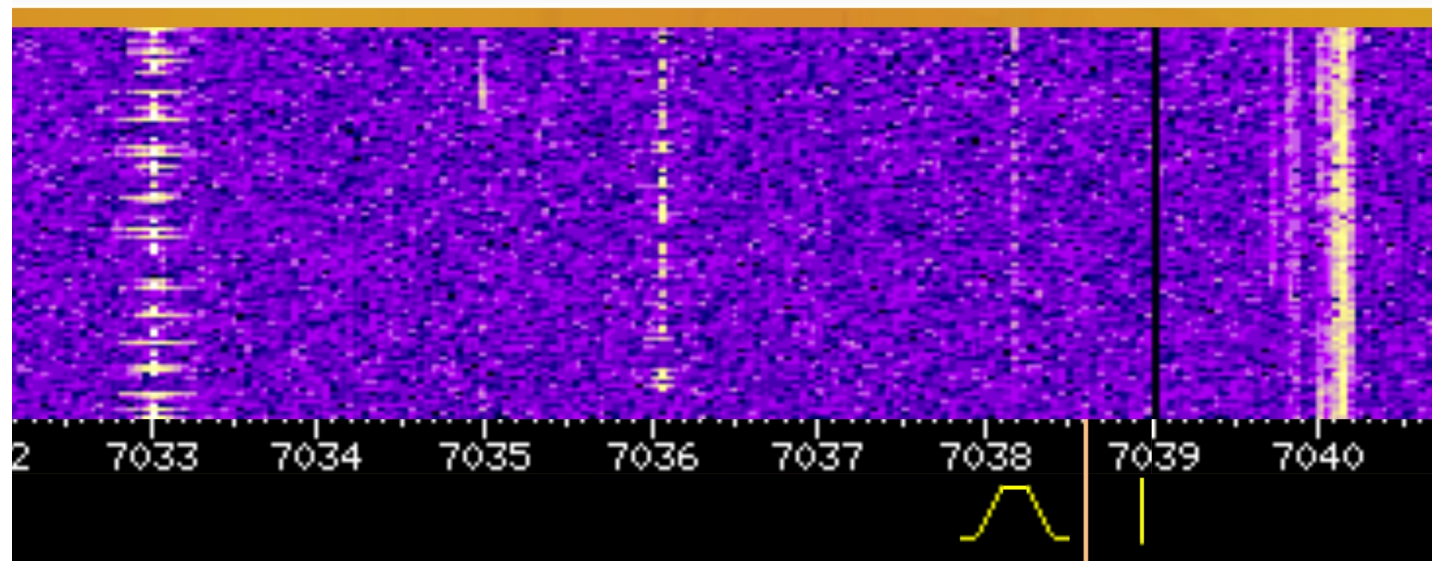

Figure 6.3: Transmitter Signal Received on a Software-Defined Radio in Corinne, Utah

small signal was appearing at the input to the BS170 driver stage. At the antenna, power levels of around $10 \mathrm{dBm}$ were originally observed. A small reduction in the bias voltage on the gate reduced LO leakage to a negligible level (around $-20 \mathrm{dBm}$ ) while also causing a $1.4 \mathrm{~dB}$ drop in overall output power. Ideally, a redesign of the bias control and an improvement in RF isolation of the JFET switch could aid in reducing the LO leakage, but the power amplifier did see a small boost in efficiency since the bias reduction slightly reduced the overall DC current. The power reduction also aided in some other issues where the high output signal during transmit could damage various unshielded components on the board.

\subsection{On-Air Test Results}

Testing the transceiver over the air proved to have its own challenges. Having done most on-air testing in a major metropolitan area, there are several sources of electromagnetic interference (EMI) resulting in heavy noise at times. Additionally, finding space to deploy an antenna for the $40 \mathrm{~m}$ band is also challenging. On-air tests were performed with either a wire dipole or long wire tuned for an approximate $50 \Omega$ match. 


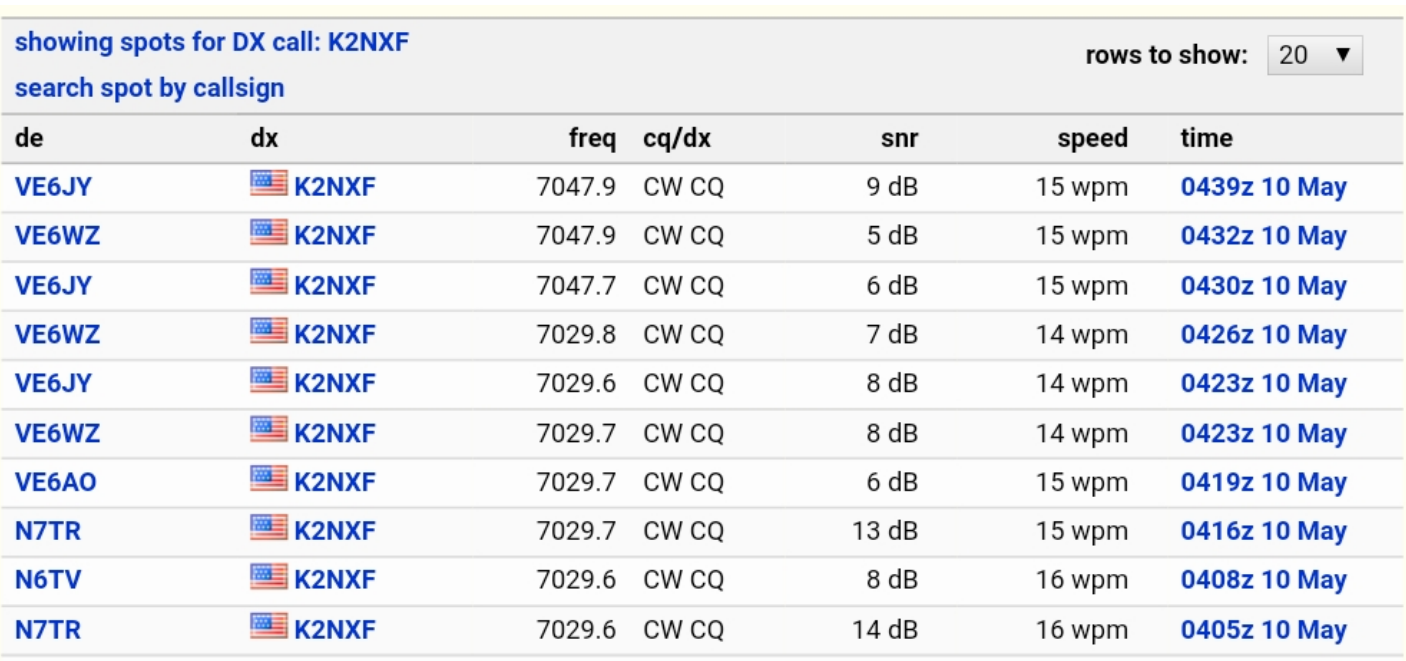

Figure 6.4: Stations Reporting Received Transmissions on the Reverse Beacon Network

The Reverse Beacon Network was extremely useful for observing the propagation of test transmissions, as shown in Figure 6.4. The furthest distance reported from Tucson, Arizona at a distance of 1103.5 miles from the source of the transmission. Additionally, SDR receivers in Utah and California provide access via the internet allowing for remote operation. Figure 6.3 shows a capture of the transceiver being received on 7.038 $\mathrm{MHz}$.

Reception tests involved recording the audio output signal with a digital recorder so that frequency analysis could be performed. In Figure 6.5, two sections of separate audio recordings can be seen as a logarithmic spectrogram. The spectrograph on the left side of the figure shows received audio without the various low-pass filters. The right side of the figure is an audio recording with the filters in place and a clear dropoff in signal strength around $3.6 \mathrm{kHz}$. This tracks well with the frequency response of the amplifier shown previously in 4.12 . Some stations can still be seen in the filtered audio capture, but the increased attenuation at higher frequencies adds to the overall selectivity of the receiver.

Both spectrographs in Figure 6.5 capture a number of distant stations. The strong 


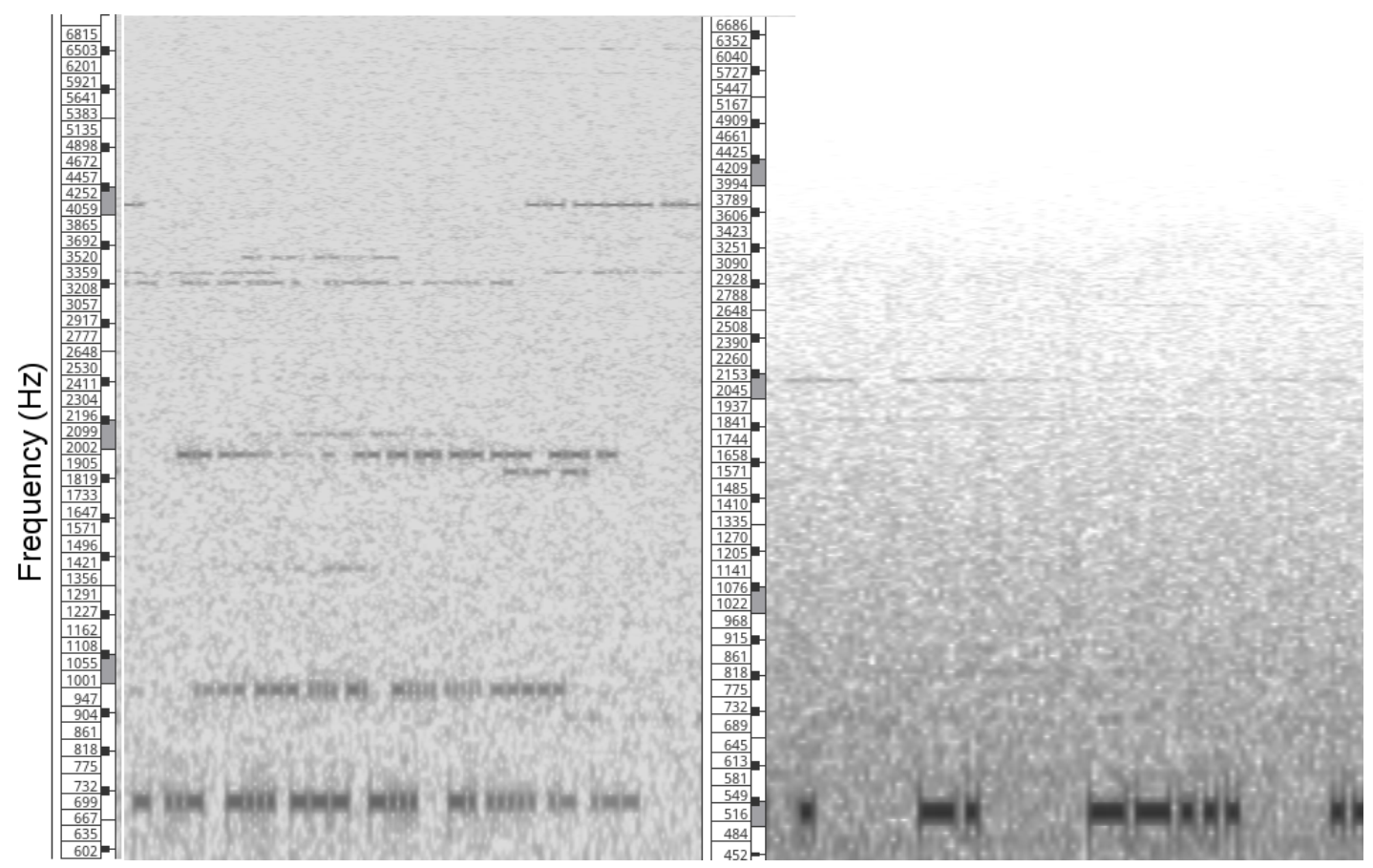

Figure 6.5: Logarithmic Audio Spectrograph with and without Multi-stage Filtering

signal around $690 \mathrm{~Hz}$ on the left side is station call sign N5AW in Burnet, Texas at a distance of 1660 miles from Portland, Oregon. On the right, station N7IV in Minot, North Dakota shows up around $549 \mathrm{~Hz}$ coming in from a distance of 1023 miles. When tested in remote areas, some stations were strong enough that the volume had to be adjusted to more comfortable levels despite less-than-optimal antenna deployment during test.

\subsection{Future Work}

Overall, the final build on PCB, as shown in Figure 6.6, performed very much in line with individual module tests. However, some minor component changes had to be made in order to improve general operability of the system. The value of the DC blocking capacitor at the input to the audio section was adjusted from $10 \mu F$ to 0.1 $\mu F$ to reduce oscillations. Due to leakage of RF back into the mixer during transmit, a strong impulse results as a loud pop on the audio output. When the system is first 


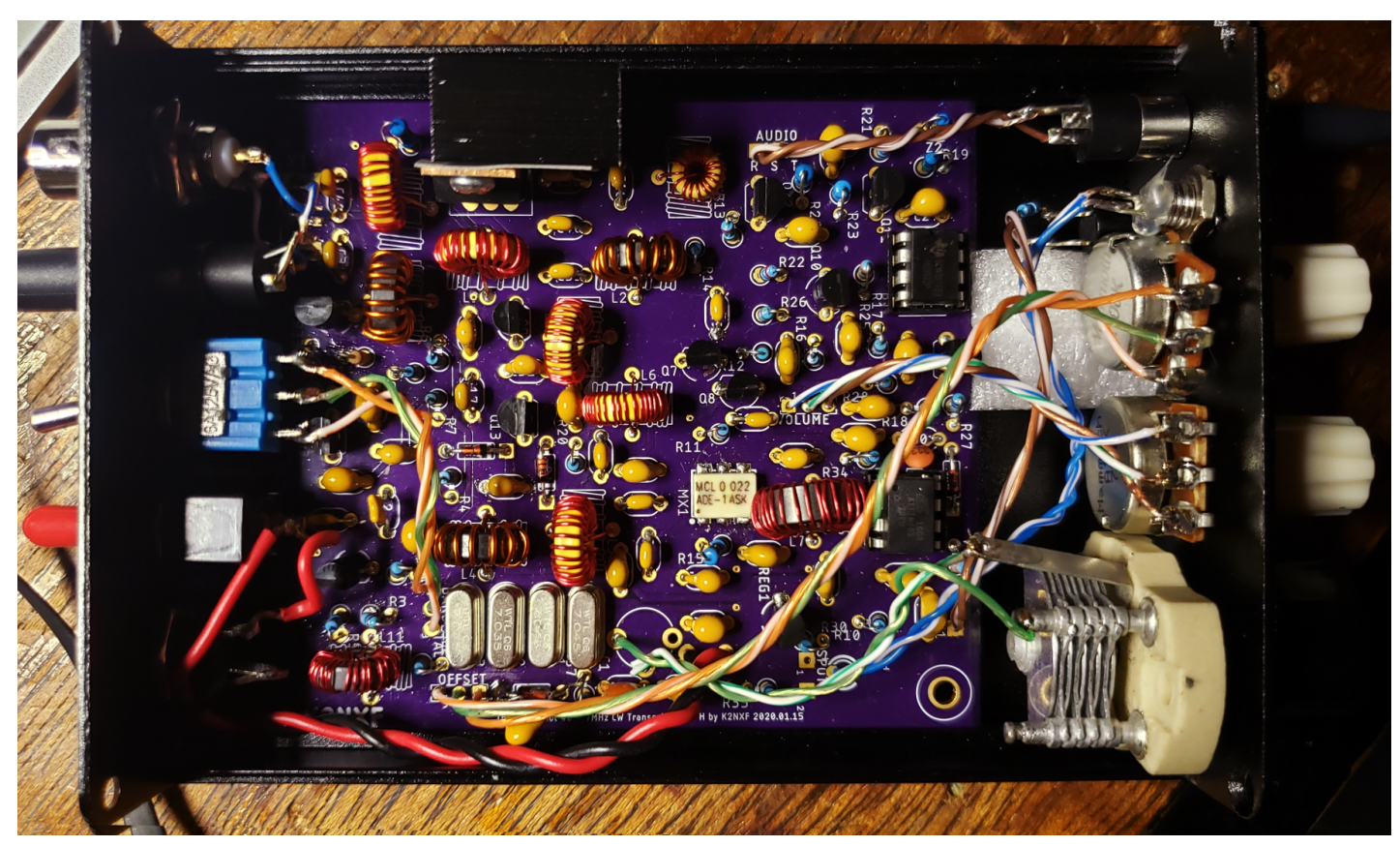

Figure 6.6: Assembled PCB Mounted in the Aluminum Enclosure

powered on or has not been keyed up in a while, the pop is still very apparent even with this modification, and a very low frequency oscillation can result in some situations. Additional design work could be done here to improve the interaction between the output of the mixer and input of the amplifier.

Additionally, switching between the two pairs of crystal resonators produced some odd results with respect to frequency tuning range. When comparing the pulling range of both sets, the pair of resonators with the lower frequency consistently had more than double the tuning range of the pair with the higher frequency. The lower frequency pair also seemed to have more stability issues towards the bottom end of the tuning range. In order to combat this problem, four crystals of the same frequency were used and connected in parallel. This provided more frequency stability and consistent power output from the LO while offering $15 \mathrm{kHz}$ of tuning range $(7.026 \mathrm{MHz}$ to 7.041 $\mathrm{MHz}$ ). While this is acceptable in terms of operation, having frequency agility across the entire CW portion of the $40 \mathrm{~m}$ band would be preferable. 


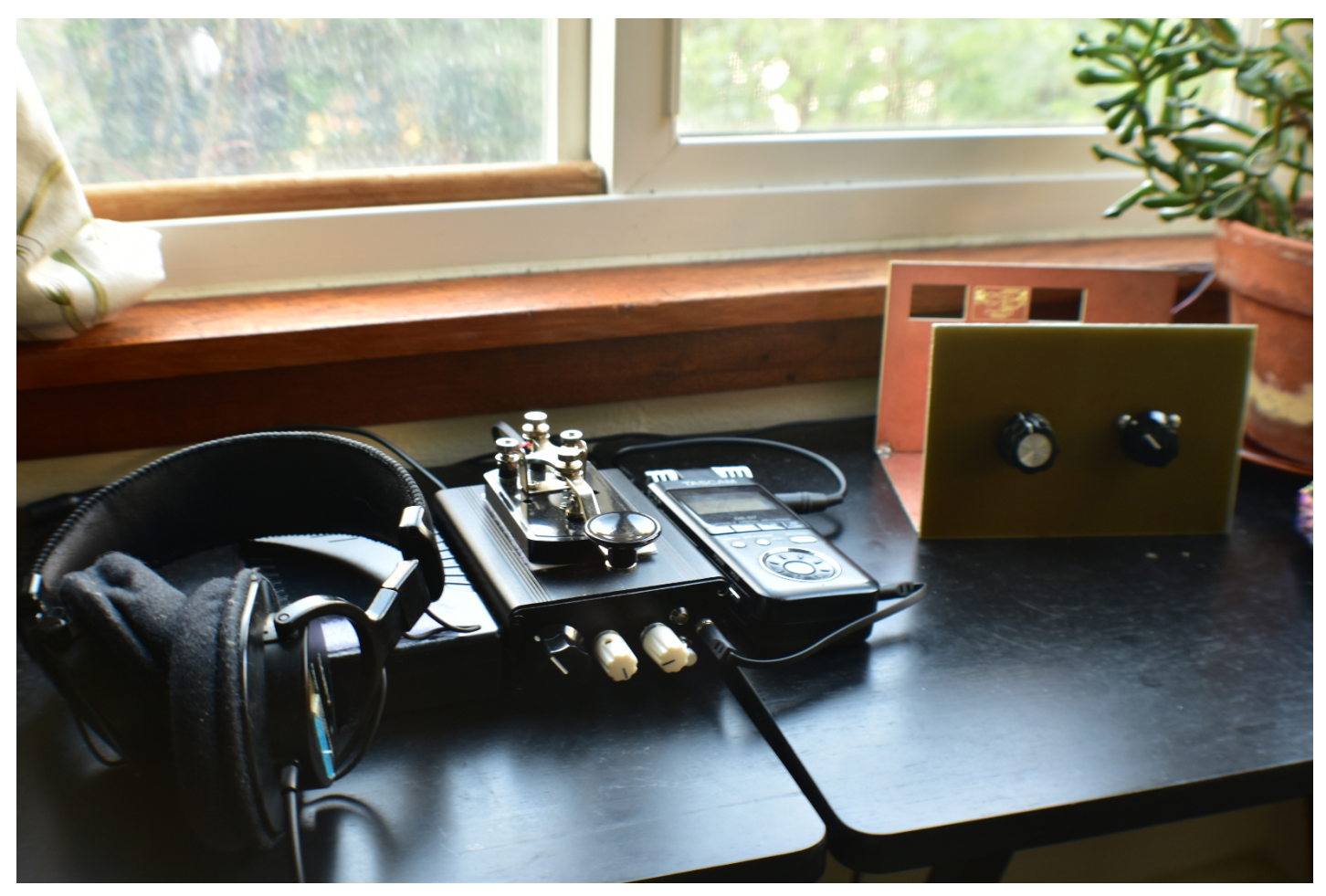

Figure 6.7: Completed Transceiver and On-air Test Station

\subsection{Conclusion}

Figure 6.7 shows the transceiver system configured for on-air testing. Many stations have been received under less-than-optimal conditions given the location in a major metropolitan area, and the transmitting signal was received by stations more than a thousand miles away. The addition of the MCU to a predominantly analog environment within the direct-conversion system did not result in any adverse effects and provided precision control to the TX, RX, and LO sections of the transceiver. With a $11.5 \mathrm{~V}$ supply to simulate the average voltage of a $12 \mathrm{~V}$ battery, the transceiver pulls $45 \mathrm{~mA}$ of current during receive and $520 \mathrm{~mA}$ of current during transmit. This level of current draw makes it ideal for portable use with AA alkaline batteries or rechargable batteries capable of producing a $12 \mathrm{~V}$ supply. 


\section{Appendix A}

\section{AVR Controller Program}

\section{A.1 Keying Control Code for AVR Microcontroller}

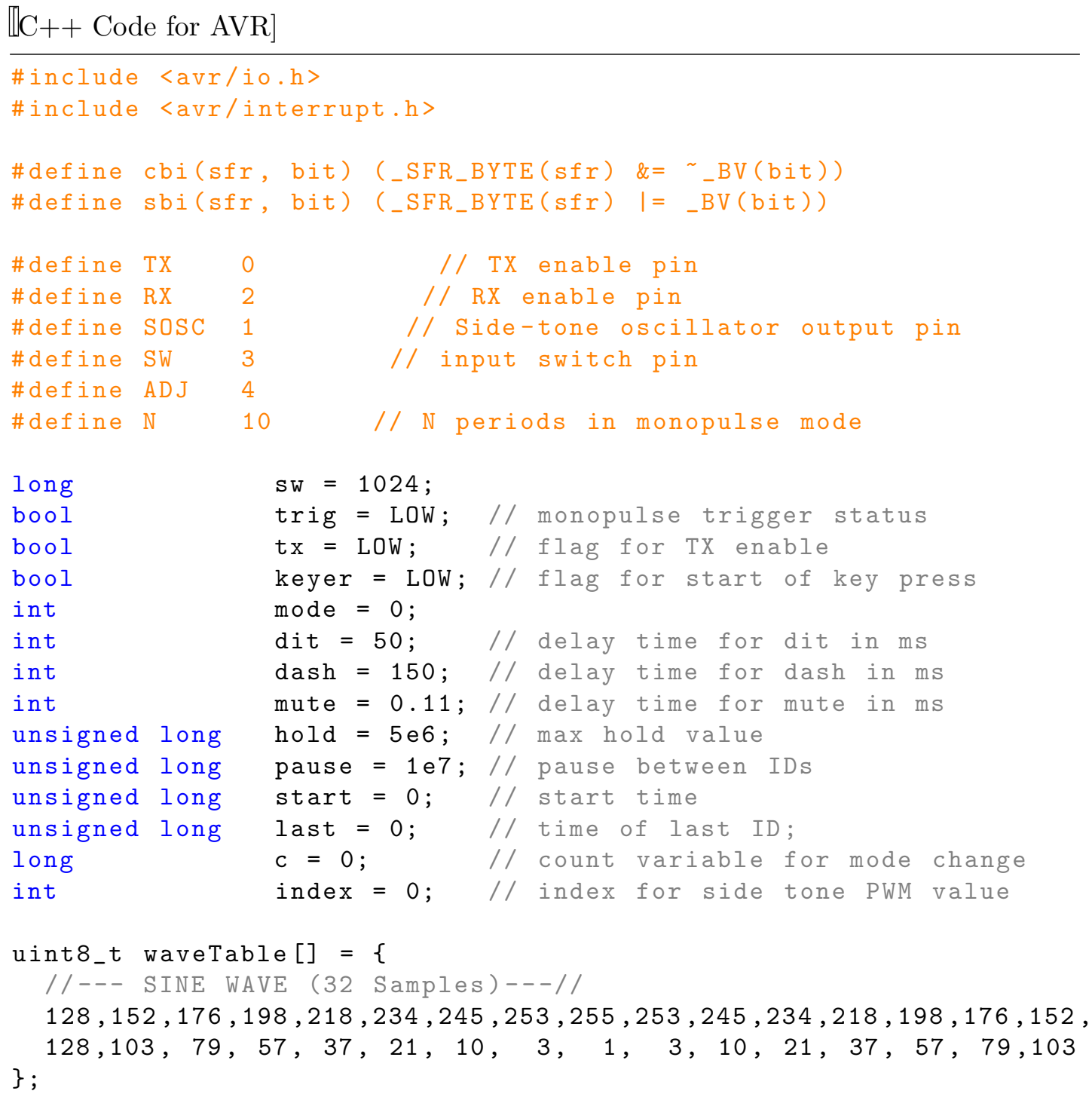




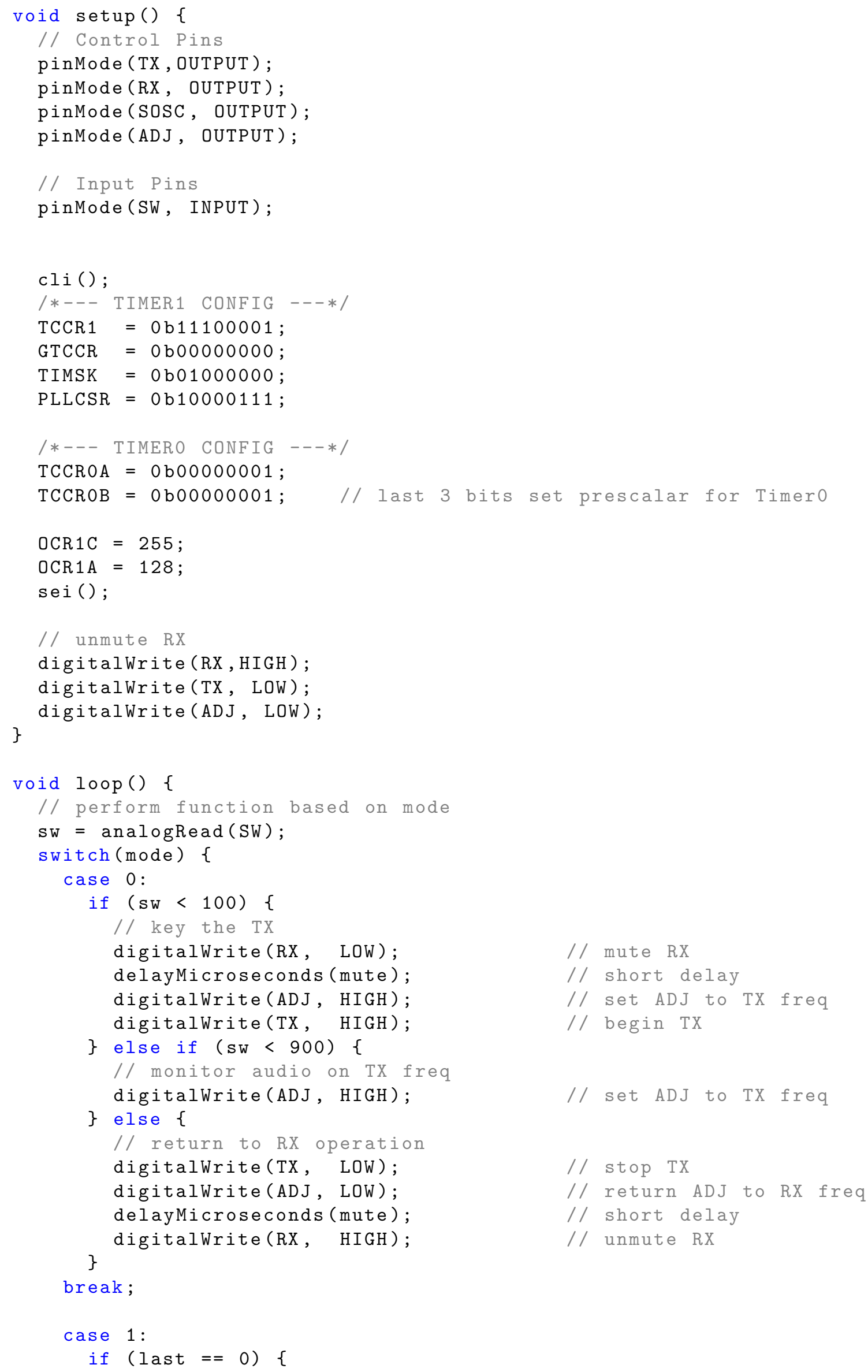




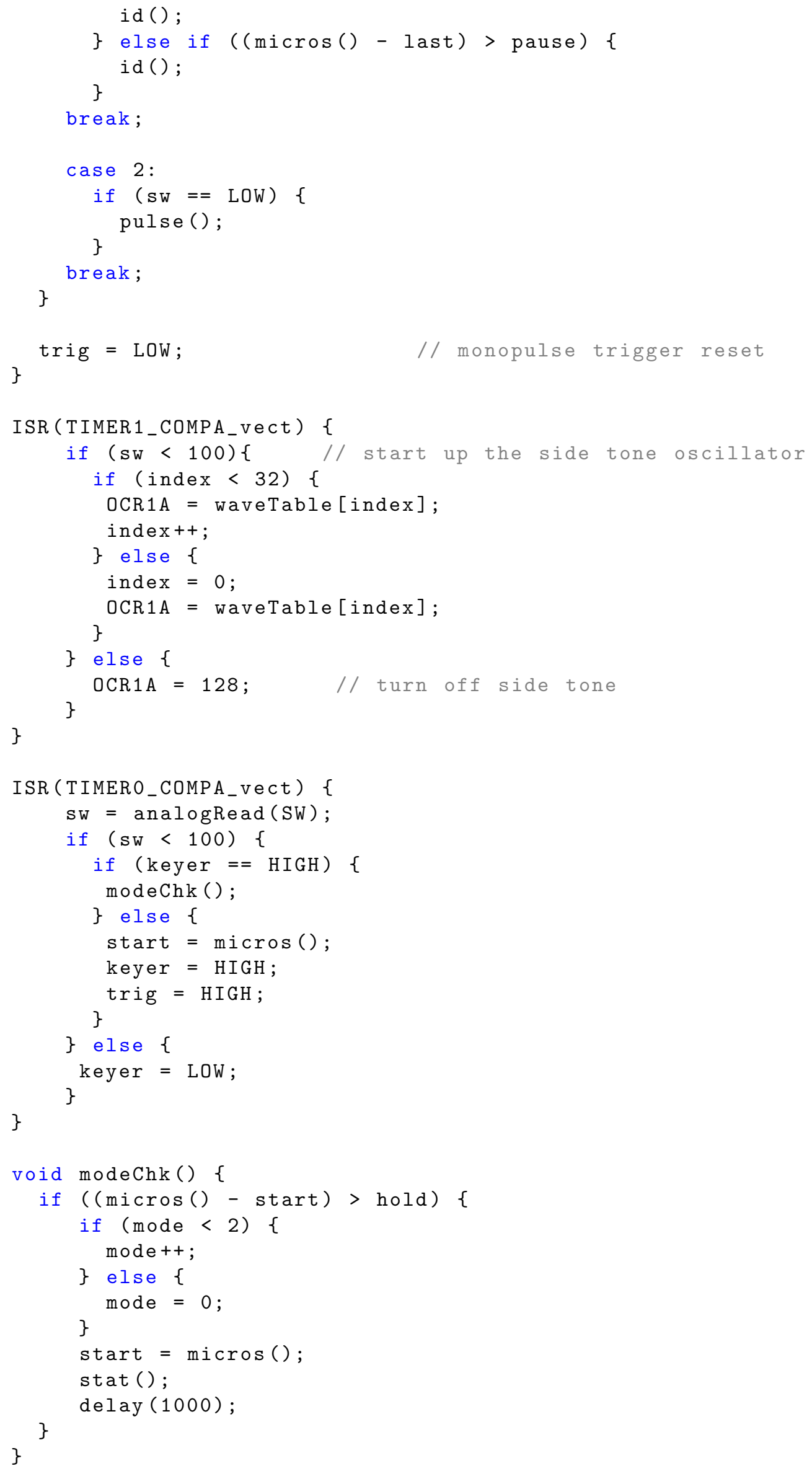




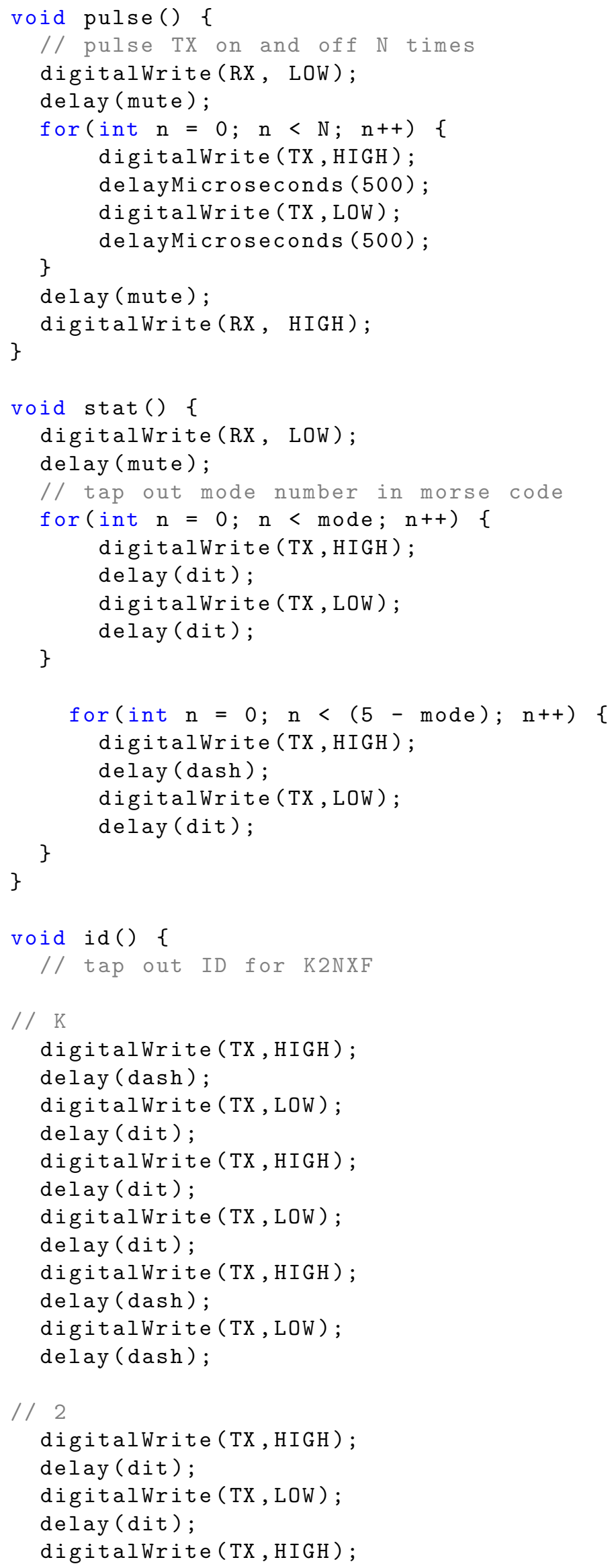




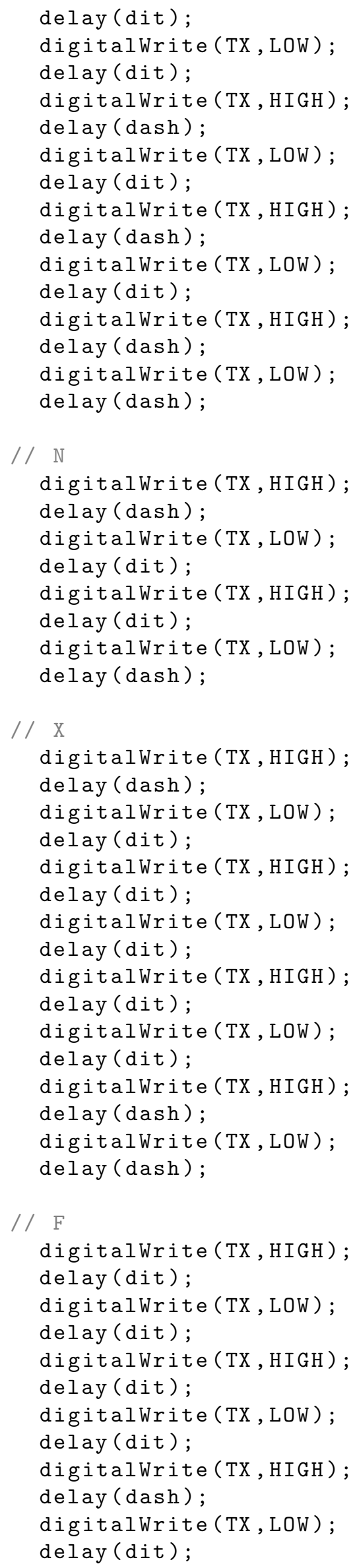




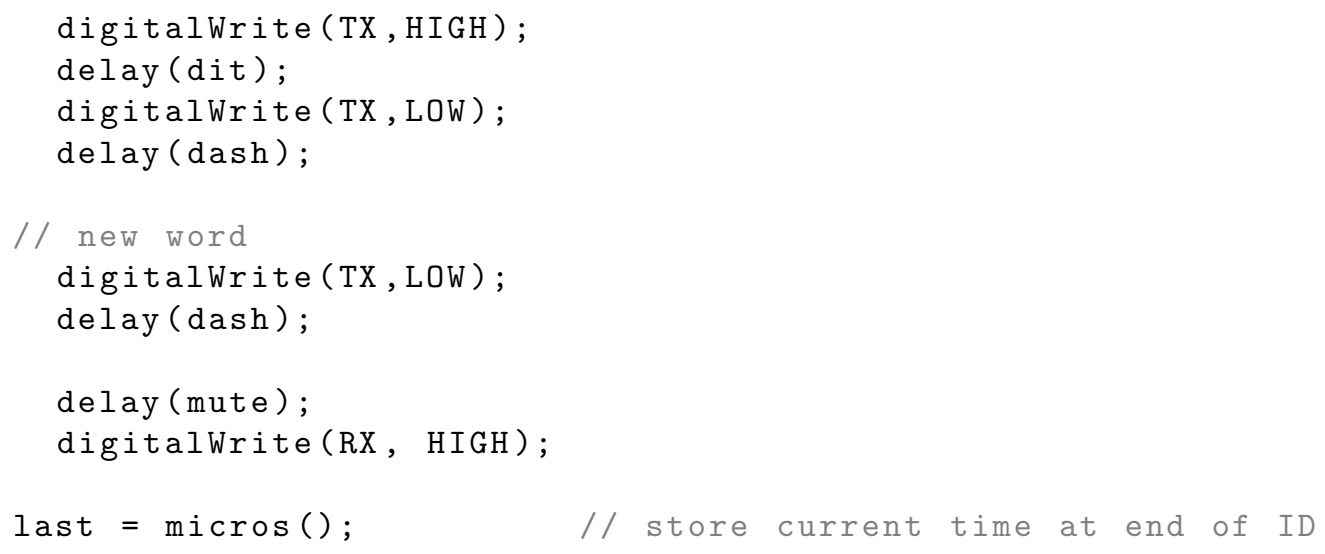




\section{Appendix B}

\section{Bill of Materials}

\section{B.1 List of Materials Used in the Final Transceiver}

\begin{tabular}{|c|c|c|c|c|}
\hline Qty & Value & Description & Price & Cost \\
\hline 1 & $78 \mathrm{~L} 05$ & $5 \mathrm{~V}$ Voltage Regulator & $\$ 0.67$ & $\$ 0.67$ \\
\hline 5 & $100 \mathrm{nF}$ & KEMET Ceramic Capacitor & $\$ 0.26$ & $\$ 1.30$ \\
\hline 2 & $100 \mathrm{uF}$ & KEMET Ceramic Capacitor & $\$ 0.26$ & $\$ 0.52$ \\
\hline 2 & $10 \mathrm{nF}$ & KEMET Ceramic Capacitor & $\$ 0.26$ & $\$ 0.52$ \\
\hline 1 & $10 \mathrm{pF}$ & KEMET Ceramic Capacitor & $\$ 0.26$ & $\$ 0.26$ \\
\hline 6 & $10 \mathrm{uF}$ & KEMET Ceramic Capacitor & $\$ 0.26$ & $\$ 1.56$ \\
\hline 1 & $150 \mathrm{pF}$ & KEMET Ceramic Capacitor & $\$ 0.26$ & $\$ 0.26$ \\
\hline 11 & $1 \mathrm{nF}$ & KEMET Ceramic Capacitor & $\$ 0.26$ & $\$ 2.86$ \\
\hline 4 & $22 \mathrm{nF}$ & KEMET Ceramic Capacitor & $\$ 0.26$ & $\$ 1.04$ \\
\hline 1 & $330 \mathrm{pF}$ & KEMET Ceramic Capacitor & $\$ 0.26$ & $\$ 0.26$ \\
\hline 1 & $33 \mathrm{nF}$ & KEMET Ceramic Capacitor & $\$ 0.26$ & $\$ 0.26$ \\
\hline 9 & $470 \mathrm{pF}$ & KEMET Ceramic Capacitor & $\$ 0.26$ & $\$ 2.34$ \\
\hline 1 & $680 \mathrm{pF}$ & KEMET Ceramic Capacitor & $\$ 0.26$ & $\$ 0.26$ \\
\hline 3 & $1 \mathrm{~N} 4148$ & Fast Switching Diode & $\$ 0.10$ & $\$ 0.30$ \\
\hline 1 & $12 \mathrm{~V}$ & Zener Diode (12 V, 1 Watt) & $\$ 0.24$ & $\$ 0.24$ \\
\hline 1 & $5.6 \mathrm{~V}$ & Zener Diode (5.6 V, 1 Watt) & $\$ 0.24$ & $\$ 0.24$ \\
\hline 1 & $11 \mathrm{~V}$ & Zener Diode (11 V, 1 Watt) & $\$ 0.26$ & $\$ 0.26$ \\
\hline 2 & 2N3904 & ON Semiconductor NPN Transistor & $\$ 0.20$ & $\$ 0.40$ \\
\hline 3 & J310 & InterFET N-Channel JFETs & $\$ 3.27$ & $\$ 9.81$ \\
\hline 1 & ADE-1 & Mini Circuits ADE-1+ Level 7 Mixer & $\$ 5.49$ & $\$ 5.49$ \\
\hline 1 & ATTINY85-20P & Atmel ATTiny85-20PU & $\$ 1.20$ & $\$ 1.20$ \\
\hline 4 & BC547 & ON Semiconductor NPN Transistor & $\$ 0.20$ & $\$ 0.80$ \\
\hline 1 & BC557 & ON Semiconductor PNP Transistor & $\$ 0.21$ & $\$ 0.21$ \\
\hline 1 & BS170 & Fairchild N-Channel Enhancement MOSFET & $\$ 0.45$ & $\$ 0.45$ \\
\hline 1 & $30 \mathrm{pF}$ & Trimming capacitor & $\$ 4.54$ & $\$ 4.54$ \\
\hline 2 & $7.055 \mathrm{MHz}$ & Crystal Resonator & $\$ 1.13$ & $\$ 2.26$ \\
\hline 1 & IRF510 & Vishay N-Channel Enhancement MOSFET & $\$ 0.88$ & $\$ 0.88$ \\
\hline 1 & $3 \mathrm{~mm}$ & LED & $\$ 0.10$ & $\$ 0.10$ \\
\hline 1 & NE5532N & Dual Operational Amplifier & $\$ 0.57$ & $\$ 0.57$ \\
\hline 2 & $100 \mathrm{kA}$ & 100k Logarithmic Potentiometers & $\$ 1.57$ & $\$ 3.14$ \\
\hline 1 & 10 & 1/8W Leaded Metal Film Resistor & $\$ 0.01$ & $\$ 0.01$ \\
\hline
\end{tabular}




\begin{tabular}{|l|c|c|c|c|}
\hline 5 & $100 \mathrm{k}$ & $1 / 8 \mathrm{~W}$ Leaded Metal Film Resistor & $\$ 0.01$ & $\$ 0.05$ \\
\hline 1 & $10 \mathrm{MEG}$ & $1 / 8 \mathrm{~W}$ Leaded Metal Film Resistor & $\$ 0.01$ & $\$ 0.01$ \\
\hline 6 & $10 \mathrm{k}$ & $1 / 8 \mathrm{~W}$ Leaded Metal Film Resistor & $\$ 0.01$ & $\$ 0.06$ \\
\hline 1 & $150 \mathrm{k}$ & $1 / 8 \mathrm{~W}$ Leaded Metal Film Resistor & $\$ 0.01$ & $\$ 0.01$ \\
\hline 2 & $1 \mathrm{MEG}$ & $1 / 8 \mathrm{~W}$ Leaded Metal Film Resistor & $\$ 0.01$ & $\$ 0.02$ \\
\hline 4 & $1 \mathrm{k}$ & $1 / 8 \mathrm{~W}$ Leaded Metal Film Resistor & $\$ 0.01$ & $\$ 0.04$ \\
\hline 1 & 2.2 & $1 / 8 \mathrm{~W}$ Leaded Metal Film Resistor & $\$ 0.01$ & $\$ 0.01$ \\
\hline 4 & $2.2 \mathrm{k}$ & $1 / 8 \mathrm{~W}$ Leaded Metal Film Resistor & $\$ 0.01$ & $\$ 0.04$ \\
\hline 1 & 2.7 & $1 / 8 \mathrm{~W}$ Leaded Metal Film Resistor & $\$ 0.01$ & $\$ 0.01$ \\
\hline 1 & $220 \mathrm{k}$ & $1 / 8 \mathrm{~W}$ Leaded Metal Film Resistor & $\$ 0.01$ & $\$ 0.01$ \\
\hline 1 & $22 \mathrm{k}$ & $1 / 8 \mathrm{~W}$ Leaded Metal Film Resistor & $\$ 0.01$ & $\$ 0.01$ \\
\hline 1 & $240 \mathrm{k}$ & $1 / 8 \mathrm{~W}$ Leaded Metal Film Resistor & $\$ 0.01$ & $\$ 0.01$ \\
\hline 1 & $270 \mathrm{k}$ & $1 / 8 \mathrm{~W}$ Leaded Metal Film Resistor & $\$ 0.01$ & $\$ 0.01$ \\
\hline 2 & $33 \mathrm{k}$ & $1 / 8 \mathrm{~W}$ Leaded Metal Film Resistor & $\$ 0.01$ & $\$ 0.02$ \\
\hline 1 & $4.7 \mathrm{k}$ & $1 / 8 \mathrm{~W}$ Leaded Metal Film Resistor & $\$ 0.01$ & $\$ 0.01$ \\
\hline 2 & $470 \mathrm{k}$ & $1 / 8 \mathrm{~W}$ Leaded Metal Film Resistor & $\$ 0.01$ & $\$ 0.02$ \\
\hline 2 & 51 & $1 / 8 \mathrm{~W}$ Leaded Metal Film Resistor & $\$ 0.01$ & $\$ 0.02$ \\
\hline 1 & $680 \mathrm{k}$ & $1 / 8 \mathrm{~W}$ Leaded Metal Film Resistor & $\$ 0.01$ & $\$ 0.01$ \\
\hline 1 & SMA & SMA 50 Ohm End Launch Jack Receptacle & $\$ 3.74$ & $\$ 3.74$ \\
\hline 2 & SPST & Push-button SPST Switch & $\$ 3.17$ & $\$ 6.34$ \\
\hline 1 & $3.5 \mathrm{~mm}$ & Stereo (TRS) 0.25 Inch Phone Jack & $\$ 1.10$ & $\$ 1.10$ \\
\hline 2 & FT37-23-12T & Inductor wound on a T37 Toroid & $\$ 0.49$ & $\$ 0.98$ \\
\hline 1 & FT37-23-18T & Inductor wound on a T37 Toroid & $\$ 0.49$ & $\$ 0.49$ \\
\hline 1 & FT37-67-12T & Inductor wound on a T37 Toroid & $\$ 0.49$ & $\$ 0.49$ \\
\hline 1 & FT50-J-25T & Inductor wound on a T50 Toroid & $\$ 3.64$ & $\$ 3.64$ \\
\hline 1 & T37-6-19T & Inductor wound on a T37 Toroid & $\$ 0.80$ & $\$ 0.80$ \\
\hline 1 & T37-6-24T & Inductor wound on a T37 Toroid & $\$ 0.80$ & $\$ 0.80$ \\
\hline 3 & T37-6-26T & Inductor wound on a T37 Toroid & $\$ 0.80$ & $\$ 2.40$ \\
\hline 1 & T37-6-27T & Inductor wound on a T37 Toroid & $\$ 0.80$ & $\$ 0.80$ \\
\hline 1 & PCB & Printed circuit board & $\$ 11.44$ & $\$ 11.44$ \\
\hline 1 & Enclosure & uxcell Aluminum Project Box 124x88x38mm & $\$ 13.27$ & $\$ 13.27$ \\
\hline & & & & \\
\hline
\end{tabular}

TABLE B.1: Bill of Materials

Total Cost: $\$ 89.67$ 


\section{Bibliography}

[1] Theremin, Leon. "US1661058: Method of and Apparatus for the Generation of Sounds." 5 Dec. 1925.

[2] Hayward, W. and Bingham, D. "Direct Conversion: A Neglected Technique." QST, Nov. 1968, pp.15-17.

[3] Krauss, Herbert L, et al. "Solid State Radio Engineering." New York, John Wiley \& Sons, 1980.

[4] K4SWL. "Elecraft Discontinues the K1 and KX1." Q R P e R, 31 Aug. 2017, qrper.com/2017/08/elecraft-discontinues-the-k1-and-kx1/. Accessed 12 May 2020 .

[5] "QCX+ 5W CW Transceiver Kit." Www.Qrp-Labs.Com, www.qrplabs.com/qcxp.html. Accessed 12 May 2020.

[6] Hayward, Wes. "Introduction to Radio Frequency Design." Newington, CT, The American Radio Relay League, Inc., 1994, Chap. 6

[7] Mini-Circuits, "Surface Mount Frequency Mixer." ADE-1/ADE-1+ Datasheet Rev F.

[8] American Radio Relay League, and Bill Troetschel. "The ARRL UHF/Microwave Experimenters Manual: Antennas, Components and Design." 4th ed., Newington, CT, ARRL, 1997, pp. 647.

[9] Hayward, Wes, et al. "Experimental Methods in RF Design." Newington, CT, American Radio Relay League, 2009.

[10] Love, Janine Sullivan. "RF Front-End: World Class Designs." Burlington, MA, Newnes, 2009.

[11] Ashworth, Dennis, et al. "The ARRL Handbook for Radio Amateurs." 73rd ed., Newington, CT, American Radio Relay League, 1995.

[12] Frerking, M E. "Crystal Oscillator Design and Temperature Compensation." New York, N.Y., Van Nostrand Reinhold, 1978.

[13] Carr, Joseph. "RF Components and Circuits." Woburn, MA, Newnes, 2002, pp. 126145. 
[14] Davis, W. Alan, and Krishna Agarwal. "Radio Frequency Circuit Design." Hoboken, NJ, John Wiley \& Sons, Inc., 2001.

[15] Matko, Vojko, and Riko afari. "Major Improvements of Quartz Crystal Pulling Sensitivity and Linearity Using Series Reactance." Sensors, vol. 9, no. 10, 19 Oct. 2009, pp. 82638270, 10.3390/s91008263. Accessed 12 Oct. 2019 .

[16] Bare, Daniel, and John Clements. "Wide Range Crystal Pulling For Homebrew Radio." 2017. http://kc9on.com/wpcontent/uploads/2017/01/SVXOCrystalPullArticle.pdf

[17] Gray, Paul, et al. "Analysis and Design of Analog Integrated Circuits." 5th ed., New York, NY, Wiley, 2009, pp. 21-22.

[18] Lewallen, Roy. "An Optimized QRP Transceiver." QST, Aug. 1980, pp. 1419.

[19] Nilsson, James W, and Susan A Riedel. "Electric Circuits". 10th ed., New York, NY, Pearson, 2015, pp. 176180.

[20] Neaman, David. "Microelectronics: Circuit Analysis And Design." 4th ed., Mcgraw-Hill Science Engineering, 2007, pp. 10621074.

[21] Hayward, Wes, and Doug Demaw. Solid State Design for the Radio Amateur. Newington, CT, American Radio Relay League, 1986.

[22] "FCC Online Table of Frequency Allocations". Federal Communication Commission, 2016. https://transition.fcc.gov/oet/spectrum/table/fcctable.pdf

[23] Bartelink, E.H.B., et al. "A Flat-Response Single-Tuned I. F. Amplifier." Proceedings of the IRE, vol. 36, no. 4, Apr. 1948, pp. 474478, 10.1109/jrproc.1948.229649. Accessed 29 Jan. 2020.

[24] Pederson, Donald, and Kartikeya Mayaram. "Analog Integrated Circuits for Communication: Principles, Simulation and Design." 2nd ed., New York; London, Springer, 2008, pp. 247280.

[25] Self, Douglas. "Audio Power Amplifier Design." Oxford, UK, Elsevier Ltd., 2009.

[26] Campbell, Rick. "High-Performance Direct Conversion Receivers." QST, Aug. 1992, pp. 20-28.

[27] Cripps, Steve. "RF Power Amplifiers for Wireless Communications." 2nd ed., Norwood, MA, Artech House, 2006.

[28] Fairchild Semiconductor Corporation, "N-Channel Enhancement Mode Field Effect Transistor." BS170/MMBF170 Datasheet Rev E2, 2010. 
[29] Sweet, Allen. "Designing Bipolar Transistor Radio Frequency Integrated Circuits." Boston, MA, Artech House, 2008.

[30] Cripps, Steve. "Advanced Techniques in RF Power Amplifier Design." Boston, MA, Artech House, 2002.

[31] Rhea, Randall. "Discrete Oscillator Design: Linear, Nonlinear, Transient, and Noise Domains." Boston, MA, Artech House, 2010.

[32] Rogers, John, and Calvin Plett. "Radio Frequency Integrated Circuit Design." Norwood, MA, Artech House, 2003.

[33] Vishay Siliconix, "Power MOSFET." IRF510/SiHF510 Datasheet S15-2693Rev C., 2015.

[34] Fairchild Semiconductor Corporation, "N-Channel RF Amplifier." J309/J310/MMBFJ309/MMBFJ309 Datasheet, 1997.

[35] Atmel Corporation, "Atmel 8-bit AVR Microcontroller with 2/4/8K Bytes In-System Programmable Flash." ATTiny25/ATTiny45/ATTiny85 Datasheet Rev 2586QAVR08/2013, Aug 2013.

[36] "Understanding Earphone / Headphone Specifications." Shure: Service and Repair, 29 Jan. 2019, www.shure.com/en-GB/support/find-ananswer/understanding-earphone-headphone-specifications. Accessed 24 May. 2019 .

[37] "Part 97 - Amateur Radio Service." Federal Communications Commision, Mar. 5 2018, www.arrl.org/part-97-text. Accessed 7 Apr. 2019. 